\title{
FORMES DE TORSION ANALYTIQUE ET FAMILLES DE SUBMERSIONS II*
}

\author{
XIAONAN MA ${ }^{\dagger}$
}

\begin{abstract}
In this paper, we continue the work [Ma1]. We finally prove the functoriality of the analytic torsion forms with respect to the composition of two submersions. This result extends to a relative situation a result by Berthomieu-Bismut, proved in the case where the second projection is on a point.
\end{abstract}

0. Introduction. Le but de ce travail est de démontrer la fonctorialité des formes de torsion analytique relativement à la composition de deux submersions. Cet article est la deuxième partie de ce travail.

Soient $W, V, S$ des variétés complexes. Soient $\pi_{1}: W \rightarrow V, \pi_{2}: V \rightarrow S$ des submersions holomorphes de fibres compactes $X, Y$. Alors $\pi_{3}=\pi_{2} \circ \pi_{1}: W \rightarrow S$ est une submersion holomorphe de fibre compacte $Z$. Soit $\xi$ un fibré vectoriel holomorphe sur $W$.

Soient $R^{\bullet} \pi_{1 *} \xi, R^{\bullet} \pi_{3 *} \xi, R^{\bullet} \pi_{2 *} R^{\bullet} \pi_{1 *} \xi$ les images directes de $\xi, \xi, R^{\bullet} \pi_{1 *} \xi$. On suppose que les $R^{\bullet} \pi_{1 *} \xi, R^{\bullet} \pi_{3 *} \xi, R^{\bullet} \pi_{2 *} R^{\bullet} \pi_{1 *} \xi$ sont localement libres. Soit $H\left(Z, \xi_{\mid Z}\right)$, $H\left(X, \xi_{\mid X}\right)$ les cohomologies de $\xi_{\mid Z}, \xi_{\mid X}$. Alors $H\left(Z, \xi_{\mid Z}\right)$ est un fibré holomorphe Zgradué sur $S$. Plus exactement $R^{\bullet} \pi_{3 *} \xi=H\left(Z, \xi_{\mid Z}\right)$. De même $R^{\bullet} \pi_{1 *} \xi=H\left(X, \xi_{\mid X}\right)$.

Soit $\omega^{V}$ (resp. $\left.\omega^{W}\right)$ une $(1,1)$ forme réelle, fermée sur $V$ ( resp. $W$ ), dont la restriction à chaque fibre $Y$ (resp. $Z$ ) définit une métrique hermitienne $g^{T Y}$ (resp. $g^{T Z}$ ) sur le fibré tangent relatif $T Y$ (resp. $T Z$ ). Soit $g^{T X}$ la métrique hermitienne sur $T X$ induite par $\omega^{W}$. Soit $h^{\xi}$ une métrique hermitienne sur $\xi$.

Soient $h^{H\left(X, \xi_{\mid X}\right)}=h^{R \pi_{1 *} \xi}, h^{H\left(Z, \xi_{\mid Z}\right)}$ et $h^{R \pi_{2 *} R \pi_{1 *} \xi}$ les métriques $L_{2}$ sur $H\left(X, \xi_{\mid X}\right)$, $H\left(Z, \xi_{\mid Z}\right)$ et $R^{\bullet} \pi_{2 *} R^{\bullet} \pi_{1 *} \xi$ correspondant à $g^{T X}, h^{\xi} ; g^{T Z}, h^{\xi}$ et $g^{T Y}, h^{R \pi_{1 *} \xi}$.

Soient $T_{1}\left(\omega^{W}, h^{\xi}\right), T_{2}\left(\omega^{V}, h^{R \pi_{1 *} \xi}\right), T_{3}\left(\omega^{W}, h^{\xi}\right)$ les formes de torsion analytique construites dans Bismut-Köhler [BKö] sur $V, S, S$ associées à $\left(\pi_{1}, \omega^{W}, h^{\xi}\right)$, $\left(\pi_{2}, \omega^{V}, h^{R \pi_{1 *} \xi}\right),\left(\pi_{3}, \omega^{W}, h^{\xi}\right)$. Les formes $T_{1}$ vérifient l'équation

$$
\frac{\bar{\partial} \partial}{2 \pi i} T_{1}\left(\omega^{W}, h^{\xi}\right)=\operatorname{ch}\left(R^{\bullet} \pi_{1 *} \xi, h^{R \pi_{1 *} \xi}\right)-\int_{X} \operatorname{Td}\left(T X, g^{T X}\right) \operatorname{ch}\left(\xi, h^{\xi}\right) .
$$

Soit $\widetilde{\mathrm{Td}}\left(T Z, T Y, g^{T Z}, g^{T Y}\right) \in P^{W} / P^{W, 0}$ la classe de Bott-Chern définie en [Ma1, (3.2)], telle que

$$
\begin{aligned}
\frac{\bar{\partial} \partial}{2 \pi i} \widetilde{\mathrm{Td}}\left(T Z, T Y, g^{T Z}, g^{T Y}\right)=\operatorname{Td}\left(T Z, g^{T Z}\right) \\
-\pi_{1}^{*}\left(\operatorname{Td}\left(T Y, g^{T Y}\right)\right) \operatorname{Td}\left(T X, g^{T X}\right) .
\end{aligned}
$$

Soit $P^{S}$ l'espace vectoriel de formes réelles sur $S$ qui sont la somme de formes $\mathcal{C}^{\infty}$ de type $(p, p)$. Soit $P^{S, 0}$ l'espace des $\alpha \in P^{S}$, qui s'écrivent sous la forme $\alpha=\partial \beta+\bar{\partial} \gamma$, où $\beta, \gamma$ sont des formes $\mathcal{C}^{\infty}$ sur $S$.

Pour $s \in S$, il existe une suite spectrale de Leray $\left(E_{r, s}, d_{r, s}\right)(r \geq 2)$ [Grot] telle que $E_{2}=R^{\bullet} \pi_{2 *} R^{\bullet} \pi_{1 *} \xi$. Soit $h^{E_{2}}$ la métrique sur $E_{2}$ induite par $h^{R \pi_{2 *} R \pi_{1 *} \xi}$.

\footnotetext{
${ }^{*}$ Received January 19, 1999; accepted for publication October 8, 1999.

${ }^{\dagger}$ Humboldt-Universitat zu Berlin, Institut für Mathematik, Rudower Chaussee 25, 12489 Berlin, Germany (xiaonan@mathematik.hu-berlin.de).
} 
On suppose que le rang des $E_{r}(r \geq 2)$ est localement constant sur $S$. On définit une classe de Bott-Chern $\widetilde{\operatorname{ch}}\left(E_{2}, H\left(Z, \xi_{\mid Z}\right), h^{E_{2}}, h^{H\left(Z, \xi_{\mid Z}\right)}\right) \in P^{S} / P^{S, 0}$ par la formule

$$
\begin{array}{r}
\tilde{\operatorname{ch}}\left(E_{2}, H\left(Z, \xi_{\mid Z}\right), h^{E_{2}}, h^{H\left(Z, \xi_{\mid Z}\right)}\right)=\Sigma_{r=2}^{\infty} \tilde{\operatorname{ch}}\left(E_{r}, E_{r+1}, h^{E_{r}}, h^{E_{r+1}}\right) \\
-\tilde{\operatorname{ch}}\left(H\left(Z, \xi_{\mid Z}\right), E_{\infty}, h^{H\left(Z, \xi_{\mid Z}\right)}, h^{E_{\infty}}\right) .
\end{array}
$$

où les $h^{E_{r}}$ sont des métriques hermitiennes sur $E_{r}$, et les $\tilde{\operatorname{ch}}\left(E_{r}, E_{r+1}, h^{E_{r}}, h^{E_{r+1}}\right)$, $\widetilde{c h}\left(H\left(Z, \xi_{\mid Z}\right), E_{\infty}, h^{H\left(Z, \xi_{\mid Z}\right)}, h^{E_{\infty}}\right) \in P^{S} / P^{S, 0}$ sont des classes de Bott-Chern au sens de [BGS1]. Alors

$$
\begin{aligned}
\frac{\bar{\partial} \partial}{2 \pi i} & \widetilde{\operatorname{h}}\left(E_{2}, H\left(Z, \xi_{\mid Z}\right), h^{E_{2}}, h^{H\left(Z, \xi_{\mid Z}\right)}\right) \\
& =\operatorname{ch}\left(H\left(Z, \xi_{\mid Z}\right), h^{H\left(Z, \xi_{\mid Z}\right)}\right)-\operatorname{ch}\left(E_{2}, h^{E_{2}}\right) .
\end{aligned}
$$

Dans [Ma1], on a donné une construction algébrique de la classe $\tilde{c h}\left(E_{2}, H\left(Z, \xi_{\mid Z}\right), h^{E_{2}}\right.$, $\left.h^{H\left(Z, \xi_{\mid Z}\right)}\right)$ dans les cas suivants: i) $\xi$ est $\pi_{1 *}$ et $\pi_{3 *}$ acyclique; ii) Les $\pi_{1}$ et $V$ sont projectives. En particulier, dans [Ma1], les $E_{r},(3 \leq r \leq \infty)$ ne sont pas nécessairement de rang constant. Toutefois quand les hypothèses de [Ma1] et les hypothèses du présent article sont vérifiées simultanément, on montre que ces deux constructions de $\widetilde{\operatorname{ch}}\left(E_{2}, H\left(Z, \xi_{\mid Z}\right), h^{E_{2}}, h^{H\left(Z, \xi_{\mid Z}\right)}\right)$ sont équivalentes.

Le but de cet article est de montrer le Théorème suivant.

THÉORÈME 0.1. On a l'identité

$$
\begin{aligned}
& T_{3}\left(\omega^{W}, h^{\xi}\right)-T_{2}\left(\omega^{V}, h^{R \pi_{1 *} \xi}\right)-\int_{Y} \operatorname{Td}\left(T Y, g^{T Y}\right) T_{1}\left(\omega^{W}, h^{\xi}\right) \\
& \quad+\int_{Z} \widetilde{\operatorname{Td}}\left(T Z, T Y, g^{T Z}, g^{T Y}\right) \operatorname{ch}\left(\xi, h^{\xi}\right) \\
& \quad-\operatorname{ch}\left(E_{2}, H\left(Z, \xi_{\mid Z}\right), h^{E_{2}}, h^{H\left(Z, \xi_{\mid Z}\right)}\right)=0 \text { dans } P^{S} / P^{S, 0} .
\end{aligned}
$$

L'énoncé du Théorème 0.1 est formellement identique à l'énoncé du résultat principal de [Ma1]. Cette identité formelle implique a posteriori que les définitions de $\widetilde{c h}\left(E_{2}, H\left(Z, \xi_{\mid Z}\right), h^{E_{2}}, h^{H\left(Z, \xi_{\mid Z}\right)}\right)$ dans [Ma1] et dans cet article sont équivalentes.

Cet article est organisé de la façon suivante. À la Section 1, on montre un analogue du Théorème 0.1 en dimension finie. La Section 1 nous servira de modèle pour la preuve du Théorème 0.1 , qui est donnée à la Section 2 . De plus, elle nous permettra de montrer directement que les définitions de $\widetilde{c h}\left(E_{2}, H\left(Z, \xi_{\mid Z}\right), h^{E_{2}}, h^{H\left(Z, \xi_{\mid Z}\right)}\right)$ données dans [Ma1] et dans notre article sont compatibles. À la Section 2, on montre d'abord que le complexe de Dolbeault muni d'une filtration convenable calcule la suite spectrale de Leray au sens de Grothendieck. On montre enfin la fonctorialité des formes de torsion analytique, le Théorème 0.1 .

On se réfère à l'introduction de [Ma1] pour une déscription générale de nos résultats. On utilise aussi les notations de [Ma1, $\S 3,4,5,10(\mathrm{a})]$.

Dans tout l'article, si $\mathcal{A}$ est une algèbre $\mathbf{Z}_{2}$-graduée, si $A, B \in \mathcal{A},[A, B]$ désigne le supercommutateur de $A$ et de $B$.

1. Class de Bott-Chern d'un bicomplexe. Soit $(\mathcal{E}, d, v)$ un bicomplexe de fibrés vectoriels holomorphes sur une variété complexe $S$. Soit $\left(\mathcal{E}_{r}, d_{r}\right)$ la suite spectrale associée à la filtration par $v$. On suppose que les $\mathcal{E}_{r}$ sont des fibrés vectoriels holomorphes sur $S$. Dans cette Section, on établit une relation entre la class de BottChern du complexe total $(\mathcal{E}, d+v)$ et les classes de Bott-Chern de $\left(\mathcal{E}_{r}, d_{r}\right)$. Le résultat principal de cette Section est énoncé au Théorème 1.2. 
Une stratégie possible pour démontrer le Théorème 1.2 serait d'utiliser la déformation sur $\mathbf{P}^{1}$ [BGS1, §1(f)] et [BGS4, §4]. On obtiendrait un bicomplexe de fibrés holomorphes sur $S \times \mathbf{P}^{1}$, qui coïncide avec le bicomplexe donné sur $S \times\{0\}$, qui a tous les propriétés de ce bicomplexe sur $S \times \mathbf{P}^{1} \backslash\{\infty\}$, mais qui est tel que sur $S \times\{\infty\}$, la suite spectrale dégénère en $E_{2}$. Mais, en général, les $\left(\mathcal{E}_{r}^{\prime}, d_{r}^{\prime}\right)$ relativement à ce nouveau bicomplexe ne sont pas localement libres, ce qui nous interdit d'utiliser cette méthode. On est donc amené à utiliser une technique analytique directe.

On procède en effet comme à [Ma1, §4], mais ici, l'analyse est plus facile puisqu'on est en dimension finie. Toutefois, on a une difficulté technique supplémentaire, puisque la suite spectrale ne dégénère en général pas en $\mathcal{E}_{2}$.

Cette Section est organisée de la façon suivante. Dans (a), on rappelle les notations et les hypothèses. Dans (b), comme à [Ma1, §4], on construit une 1-forme sur $\mathbf{R}_{+}^{*} \times \mathbf{R}_{+}^{*}$. Dans (c), on énonce une suite de résultats intermédiaires qui sont utilisés dans la preuve du Théorème 1.2. Dans (d), on montre le Théorème 1.2. Dans (e) et (f), on démontre les résultats intermédiaires.

On utilise les mêmes notations de [Ma1, §10(a)].

a) Le théorème principal. Soit $S$ une variété complexe de dimension $m$. Soit $\mathcal{E}=\left(\mathcal{E}^{p, q}\right)(0 \leq p, q \leq n)$ un bicomplexe de fibrés vectoriels holomorphes sur $S$. Soit $H(\mathcal{E})$ la cohomologie de $\mathcal{E}$. Soit $\left(\mathcal{E}_{r}, d_{r}\right)$ la suite spectrale induite par la filtration $F^{p} \mathcal{E}=\oplus_{p^{\prime} \geq p} \mathcal{E}^{p^{\prime}, \bullet}$. Soit $F^{\bullet} H(\mathcal{E})$ la filtration associée sur $H(\mathcal{E})$. On pose $\mathrm{Gr}^{p} H^{p+q}(\mathcal{E})=F^{p} H^{p+q}(\mathcal{E}) / F^{p+1} H^{p+q}(\mathcal{E})$. Then $\mathcal{E}_{\infty}^{p, q}=\mathrm{Gr}^{p} H^{p+q}(\mathcal{E})$. On suppose que pour $p, q, r \geq 0$, le rang des fibres $\mathcal{E}_{r}^{p, q}$ est localement constant. Alors les $\mathcal{E}_{r}^{p, q}$, $F^{p} H^{p+q}(\mathcal{E})$ sont des fibrés vectoriels holomorphes sur $S$.

Soit $h^{\mathcal{E}}=\oplus h^{\mathcal{E}^{p, q}}$ une métrique hermitienne sur $\mathcal{E}=\oplus \mathcal{E}^{p, q}$, telle que les $\mathcal{E}^{p, q}$ soient mutuellement orthogonaux. Soit $h^{H(\mathcal{E})}$ la métrique sur $H(\mathcal{E})$ induite par $h^{\mathcal{E}}$, en utilisant la théorie de Hodge.

Soient $d: \mathcal{E}^{p, q} \rightarrow \mathcal{E}^{p+1, q}, v: \mathcal{E}^{p, q} \rightarrow \mathcal{E}^{p, q+1}$ les différentielles du bicomplexe $\mathcal{E}$. Soit $v^{*}, d^{*}$ l'adjoint de $v, d$ pour $h^{\mathcal{E}}$. Alors on a

$$
[v, d]=\left[v^{*}, d^{*}\right]=0 .
$$

On pose

$$
D=d+d^{*}, \quad V=v+v^{*} .
$$

Soient $N_{H}, N_{V}$ les opérateurs de nombre sur $\mathcal{E}$, i.e. $N_{H}, N_{V}$ agissent sur $\mathcal{E}^{p, q}, \mathcal{E}_{r}^{p, q}$ par multiplication par $p, q$. Alors $N=N_{H}+N_{V}$ est l'opérateur de nombre total sur $\mathcal{E}$.

On va définir par récurrence une suite de sous-fibrés hermitiens de $\mathcal{E}, \mathcal{E}_{0}^{\prime} \supset \mathcal{E}_{1}^{\prime} \supset$ $\cdots \supset \mathcal{E}_{r}^{\prime} \supset \cdots$ tels que

$$
\mathcal{E}_{r}^{\prime} \simeq \mathcal{E}_{r}
$$

On pose

$$
\mathcal{E}_{0}^{\prime}=\mathcal{E}_{0}=\mathcal{E}
$$

Supposons qu'on a construit les $\mathcal{E}_{r^{\prime}}^{\prime}\left(r^{\prime} \leq r\right)$. Alors comme $\mathcal{E}_{r}^{\prime} \simeq \mathcal{E}_{r}$, l'opérateur $d_{r}$ agit $\operatorname{sur} \mathcal{E}_{r}^{\prime}$. Soit $d_{r}^{*}$ l'adjoint de $d_{r}$ pour la métrique de $\mathcal{E}_{r}^{\prime}$. On pose

$$
\begin{gathered}
D_{r}=d_{r}+d_{r}^{*}, \\
\mathcal{E}_{r+1}^{\prime}=\operatorname{Ker} D_{r} .
\end{gathered}
$$


Alors $\mathcal{E}_{r+1}^{\prime} \subset \mathcal{E}_{r}^{\prime}$, et $\mathcal{E}_{r+1}^{\prime}$ hérite d'une métrique de $\mathcal{E}_{r}^{\prime}$. Par la théorie de Hodge,

$$
\mathcal{E}_{r+1}^{\prime} \simeq \mathcal{E}_{r+1}
$$

Le résultat suivant est établi dans [BerB, Théorème 6.1].

Proposition 1.1. Pour $r \in \mathrm{N}$, $\mathcal{E}_{r}^{\prime}$ est naturellement scindé en une somme directe orthogonale $\mathcal{E}_{r}^{\prime}=\oplus_{p, q} \mathcal{E}_{r}^{\prime p, q}$, avec $\mathcal{E}_{r}^{\prime p, q} \subset \mathcal{E}^{p, q}$, de telle sorte que sous l'identification $\left(\mathcal{E}_{r}^{\prime}, d_{r}\right) \simeq\left(\mathcal{E}_{r}, d_{r}\right)$, on $a \mathcal{E}_{r}^{\prime p, q} \simeq \mathcal{E}_{r}^{p, q}$.

Désormais, on ne distingue plus $\mathcal{E}_{r}^{\prime}$ et $\mathcal{E}_{r}$. Donc on a

$$
\mathcal{E}=\mathcal{E}_{0} \supset \mathcal{E}_{1} \supset \mathcal{E}_{2} \supset \cdots
$$

Soit $h^{\mathcal{E}_{r}}(r \geq 0)$ la métrique sur $\mathcal{E}_{r}$ induite par la métrique $h^{\mathcal{E}}$.

Soit $P^{S}$ l'espace vectoriel de formes réelles sur $S$ qui sont la somme de formes $\mathcal{C}^{\infty}$ de type $(p, p)$. Soit $P^{S, 0}$ l'espace des $\alpha \in P^{S}$, qui s'écrivent sous la forme $\alpha=\partial \beta+\bar{\partial} \gamma$, où $\beta, \gamma$ sont des formes $\mathcal{C}^{\infty}$ sur $S$.

Soient $\widetilde{\operatorname{ch}}\left(\mathcal{E}, H(\mathcal{E}), h^{\mathcal{E}}, h^{H(\mathcal{E})}\right), \widetilde{\operatorname{ch}}\left(\mathcal{E}_{r}, \mathcal{E}_{r+1}, h^{\mathcal{E}_{r}}, h^{\mathcal{E}_{r+1}}\right), \tilde{\operatorname{ch}}\left(H(\mathcal{E}), \mathcal{E}_{\infty}, h^{H(\mathcal{E})}, h^{\mathcal{E}_{\infty}}\right) \in$ $P^{S} / P^{S, 0}$ des classes de Bott-Chern au sens de [BGS1], définies dans [Ma1, §10(a)].

THÉORÈme 1.2. On a l'égalité suivante

$$
\begin{array}{r}
\tilde{\operatorname{ch}}\left(\mathcal{E}, H(\mathcal{E}), h^{\mathcal{E}}, h^{H(\mathcal{E})}\right)=\Sigma_{r=0}^{+\infty} \tilde{\operatorname{ch}}\left(\mathcal{E}_{r}, \mathcal{E}_{r+1}, h^{\mathcal{E}_{r}}, h^{\mathcal{E}_{r+1}}\right) \\
-\tilde{\operatorname{ch}}\left(H(\mathcal{E}), \mathcal{E}_{\infty}, h^{H(\mathcal{E})}, h^{\mathcal{E}_{\infty}}\right) \quad \text { dans } \quad P^{S} / P^{S, 0} .
\end{array}
$$

PREUVE. Le reste de cette Section est consacré à la preuve du Théorème 1.2. $\mathrm{Q}$

Pour $r \geq 1$, soit $\mathcal{E}_{r}^{\perp}$ l'espace orthogonal à $\mathcal{E}_{r}$ dans $\mathcal{E}_{r-1}$. Pour $r \geq 1$, soit $p_{r}$ la projection orthogonale de $\mathcal{E}_{0}$ sur $\mathcal{E}_{r}$. On pose $p_{r}^{\perp}=1-p_{r}$. Alors $D_{r}$ agit sur $\mathcal{E}_{r+1}^{\perp}$ comme un opérateur inversible.

Soient $\nabla^{\mathcal{E}}, \nabla^{\mathcal{E}_{r}}$ les connexions holomorphes hermitiennes $\operatorname{sur}\left(\mathcal{E}, h^{\mathcal{E}}\right),\left(\mathcal{E}_{r}, h^{\mathcal{E}_{r}}\right)$. Soit $\bar{p}_{r}$ la projection orthogonale de $\mathcal{E}_{r}$ sur $\mathcal{E}_{r+1}$. Alors par [B2, Proposition 1.8], on a

$$
\nabla^{\mathcal{E}_{r+1}}=\bar{p}_{r} \nabla^{\mathcal{E}_{r}} \bar{p}_{r}
$$

Par récurrence, on a

$$
\nabla^{\mathcal{E}_{r}}=p_{r} \nabla^{\mathcal{E}} p_{r}
$$

b) Une 1-forme sur $\mathbf{R}_{+}^{*} \times \mathbf{R}_{+}^{*}$. Pour $u>0, T \geq 1, r \geq 0$, on pose

$$
\begin{array}{ll}
A_{T} & =D+T V, \\
\mathcal{D}_{3, u, T} & =\nabla^{\mathcal{E}}+u A_{T}, \\
\mathcal{D}_{r, u} & =\nabla^{\mathcal{E}_{r}}+u D_{r} .
\end{array}
$$

On définit aussi

$$
\mathcal{D}_{3, u, T}^{\prime}=\nabla^{\mathcal{E}^{\prime}}+u\left(d^{*}+T v^{*}\right), \quad \mathcal{D}_{3, u, T}^{\prime \prime}=\nabla^{\mathcal{E}^{\prime \prime}}+u(d+T v) .
$$

Soit $\varphi$ l'homomorphisme de $\Lambda\left(T_{\mathbf{R}}^{*} S\right)$ sur $\Lambda\left(T_{\mathbf{R}}^{*} S\right): \alpha \rightarrow(2 \pi i)^{\frac{-\operatorname{deg} \alpha}{2}} \alpha$.

DÉFINITION 1.3. Soit $\alpha_{u, T}$ une 1-forme à valeurs dans $P^{S}$ sur $\mathbf{R}_{+}^{*} \times \mathbf{R}_{+}^{*}$ :

$$
\alpha_{u, T}=d u \varphi \operatorname{Tr}_{s}\left[\frac{2 N}{u} \exp \left(-\mathcal{D}_{3, u, T}^{2}\right)\right]+d T \varphi \operatorname{Tr}_{s}\left[\frac{2 N_{V}}{T} \exp \left(-\mathcal{D}_{3, u, T}^{2}\right)\right]
$$


THÉORÈme 1.4. On a l'égalité suivante:

$$
\begin{array}{r}
d \alpha_{u, T}=\frac{2}{u} d u d T \varphi\left\{\bar{\partial} \frac{\partial}{\partial b} \operatorname{Tr}_{s}\left[\left[\mathcal{D}_{3, u, T}^{\prime}, N\right] \exp \left(-\mathcal{D}_{3, u, T}^{2}-\frac{b}{T} N_{V}\right)\right]_{b=0}\right. \\
-\partial \frac{\partial}{\partial b} \operatorname{Tr}_{s}\left[\left[\mathcal{D}_{3, u, T}^{\prime \prime}, N\right] \exp \left(-\mathcal{D}_{3, u, T}^{2}-\frac{b}{T} N_{V}\right)\right]_{b=0} \\
\left.-\bar{\partial} \partial \frac{\partial}{\partial b} \operatorname{Tr}_{s}\left[N \exp \left(-\mathcal{D}_{3, u, T}^{2}-\frac{b}{T} N_{V}\right)\right]_{b=0}\right\} .
\end{array}
$$

Preuve. La preuve est la même que dans [BKö, Théorème 2.9].

c) Des résultats intermédiaires. Pour $T>0$, on pose

$$
h_{T}^{\mathcal{E}}=\oplus_{p, q} T^{2 q} h^{\mathcal{E}^{p, q}}
$$

Alors $h_{T}^{\mathcal{E}}$ est une métrique hermitienne sur $\mathcal{E}=\oplus_{p, q} \mathcal{E}^{p, q}$. Alors l'adjoint de $v$ pour la métrique $h_{T}^{\mathcal{E}}$ est donné par $T^{2} v^{*}$. On pose

$$
D_{T}^{\prime}=D+v+T^{2} v^{*}
$$

Alors on a un isomorphisme canonique $\mathcal{C}^{\infty}$ de fibrés vectoriels sur $S$

$$
H(\mathcal{E}) \simeq \operatorname{Ker} D_{T}^{\prime}
$$

Soit $h_{T}^{H(\mathcal{E})}$ la métrique hermitienne sur $H(\mathcal{E})$ induite par l'identification (1.16). Soit $\nabla_{T}^{H(\mathcal{E})}$ la connexion holomorphe hermitienne sur $\left(H(\mathcal{E}), h_{T}^{H(\mathcal{E})}\right)$. Soit $P_{T}$ la projection orthogonale de $\mathcal{E}$ sur $\operatorname{Ker} D_{T}^{\prime}$ associée à $h_{T}^{\mathcal{E}}$.

ThÉORÈme 1.5. i) Pour tout $u>0$,

$$
\lim _{T \rightarrow+\infty} \varphi \operatorname{Tr}_{s}\left[N \exp \left(-\mathcal{D}_{3, u, T}^{2}\right)\right]=\varphi \operatorname{Tr}_{s}\left[N \exp \left(-\mathcal{D}_{1, u}^{2}\right)\right]
$$

ii) Pour tout $u>0$, il existe $C>0$ tel que pour tout $T \geq 1$,

$$
\left|\varphi \operatorname{Tr}_{s}\left[N_{V} \exp \left(-\mathcal{D}_{3, u, T}^{2}\right)\right]-\varphi \operatorname{Tr}_{s}\left[N_{V} \exp \left(-\mathcal{D}_{1, u}^{2}\right)\right]\right| \leq \frac{C}{T}
$$

iii) Pour tout $0<u_{1}<u_{2}$ fixés, il existe $C>0$ tel que pour $u \in\left[u_{1}, u_{2}\right], T \geq 1$,

$$
\left|\varphi \operatorname{Tr}_{s}\left[N \exp \left(-\mathcal{D}_{3, u, T}^{2}\right)\right]\right| \leq C
$$

Pour $r>0$, on pose

$$
\begin{aligned}
& \operatorname{ch}^{\prime}\left(\mathcal{E}_{r}, h^{\mathcal{E}_{r}}\right)=\varphi \operatorname{Tr}_{s}\left[N \exp \left(-\nabla^{\mathcal{E}_{r}, 2}\right)\right] \\
& \operatorname{ch}^{\prime}\left(H(\mathcal{E}), h^{H(\mathcal{E})}\right)=\Sigma_{i}(-1)^{i} i \operatorname{ch}\left(H^{i}(\mathcal{E}), h^{H^{i}(\mathcal{E})}\right) .
\end{aligned}
$$

On rappelle qu'on a défini $\zeta\left(\mathcal{E}_{r}, h^{\mathcal{E}_{r}}\right) \in P^{S}$ dans [Ma1, Définition 10.3]. Par [Ma1, Proposition 10.4], on a

$$
\zeta\left(\mathcal{E}_{r}, h^{\mathcal{E}_{r}}\right)=\tilde{\operatorname{ch}}\left(\mathcal{E}_{r}, \mathcal{E}_{r+1}, h^{\mathcal{E}_{r}}, h^{\mathcal{E}_{r+1}}\right) \quad \text { dans } \quad P^{S} / P^{S, 0} .
$$


THÉORÈME 1.6. On a l'identité suivante

$$
\begin{aligned}
\lim _{T \rightarrow+\infty}\left\{\int_{1}^{+\infty} 2\left\{\varphi \operatorname{Tr}_{s}\left[N \exp \left(-\mathcal{D}_{3, u, T}^{2}\right)\right]-\varphi \operatorname{Tr}_{s}\left[N \exp \left(-\nabla_{T}^{H(\mathcal{E}), 2}\right)\right]\right\} \frac{d u}{u}\right. \\
\left.-2 \Sigma_{r \geq 2}(r-1)\left[\operatorname{ch}^{\prime}\left(\mathcal{E}_{r}, h^{\mathcal{E}_{r}}\right)-\operatorname{ch}^{\prime}\left(\mathcal{E}_{r+1}, h^{\mathcal{E}_{r+1}}\right)\right] \log T\right\} \\
=2 \int_{1}^{+\infty}\left\{\varphi \operatorname{Tr}_{s}\left[N \exp \left(-\mathcal{D}_{1, u}^{2}\right)\right]-\operatorname{ch}^{\prime}\left(\mathcal{E}_{2}, h^{\mathcal{E}_{2}}\right)\right\} \frac{d u}{u} \\
-\Sigma_{r \geq 2} \zeta\left(\mathcal{E}_{r}, h^{\mathcal{E}_{r}}\right)+\Gamma^{\prime}(1)\left[\operatorname{ch}^{\prime}\left(\mathcal{E}_{2}, h^{\mathcal{E}_{2}}\right)-\operatorname{ch}^{\prime}\left(\mathcal{E}_{\infty}, h^{\mathcal{E}_{\infty}}\right)\right]
\end{aligned}
$$

THÉORÈME 1.7. Quand $T \rightarrow+\infty$, on a

$$
\varphi \operatorname{Tr}_{s}\left[N_{V} \exp \left(-\nabla_{T}^{H(\mathcal{E}), 2}\right)\right]=\varphi \operatorname{Tr}_{s}\left[N_{V} \exp \left(-\nabla^{\mathcal{E}_{\infty}, 2}\right)\right]+O\left(\frac{1}{T}\right)
$$

De plus

$$
\begin{array}{r}
\int_{1}^{+\infty}\left\{\varphi \operatorname{Tr}_{s}\left[N_{V} \exp \left(-\nabla_{T}^{H(\mathcal{E}), 2}\right)\right]-\varphi \operatorname{Tr}_{s}\left[N_{V} \exp \left(-\nabla^{\mathcal{E}_{\infty}, 2}\right)\right]\right\} \frac{d T}{T} \\
=-\frac{1}{2} \tilde{\operatorname{ch}}\left(H(\mathcal{E}), \mathcal{E}_{\infty}, h^{H(\mathcal{E})}, h^{\mathcal{E}_{\infty}}\right) \text { dans } \quad P^{S} / P^{S, 0}
\end{array}
$$

PrEUVE. Les preuves des Théorèmes 1.5 et 1.6 sont différées à la Section 1(e), et la preuve du Théorème 1.7 est différée à la Section $1(\mathrm{f})$.

d) Preuve du Théorème 1.2. Pour montrer le Théorème 1.2, on utilise la même méthode qu'à [Ma1, §4]. On note aussi $I_{j}^{i}$ les termes correspondants définis dans [Ma1, §4(c)]. En utilisant [Ma1, (10.12)], (1.21), les Théorèmes 1.5-1.7 et en procédant comme dans [Ma1, §4], on a

$$
\begin{aligned}
I_{1}^{3}= & -\Sigma_{r=1}^{+\infty} \tilde{\operatorname{ch}}\left(\mathcal{E}_{r}, \mathcal{E}_{r+1}, h^{\mathcal{E}_{r}}, h^{\mathcal{E}_{r+1}}\right)+\Gamma^{\prime}(1)\left[\operatorname{ch}^{\prime}\left(\mathcal{E}_{1}, h^{\mathcal{E}_{1}}\right)-\operatorname{ch}^{\prime}\left(\mathcal{E}_{\infty}, h^{\mathcal{E}_{\infty}}\right)\right] \\
I_{2}^{3}= & \tilde{\operatorname{ch}}\left(H(\mathcal{E}), \mathcal{E}_{\infty}, h^{H(\mathcal{E})}, h^{\mathcal{E}_{\infty}}\right) \\
I_{3}^{3}= & \tilde{\operatorname{ch}}\left(\mathcal{E}, H(\mathcal{E}), h^{\mathcal{E}}, h^{H(\mathcal{E})}\right)+\Gamma^{\prime}(1)\left[-\operatorname{ch}^{\prime}\left(\mathcal{E}, h^{\mathcal{E}}\right)\right. \\
& \left.\quad+\operatorname{ch}^{\prime}\left(H(\mathcal{E}), h^{H(\mathcal{E})}\right)\right] \\
I_{4}^{3}= & -\tilde{\operatorname{ch}}\left(\mathcal{E}, \mathcal{E}_{1}, h^{\mathcal{E}}, h^{\mathcal{E}_{1}}\right)+\Gamma^{\prime}(1)\left\{\varphi \operatorname{Tr}_{s}\left[N_{V} \exp \left(-\nabla^{\mathcal{E}, 2}\right)\right]\right. \\
& \left.-\varphi \operatorname{Tr}_{s}\left[N_{V} \exp \left(-\nabla^{\mathcal{E}_{1}, 2}\right)\right]\right\}
\end{aligned}
$$

En combinant la preuve du Théorème 1.6, et [B4, §6.6-6.8], on peut traiter aussi le terme à droite de (1.14). On a ainsi

$$
\Sigma_{i=1}^{4} I_{i}^{3}=0 \quad \text { dans } \quad P^{S} / P^{S, 0} .
$$

D'après [Ma1, $\S 10(a)]$, on a aussi

$$
\begin{gathered}
\operatorname{ch}^{\prime}\left(H(\mathcal{E}), h^{H(\mathcal{E})}\right)-\operatorname{ch}^{\prime}\left(\mathcal{E}_{\infty}, h^{\mathcal{E}_{\infty}}\right) \in P^{S, 0} \\
\varphi \operatorname{Tr}_{s}\left[N_{V} \exp \left(-\nabla^{\mathcal{E}, 2}\right)\right]-\varphi \operatorname{Tr}_{s}\left[N_{V} \exp \left(-\nabla^{\mathcal{E}_{1}, 2}\right)\right] \in P^{S, 0}
\end{gathered}
$$

Par (1.24)-(1.26), on obtient le Théorème 1.2. 
e) Preuves des Théorèmes 1.5 et 1.6. Soit $B_{T}(T \in[1,+\infty])$ une famille de tenseurs, on notera que pour $l>0$, quand $T \rightarrow+\infty$

$$
B_{T}=O\left(\frac{1}{T^{l}}\right)
$$

si pour tout $k \in \mathbf{N}, K \subset S$ compact, il existe $c>0$ tel que pour $T \geq 1$, le sup des normes de $B_{T}$ et de ses dérivées d'ordre $\leq k$ sur $K$ est dominé par $\frac{c}{T^{r}}$.

Dans la suite, les convergences des formes sur $S$ sont au sens de la convergence uniforme sur tout compact $K \subset S$. Pour simplifier les énoncés, on suppose désormais que $S$ est compacte.

Pour $r \geq 0, x \in S$, soit $\operatorname{Sp} D_{r, x}$ le spectre de $D_{r, x}$, soit $\operatorname{Sp} D_{r, x}^{\neq 0}$ l'ensemble des valeurs propres non nulles de $D_{r, x}$. Désormais, on fixe $c_{1}>c_{2}>0$ tels que pour $r \in \mathbf{N}$

$$
\cup_{\substack{r \geq 0 \\ x \in S}} \operatorname{Sp} D_{r, x}^{\neq 0} \subset\left\{s \in \mathbf{R}, \sqrt{c_{1}}<|s|<\sqrt{c_{2}}\right\}
$$

Soit

$$
U_{1}=\left\{\lambda \in \mathbf{C}, \frac{\sqrt{c_{1}}}{2} \leq|\lambda| \leq \sqrt{c_{1}} \text {, ou } \quad \sqrt{c_{2}} \leq|\lambda| \leq 2 \sqrt{c_{2}}\right\} .
$$

Proposition 1.8. Il existe $T_{0}>0$ tel que pour tout $T \geq T_{0}, r \geq 1, \lambda \in U_{1}$, $\left(\lambda-T^{r-1} A_{T}\right)^{-1}$ existe, et quand $T \rightarrow+\infty$

$$
\left(\lambda-T^{r-1} A_{T}\right)^{-1}=p_{r}\left(\lambda-D_{r}\right)^{-1} p_{r}+O\left(\frac{1}{T}\right) .
$$

Preuve. Comme $p_{r^{\prime}}\left(r^{\prime} \geq 1\right)$ est $\mathcal{C}^{\infty}$ sur $S$, en procédant comme en [BerB, Théorème 6.2], on sait que les applications définies comme dans [BerB, Théorème $6.2]$ sont $\mathcal{C}^{\infty}$ sur $S$. En utilisant [BerB, (6.49)], on obtient la Proposition.

Par (1.29), pour $r \geq 1$, les valeurs propres non nulles de $A_{T}$ qui se comportent comme $O\left(\frac{1}{T^{r-1}}\right)$ sont en correspondance biunivoque avec les valeurs propres non nulles de $D_{r}$. Pour $T \gg 1$, on pose

$$
p_{r, T}=\frac{1}{2 \pi i} \int_{\left\{\lambda \in \mathbf{C},|\lambda|=\sqrt{c_{2}}\right\}}\left(\lambda-T^{r-1} A_{T}\right)^{-1} d \lambda, \quad p_{r, T}^{\perp}=1-p_{r, T} .
$$

Alors en utilisant la Proposition 1.8, quand $T \rightarrow+\infty$, on a

$$
p_{r, T}=p_{r}+O\left(\frac{1}{T}\right)
$$

Pour $T \geq 1, u>0$, on pose

$$
B_{r, u, T}=\nabla^{\mathcal{E}}+u T^{r-1} A_{T} .
$$

THÉORÈme 1.9. Il existe $T_{0}>0$ tel que pour tout $\lambda \in U_{1}, T \geq T_{0}, r \geq 1$, $\left(\lambda^{2}-B_{r, 1, T}^{2}\right)^{-1}$ existe, et

$$
\left(\lambda^{2}-B_{r, 1, T}^{2}\right)^{-1}=p_{r}\left(\lambda^{2}-\mathcal{D}_{r, 1}^{2}\right)^{-1} p_{r}+O\left(\frac{1}{T}\right) .
$$

Preuve. Comme en [Ma1, Proposition 5.12], on a

$$
\operatorname{Sp}\left(B_{r, u, T}^{2}\right)=\operatorname{Sp}\left(T^{2(r-1)} A_{T}^{2}\right) .
$$


D'après la Proposition 1.8, (1.34), il existe $T_{0}>0$ tel que pour tout $T \geq T_{0}, r \geq 1, \lambda \in$ $U_{1},\left(\lambda^{2}-B_{r, 1, T}^{2}\right)^{-1}$ existe. On pose

$$
\begin{aligned}
& E_{T}=p_{r, T} B_{r, 1, T}^{2} p_{r, T}, \quad F_{T}=p_{r, T} B_{r, 1, T}^{2} p_{r, T}^{\perp} \\
& G_{T}=p_{r, T}^{\perp} B_{r, 1, T}^{2} p_{r, T}, \quad H_{T}=p_{r, T}^{\perp} B_{r, 1, T}^{2} p_{r, T}^{\perp}
\end{aligned}
$$

Alors en utilisant (1.10), (1.29) et (1.30), quand $T \rightarrow+\infty$, on a

$$
\begin{aligned}
& T^{r-1} A_{T} p_{r, T}=p_{r, T} T^{r-1} A_{T}=p_{r} D_{r} p_{r}+O\left(\frac{1}{T}\right) \\
& E_{T}=p_{r} D_{r}^{2} p_{r}+p_{r}\left[D_{r}, \nabla^{\mathcal{E}_{r}}\right] p_{r}+p_{r} \nabla^{\mathcal{E}, 2} p_{r}+O\left(\frac{1}{T}\right), \\
& F_{T}=p_{r, T}\left[\nabla^{\mathcal{E}}, T^{r-1} A_{T}\right] p_{r, T}^{\perp}+O(1), \\
& G_{T}=p_{r, T}^{\perp}\left[\nabla^{\mathcal{E}}, T^{r-1} A_{T}\right] p_{r, T}+O(1) .
\end{aligned}
$$

$\operatorname{Par}(1.29)$ et $(1.30)$, quand $T \rightarrow+\infty$, on a aussi

$$
\begin{aligned}
& p_{r, T}^{\perp}\left(\lambda-T^{r-1} A_{T}\right)^{-1}=\left(\lambda-T^{r-1} A_{T}\right)^{-1} p_{r, T}^{\perp}=O\left(\frac{1}{T}\right), \\
& T^{r-1} A_{T} p_{r, T}^{\perp}\left(\lambda-T^{r-1} A_{T}\right)^{-1} \\
& \quad=\left(\lambda-T^{r-1} A_{T}\right)^{-1} p_{r, T}^{\perp} T^{r-1} A_{T}=-p_{r}^{\perp}+O\left(\frac{1}{T}\right) .
\end{aligned}
$$

D'après (1.37), quand $T \rightarrow+\infty$, on a

$$
\begin{aligned}
(\lambda- & \left.T^{r-1} A_{T}\right)^{-1} p_{r, T}^{\perp}\left[\nabla^{\mathcal{E}}, T^{r-1} A_{T}\right] p_{r, T}^{\perp}\left(\lambda+T^{r-1} A_{T}\right)^{-1} \\
= & {\left[\left(\lambda-T^{r-1} A_{T}\right)^{-1} p_{r, T}^{\perp} T^{r-1} A_{T}\right] \nabla^{\mathcal{E}} p_{r, T}^{\perp}\left(\lambda+T^{r-1} A_{T}\right)^{-1} } \\
& +\left(\lambda-T^{r-1} A_{T}\right)^{-1} p_{r, T}^{\perp} \nabla^{\mathcal{E}}\left[T^{r-1} A_{T} p_{r, T}^{\perp}\left(\lambda+T^{r-1} A_{T}\right)^{-1}\right] \\
= & O\left(\frac{1}{T}\right) .
\end{aligned}
$$

Par (1.32) et (1.38), pour $T \geq T_{0}, \lambda \in U_{1}$, on a

$$
\begin{aligned}
\left(\lambda^{2}-H_{T}\right)^{-1}= & \sum_{i=0}^{2 m} p_{r, T}^{\perp}\left(\lambda+T^{r-1} A_{T}\right)^{-1}\left\{\left(\lambda-T^{r-1} A_{T}\right)^{-1}\right. \\
& p_{r, T}^{\perp}\left(\nabla^{\mathcal{E}, 2}+\left[\nabla^{\mathcal{E}}, T^{r-1} A_{T}\right]\right) p_{r, T}^{\perp} \\
& \left.\left(\lambda+T^{r-1} A_{T}\right)^{-1}\right\}^{i}\left(\lambda-T^{r-1} A_{T}\right)^{-1} p_{r, T}^{\perp} \\
= & p_{r, T}^{\perp}\left(\lambda^{2}-T^{2(r-1)} A_{T}^{2}\right)^{-1} p_{r, T}^{\perp} \\
& +p_{r, T}^{\perp}\left(\lambda+T^{r-1} A_{T}\right)^{-1} O\left(\frac{1}{T}\right)\left(\lambda-T^{r-1} A_{T}\right)^{-1} p_{r, T}^{\perp}
\end{aligned}
$$

En utilisant (1.36), (1.37) et (1.39), pour $T \geq T_{0}$, on a

$$
\begin{gathered}
F_{T}\left(\lambda^{2}-H_{T}\right)^{-1}=p_{r, T}\left[\nabla^{\mathcal{E}}, T^{r-1} A_{T}\right] p_{r, T}^{\perp}\left(\lambda^{2}-T^{2(r-1)} A_{T}^{2}\right)^{-1} p_{r, T}^{\perp}+O\left(\frac{1}{T}\right) \\
=\left(p_{r, T} T^{r-1} A_{T}\right) \nabla^{\mathcal{E}}\left(p_{r, T}^{\perp}\left(\lambda^{2}-T^{2(r-1)} A_{T}^{2}\right)^{-1} p_{r, T}^{\perp}\right) \\
\quad+p_{r, T} \nabla^{\mathcal{E}}\left(T^{r-1} A_{T} p_{r, T}^{\perp}\left(\lambda^{2}-T^{2(r-1)} A_{T}^{2}\right)^{-1} p_{r, T}^{\perp}\right)+O\left(\frac{1}{T}\right)=O\left(\frac{1}{T}\right) .
\end{gathered}
$$

De même, on a

$$
\left(\lambda^{2}-H_{T}\right)^{-1} G_{T}=O\left(\frac{1}{T}\right) .
$$

Par (1.36), (1.37) et (1.39), on a

$$
F_{T}\left(\lambda^{2}-H_{T}\right)^{-1} G_{T}=-p_{r}\left(\nabla^{\mathcal{E}} p_{r}^{\perp}\right)\left(\nabla^{\mathcal{E}} p_{r}\right)+O\left(\frac{1}{T}\right) .
$$


D'après (1.10), (1.36) et (1.42), on a

$$
E_{T}+F_{T}\left(\lambda^{2}-H_{T}\right)^{-1} G_{T}=p_{r} \mathcal{D}_{r, 1}^{2} p_{r}+O\left(\frac{1}{T}\right)
$$

On pose

$$
M_{T, \lambda}=\lambda^{2}-E_{T}-F_{T}\left(\lambda^{2}-H_{T}\right)^{-1} G_{T} .
$$

$\operatorname{Par}[\operatorname{BerB},(5.85)]$, on peut écrire $\left(\lambda^{2}-B_{r, 1, T}^{2}\right)^{-1}$ sous forme matricielle:

$$
\begin{aligned}
& \left(\lambda^{2}-B_{r, 1, T}^{2}\right)^{-1} \\
& \quad=\left[\begin{array}{lc}
M_{T, \lambda}^{-1} & M_{T, \lambda}^{-1} F_{T}\left(\lambda^{2}-H_{T}\right)^{-1} \\
\left(\lambda^{2}-H_{T}\right)^{-1} G_{T} M_{T, \lambda}^{-1} & \left(\lambda^{2}-H_{T}\right)^{-1}\left(1+G_{T} M_{T, \lambda}^{-1} F_{T}\left(\lambda^{2}-H_{T}\right)^{-1}\right)
\end{array}\right]
\end{aligned}
$$

Par (1.31), (1.40), (1.41), (1.43) et (1.45), on a (1.33).

Soit $\Delta$ le contour orienté ci-dessous, et soit $\delta$ le cercle dans $\mathbf{C}$ de centre 0 et de rayon $c_{1} / 4$.

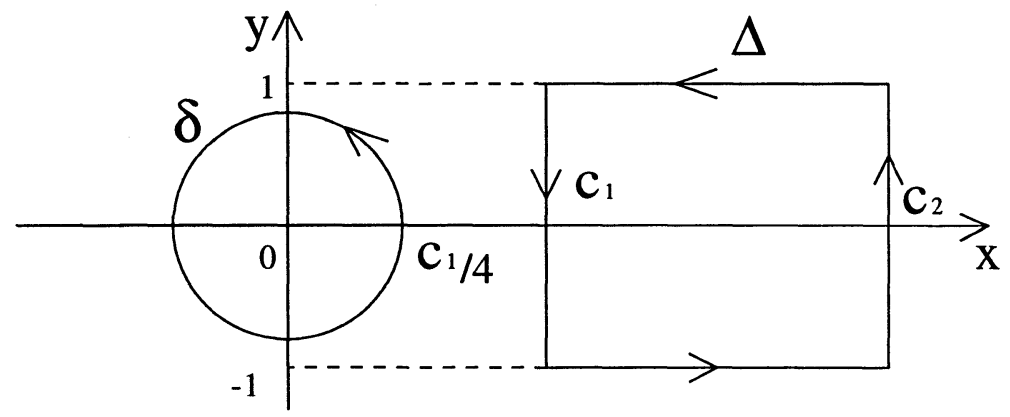

Les contours $\Delta$ et $\delta$ sont les bords des domaines $\Delta^{\prime}$ et $\delta^{\prime}$. Alors d'après notre choix de $c_{1}$ et $c_{2}$ et (1.29), quand $T$ est assez grand, on a

$$
\operatorname{Sp}\left(\mathcal{D}_{3,1, T}^{2}\right)=\operatorname{Sp}\left(A_{T}^{2}\right) \subset \frac{\delta^{\prime}}{T^{2(n-1)}} \cup \cup_{i=0}^{n} \frac{\Delta^{\prime}}{T^{2(i-1)}} .
$$

Dans la suite, on va exprimer les supertraces des termes contenant $\mathcal{D}_{3,1, T}^{2}$ comme une somme de supertraces indexées par les éléments du spectre de $\mathcal{D}_{3,1, T}^{2}$ qui sont inclus dans l'un des domaines du membre de droite de (1.46).

Pour $u>0$, soit $\psi_{u}: \Lambda\left(T_{\mathbf{R}}^{*} S\right) \rightarrow \Lambda\left(T_{\mathbf{R}}^{*} S\right)$ l'application $\alpha \rightarrow u^{-\operatorname{deg} \alpha} \alpha$. Pour $1 \leq r \leq n, T \geq T_{0}$, on pose

$$
\begin{aligned}
& F_{r, u, T}=\frac{1}{2 \pi i} \psi_{u} \varphi \operatorname{Tr}_{s}\left[N \int_{\Delta} e^{-u^{2} \lambda}\left(\lambda-B_{r, 1, T}^{2}\right)^{-1} d \lambda\right] \\
& F_{r, u, \infty}=\frac{1}{2 \pi i} \psi_{u} \varphi \operatorname{Tr}_{s}\left[N \int_{\Delta} e^{-u^{2} \lambda}\left(\lambda-\mathcal{D}_{r, 1}^{2}\right)^{-1} d \lambda\right] \\
& G_{r, u, T}=\frac{1}{2 \pi i} \psi_{u} \varphi \operatorname{Tr}_{s}\left[N \int_{\delta} e^{-u^{2} \lambda}\left(\lambda-B_{r, 1, T}^{2}\right)^{-1} d \lambda\right] \\
& G_{r, u, \infty}=\frac{1}{2 \pi i} \psi_{u} \varphi \operatorname{Tr}_{s}\left[N \int_{\delta} e^{-u^{2} \lambda}\left(\lambda-\mathcal{D}_{r, 1}^{2}\right)^{-1} d \lambda\right]
\end{aligned}
$$


Alors, on a

$$
G_{r, u, \infty}+F_{r, u, \infty}=\varphi \operatorname{Tr}_{s}\left[N \exp \left(-\mathcal{D}_{r, u}^{2}\right)\right] .
$$

Proposition 1.10. i) Il existe $C_{1}>0, C>0, T_{0}>0$ tels que pour $u \geq 1, T \geq$ $T_{0}, 1 \leq r \leq n$,

$$
\left|F_{r, u, T}-F_{r, u, \infty}\right| \leq \frac{C}{T} e^{-C_{1} u}
$$

ii) Il existe des formes $a_{r, i, T}, b_{r, i, T}\left(T \in\left[T_{0},+\infty\right], 1 \leq r \leq n,-2 m \leq i \leq 0\right), \mathcal{C}^{\infty}$ sur $S$, telles que quand $u \rightarrow 0$, uniformément en $T \geq T_{0}, 1 \leq r \leq n$, on a

$$
\begin{aligned}
& F_{r, u, T}=\Sigma_{i=-2 m}^{0} a_{r, i, T} u^{i}+O(u), \\
& G_{r, u, T}=\Sigma_{i=-2 m}^{0} b_{r, i, T} u^{i}+O(u) .
\end{aligned}
$$

Et

$$
\begin{aligned}
& G_{r, u, \infty}=-\Sigma_{i=-2 m}^{-1} a_{r, i, \infty} u^{i}+\operatorname{ch}^{\prime}\left(\mathcal{E}_{r+1}, h^{\mathcal{E}_{r+1}}\right) \\
& G_{n, u, T}=\Sigma_{i=-2 m}^{0} b_{n, i, T} u^{i} .
\end{aligned}
$$

iii) Pour $1 \leq r \leq n$, on $a$

$$
\begin{aligned}
& a_{r, 0, \infty}=\operatorname{ch}^{\prime}\left(\mathcal{E}_{r}, h^{\mathcal{E}_{r}}\right)-\operatorname{ch}^{\prime}\left(\mathcal{E}_{r+1}, h^{\mathcal{E}_{r+1}}\right) \\
& b_{n, 0, T}=\varphi \operatorname{Tr}_{s}\left[N \exp \left(-\nabla_{T}^{H(\mathcal{E}), 2}\right)\right]
\end{aligned}
$$

De plus, pour $1 \leq r \leq n$, quand $T \rightarrow+\infty$, on a

$$
\begin{aligned}
& a_{r, i, T}=a_{r, i, \infty}+O\left(\frac{1}{T}\right) \text { pour } i \leq 0, \\
& b_{r, i, T}=-a_{r, i, \infty}+O\left(\frac{1}{T}\right) \text { pour } i<0, \\
& b_{n, i, T} T^{-(n-1) i}+\Sigma_{r=2}^{n} a_{r, i, T} T^{-(r-1) i}=b_{1, i, T} \text { pour } i<0 .
\end{aligned}
$$

Preuve. i) Pour $1 \leq r \leq n, T \in\left[T_{0},+\infty\right]$, on pose

$$
f_{r, u, T}=\psi_{u}^{-1} F_{r, u, T}, \quad g_{r, u, T}=\psi_{u}^{-1} G_{r, u, T} .
$$

Alors $f_{r, u, T}$ et $g_{r, u, T}$ sont des fonctions holomorphes en $u \in \mathbf{C}$.

D'après le Théorème 1.9 , il existe $c, C>0$ tels que pour $u>1, T \geq T_{0}$, on a

$$
\begin{gathered}
\left|f_{r, u, T}-f_{r, u, \infty}\right| \leq C \int_{\Delta} e^{-c_{1} u^{2}}\left|\left(\lambda-B_{r, 1, T}^{2}\right)^{-1}-\left(\lambda-\mathcal{D}_{r, 1}^{2}\right)^{-1}\right| d \lambda \\
\leq \frac{C}{T} e^{-c u^{2}}
\end{gathered}
$$

Par (1.54), (1.55), on a (1.49).

ii) $\bullet$ On a

$$
e^{-u^{2} \lambda}=\Sigma_{k=0}^{\infty}(-1)^{k} \frac{\left(u^{2} \lambda\right)^{k}}{k !}
$$

En utilisant le Théorème $1.9,(1.47)$ et $(1.56)$, on a (1.50) et la première équation de (1.53). On a aussi

$$
b_{r, i, T}=b_{r, i, \infty}+O\left(\frac{1}{T}\right)
$$


- Comme à l'intérieur de $\delta^{\prime}, 0$ est l'unique valeur propre possible de l'opérateur $D_{r}^{2}$, pour $u \in \mathbf{C},|u| \geq 1$, on a

$$
G_{r, u, \infty}=\frac{1}{2 \pi i} \varphi \operatorname{Tr}_{s}\left[N \int_{\delta} e^{-\lambda}\left(\lambda-\mathcal{D}_{r, u}^{2}\right)^{-1} d \lambda\right]
$$

Par le même argument que dans [B4, Théorème 9.29], il existe des formes $b_{r, i, \infty}$ $(-2 m \leq i \leq 0), \mathcal{C}^{\infty}$ sur $S$, telles que pour $u \in \mathbf{C}$, on a

$$
G_{r, u, \infty}=\Sigma_{i=-2 m}^{0} b_{r, i, \infty} u^{i} .
$$

En utilisant [BeGeV, Théorème 9.2], (1.58) et (1.59), on a

$$
\begin{aligned}
& b_{r, 0, \infty}=\lim _{u \rightarrow+\infty} G_{r, u, \infty} \\
& =\frac{1}{2 \pi i} \varphi \operatorname{Tr}_{s}\left[p_{r+1} N p_{r+1} \int_{\delta} e^{-\lambda}\left(\lambda-\nabla^{\mathcal{E}_{r+1,2}}\right)^{-1} d \lambda\right]=\operatorname{ch}^{\prime}\left(\mathcal{E}_{r+1}, h^{\mathcal{E}_{r+1}}\right) .
\end{aligned}
$$

Par (1.48), (1.50) et (1.59), on a

$$
\Sigma_{i=-2 m}^{0}\left(b_{r, i, \infty}+a_{r, i, \infty}\right) u^{i}+O(u)=\varphi \operatorname{Tr}_{s}\left[N \exp \left(-\mathcal{D}_{r, u}^{2}\right)\right] .
$$

En comparant les coefficients de $u^{i}$ de (1.61), on a

$$
\begin{aligned}
& b_{r, 0, \infty}+a_{r, 0, \infty}=\operatorname{ch}^{\prime}\left(\mathcal{E}_{r}, h^{\mathcal{E}_{r}}\right), \\
& b_{r, i, \infty}=-a_{r, i, \infty} \text { pour } i<0 .
\end{aligned}
$$

Par (1.59), (1.60) et (1.62), on a la première équation de (1.51) et celle de (1.52).

D'après (1.57) et (1.62), on a aussi la deuxième équation de (1.53).

- Comme à l'intérieur de $\delta^{\prime}, 0$ est l'unique valeur propre possible de l'opérateur $T^{2(n-1)} A_{T}^{2}$, pour $u \in \mathbf{C},|u| \geq 1$, on a

$$
G_{n, u, T}=\frac{1}{2 \pi i} \varphi \operatorname{Tr}_{s}\left[N \int_{\delta} e^{-\lambda}\left(\lambda-B_{n, u, T}^{2}\right)^{-1} d \lambda\right] .
$$

Par le même argument que dans [B4, Théorème 9.29], on a la deuxième équation de (1.51).

Pour $T$ fixé, en utilisant $[\mathrm{BeGeV}$, Théorème 9.2] et (1.63), on a la deuxième équation de (1.52).

iii) D'après (1.46), pour $T \gg 1$, on a

$$
\begin{aligned}
G_{1, u, T}= & \sum_{r=2}^{n} \frac{1}{2 \pi i} \psi_{u} \varphi \operatorname{Tr}_{s}\left[N \int_{\frac{\Delta}{T^{2(r-1)}}} e^{-u^{2} \lambda}\left(\lambda-\mathcal{D}_{3,1, T}^{2}\right)^{-1} d \lambda\right] \\
& +\frac{1}{2 \pi i} \psi_{u} \varphi \operatorname{Tr}_{s}\left[N \int_{\frac{\delta}{T^{2(n-1)}}} e^{-u^{2} \lambda}\left(\lambda-\mathcal{D}_{3,1, T}^{2}\right)^{-1} d \lambda\right] \\
= & \sum_{r=2}^{n} F_{r, T^{-r+1} u, T}+G_{n, T^{-n+1} u, T} .
\end{aligned}
$$

En comparant (1.50), (1.51) et (1.64), on a la troisième équation de (1.53).

On a bien démontré la Proposition 1.10.

PREUVE du Théorème 1.5: Par (1.11), on a

$$
\varphi \operatorname{Tr}_{s}\left[N \exp \left(-\mathcal{D}_{3, u, T}^{2}\right)\right]=\psi_{u} \varphi \operatorname{Tr}_{s}\left[N \exp \left(-u^{2} \mathcal{D}_{3,1, T}^{2}\right)\right]
$$


Par (1.46), il existe $T_{0}>1$, tel que pour $T \geq T_{0}$, on a

$$
\exp \left(-u^{2} \mathcal{D}_{3,1, T}^{2}\right)=\frac{1}{2 \pi i} \int_{\delta \cup \Delta \cup T^{2} \Delta} \frac{\exp \left(-u^{2} \lambda\right)}{\lambda-\mathcal{D}_{3,1, T}^{2}} d \lambda
$$

Par (1.46), il existe aussi $C>0$ tel que pour $T \geq T_{0}$, on a

$$
\left|\int_{T^{2} \Delta} \frac{\exp \left(-u^{2} \lambda\right)}{\lambda-\mathcal{D}_{3,1, T}^{2}} d \lambda\right| \leq C e^{-T^{2} u^{2} c_{1}} .
$$

Par (1.33), (1.65) et (1.66), on a le Théorème 1.5.

PreUVE du Théorème 1.6: $\operatorname{Par}(1.33),(1.47),(1.66)$, il existe $C>0$ tel que pour $u \geq 1, T \geq T_{0}$, on a

$$
\begin{aligned}
& \left|\varphi \operatorname{Tr}_{s}\left[N \exp \left(-\mathcal{D}_{3, u, T}^{2}\right)\right]-G_{1, u, T}\right| \\
& \quad=\left|\frac{1}{2 \pi i} \psi_{u} \varphi \operatorname{Tr}_{s}\left[N \int_{\Delta \cup T^{2} \Delta} \frac{\exp \left(-u^{2} \lambda\right)}{\lambda-\mathcal{D}_{3,1, T}^{2}} d \lambda\right]\right|<C e^{-u^{2} c_{1}} .
\end{aligned}
$$

Par (1.18), (1.50) et (1.67), on a

$$
\begin{array}{r}
\lim _{T \rightarrow+\infty} \int_{1}^{+\infty} 2\left\{\varphi \operatorname{Tr}_{s}\left[N \exp \left(-\mathcal{D}_{3, u, T}^{2}\right)\right]-G_{1, u, T}\right\} \frac{d u}{u} \\
=\int_{1}^{+\infty} 2\left\{\varphi \operatorname{Tr}_{s}\left[N \exp \left(-\mathcal{D}_{1, u}^{2}\right)\right]-G_{1, u, \infty}\right\} \frac{d u}{u} .
\end{array}
$$

Par (1.64), pour $T \geq T_{0}$, on a

$$
\begin{gathered}
\int_{1}^{+\infty} 2\left\{G_{1, u, T}-\varphi \operatorname{Tr}_{s}\left[N \exp \left(-\nabla_{T}^{H(\mathcal{E}), 2}\right)\right]\right\} \frac{d u}{u}=\sum_{r=2}^{n} 2 \int_{T^{-(r-1)}}^{+\infty} F_{r, u, T} \frac{d u}{u} \\
+2 \int_{T^{-n+1}}^{+\infty}\left\{G_{n, u, T}-\varphi \operatorname{Tr}_{s}\left[N \exp \left(-\nabla_{T}^{H(\mathcal{E}), 2}\right)\right]\right\} \frac{d u}{u}
\end{gathered}
$$

On décompose chaque intégrale $\int_{T^{-(r-1)}}^{+\infty}$ sous la forme $\int_{T^{-(r-1)}}^{1}+\int_{1}^{+\infty}$. En sommant les termes $\int_{1}^{+\infty}$, on obtient un terme $Q_{1, T}$, et en sommant les termes $\int_{T^{-(r-1)}}^{1}$, on obtient un terme $Q_{2, T}$. Alors par la Proposition 1.10 et le théorème de convergence dominée, quand $T \rightarrow+\infty$, on a

$$
\begin{aligned}
Q_{1, T} \rightarrow Q_{1, \infty}= & \sum_{r=2}^{n} 2 \int_{1}^{+\infty} F_{r, u, \infty} \frac{d u}{u} \\
& +2 \int_{1}^{+\infty}\left(G_{n, u, \infty}-\operatorname{ch}^{\prime}\left(\mathcal{E}_{n+1}, h^{\mathcal{E}_{n+1}}\right)\right) \frac{d u}{u} .
\end{aligned}
$$

En utilisant la Proposition 1.10, et le théorème de convergence dominée, quand $T \rightarrow+\infty$, on a

$$
\begin{aligned}
& Q_{2, T}-2 \sum_{r=2}^{n+1}(r-1)\left[\operatorname{ch}^{\prime}\left(\mathcal{E}_{r}, h^{\mathcal{E}_{r}}\right)-\operatorname{ch}^{\prime}\left(\mathcal{E}_{r+1}, h^{\mathcal{E}_{r+1}}\right)\right] \log T \\
= & 2 \sum_{r=2}^{n} \int_{T^{-(r-1)}}^{1}\left(F_{r, u, T}-\Sigma_{i=-2 m}^{0} a_{r, i, T} u^{i}\right) \frac{d u}{u}+O\left(\frac{1}{T} \log T\right)
\end{aligned}
$$


FORMES DE TORSION ANALYTIQUE ET FAMILLES DE SUBMERSIONS II

$$
\begin{array}{r}
+2 \sum_{i=-2 m}^{-1} \frac{1}{i}\left[\left(b_{n, i, T}+\Sigma_{r=2}^{n} a_{r, i, T}\right)-\left(b_{n, i, T} T^{-(n-1) i}+\Sigma_{r=2}^{n} a_{r, i, T} T^{-(r-1) i}\right)\right] \\
\rightarrow Q_{2, \infty}=2 \sum_{r=2}^{n} \int_{0}^{1}\left(F_{r, u, \infty}-\Sigma_{i=-2 m}^{0} a_{r, i, \infty} u^{i}\right) \frac{d u}{u} \\
+2 \sum_{r=1}^{n-1} \sum_{i=-2 m}^{-1} \frac{1}{i} a_{r, i, \infty} .
\end{array}
$$

Par (1.48) et par la Proposition 1.10, on a

$$
\begin{aligned}
Q_{2, \infty}=2 \sum_{r=2}^{n} & \int_{0}^{1}\left\{\varphi \operatorname{Tr}_{s}\left[N \exp \left(-\mathcal{D}_{r, u}^{2}\right)\right]-\operatorname{ch}^{\prime}\left(\mathcal{E}_{r}, h^{\mathcal{E}_{r}}\right)\right\} \frac{d u}{u} \\
& +2 \sum_{r=1}^{n-1} \int_{1}^{+\infty}\left\{G_{r, u, \infty}-\operatorname{ch}^{\prime}\left(\mathcal{E}_{r+1}, h^{\mathcal{E}_{r+1}}\right)\right\} \frac{d u}{u}
\end{aligned}
$$

D'après (1.48), (1.68)-(1.72), on a le Théorème 1.6.

f) Preuve du Théorème 1.7. Soit $F=F^{0} \supset F^{1} \supset \cdots \supset F^{n}=0$ une filtration de fibrés vectoriels holomorphes de $F$ sur $S$. Pour $i \geq 0$, on pose $\operatorname{Gr}^{i} F=F^{i} / F^{i+1}$. Soit $h^{F}$ (resp $h^{\mathrm{Gr} F}$ ) une métrique hermitienne sur $F^{i}$ (resp. GrF). Soit $h^{F^{i}}$ la métrique sur $F^{i}$ induite par $h^{F}$.

Soit $G^{i}$ l'espace orthogonal à $F^{i+1}$ dans $F^{i}$. Alors $G^{i}$ est $\mathcal{C}^{\infty}$-isomorphe à $\operatorname{Gr}^{i} F$ sur $S$. Soit $h^{G^{i}}$ la métrique sur $G^{i}$ induite par $h^{G^{i} F}$. Soit $P^{G^{i}}$ la projection orthogonale de $F$ sur $G^{i}$. On note $N_{H}$ l'opérateur de nombre sur $G^{\bullet}$ et $\operatorname{Gr} F$. Soit $h^{{ }^{F}}=\oplus h^{G^{i}}$ la métrique sur $F=\oplus G^{i}$.

Soit $h_{T}^{F}(T \geq 1)$ une famille de métriques sur $F$ telle que $h_{1}^{F}=h^{F}$ et qu'il existe $\delta>0$, tel que quand $T \rightarrow+\infty$, pour $s_{1} \in F^{i}, s_{2} \in F^{j}$, on a

$$
\begin{aligned}
& \left\langle s_{1}, s_{2}\right\rangle_{h_{T}^{F}}=T^{2 n-i-j}\left(\left\langle P^{G^{i}} s_{1}, P^{G^{j}} s_{2}\right\rangle_{h^{\prime}}+O\left(\frac{1}{T^{\delta}}\right)\right) . \\
& \left(h_{T}^{F}\right)^{-1} \frac{\partial}{\partial T} h_{T}^{F}=\frac{1}{T} T^{N_{H}}\left(2\left(n-N_{H}\right)+O\left(\frac{1}{T^{\delta}}\right)\right) T^{-N_{H}} .
\end{aligned}
$$

Soient $\nabla_{T}^{F^{i}}, \nabla_{T}^{F}, \nabla^{\mathrm{Gr} F}$ les connexions holomorphes hermitiennes $\operatorname{sur}\left(F^{i}, h_{T}^{F^{i}}\right)$, $\left(F, h_{T}^{F}\right),\left(\mathrm{Gr} F, h^{\mathrm{Gr} F}\right)$.

Proposition 1.11. Quand $T \rightarrow+\infty$, on a

$$
\varphi \operatorname{Tr}\left[\left(h_{T}^{F}\right)^{-1} \frac{\partial}{\partial T} h_{T}^{F} \exp \left(-\nabla_{T}^{F, 2}\right)\right]=2 \varphi \operatorname{Tr}\left[\frac{n-N_{H}}{T} \exp \left(-\nabla^{\mathrm{Gr} F, 2}\right)\right]+O\left(\frac{1}{T^{1+\delta}}\right)
$$

Soit

$$
\begin{array}{r}
I\left(F, \mathrm{Gr} F, h_{T}^{F}, h^{\mathrm{Gr} F}\right)=-\int_{1}^{+\infty}\left\{\varphi \operatorname{Tr}\left[\left(h_{T}^{F}\right)^{-1} \frac{\partial}{\partial T} h_{T}^{F} \exp \left(-\nabla_{T}^{F, 2}\right)\right]\right. \\
\left.-2 \varphi \operatorname{Tr}\left[\frac{n-N_{H}}{T} \exp \left(-\nabla^{\mathrm{Gr} F, 2}\right)\right]\right\} d T .
\end{array}
$$

Alors on a

$$
I\left(F, \operatorname{Gr} F, h_{T}^{F}, h^{\mathrm{Gr} F}\right)=\tilde{\mathrm{ch}}\left(F, \mathrm{Gr} F, h^{F}, h^{\mathrm{Gr} F}\right) \quad \text { dans } \quad P^{S} / P^{S, 0}
$$


Preuve. i) Soit $\tau$ l'isomorphisme canonique de $G^{i}$ à $\mathrm{Gr}^{i} F$. Soit $\nabla^{G^{i}}=\tau^{*} \nabla^{\mathrm{Gr}}{ }^{i} F$. Soit $A_{i} \in T^{*(0,1)} S \otimes \operatorname{Hom}\left(F^{i}, F^{i+1}\right)$ tel que

$$
\nabla^{F^{i \prime \prime}}=\nabla^{G^{i \prime \prime}}+\nabla^{F^{i+1} \prime \prime}+A_{i}
$$

On pose

$$
\nabla_{\infty}^{F}=\oplus_{i=0}^{n}\left(\nabla^{G^{i}}+A_{i}\right)
$$

Alors on vérifie facilement que quand $T \rightarrow+\infty$, on a

$$
\nabla_{T}^{F^{i}}=\nabla^{G^{i}} \oplus \nabla_{T}^{F^{i+1}}+A_{i}+O\left(\frac{1}{T^{\delta}}\right)
$$

et donc

$$
\nabla_{T}^{F}=\nabla_{\infty}^{F}+O\left(\frac{1}{T^{\delta}}\right)
$$

Si l'on écrit $\nabla_{\infty}^{F, 2}$ sous forme matricielle relativement au scindage $F=\oplus G^{i}$, alors on obtient une matrice triangulaire.

En utilisant (1.73) et (1.80), on a (1.74).

D'après [BGS1, Théorème 1.24], (1.74), (1.75) et (1.80), on a

$$
\begin{gathered}
\frac{\bar{\partial} \partial}{2 i \pi} I\left(F, \mathrm{Gr} F, h_{T}^{F}, h^{\mathrm{Gr} F}\right)=\int_{1}^{+\infty} \frac{\partial}{\partial T} \varphi \operatorname{Tr}\left[\exp \left(-\nabla_{T}^{F, 2}\right)\right] d T \\
=-\operatorname{ch}\left(F, h^{F}\right)+\operatorname{ch}\left(\mathrm{Gr} F, h^{\mathrm{Gr} F}\right) .
\end{gathered}
$$

ii) Soit $\mathbf{P}^{1}$ le plan projectif complexe. Soit $z$ le paramètre complexe standard sur $\mathbf{P}^{1}$, et $i_{z}: S \rightarrow S \times \mathbf{P}^{1}$ l'application de $x \in S$ à $(x, z) \in S \times \mathbf{P}^{1}$. Alors comme dans [BGS1, Théorème 1.29] ou dans [Ma1, $\S 11(\mathrm{~d})]$, on peut construire des fibrés vectoriels holomorphes $\tilde{F}^{j}$ et $\widehat{\mathrm{Gr}^{j} F}$ sur $S \times \mathbf{P}^{1}$ tels qu'on a les suites exactes

$$
0 \rightarrow \widetilde{F}^{j+1} \rightarrow \widetilde{F}^{j} \rightarrow \widetilde{\mathrm{Gr}^{j} F} \rightarrow 0 \text {. }
$$

De plus, si on note $\widetilde{F}=\widetilde{F}^{0}$, alors $i_{0}^{*} \widetilde{F}=F, i_{\infty}^{*} \widetilde{F}=\mathrm{Gr} F$.

Par partition de l'unité, on peut trouver une famille de métriques $h_{T}^{\widetilde{F}}$ (resp. $h^{\widetilde{\mathrm{Gr} F}}$ ) sur $\widetilde{F}$ (resp. $\widetilde{\mathrm{Gr} F}$ ) telle qu'elle vérifie (1.73), et que sous l'identification ci-dessus,

$$
h_{T}^{i_{0}^{*} \tilde{F}}=h_{T}^{F}, \quad h_{T}^{i_{\infty}^{*} \widetilde{F}}=\oplus T^{2 n-2 i} h^{\mathrm{Gr}^{i} F}, \quad h^{i_{0}^{*} \widetilde{\mathrm{Gr} F}}=h_{\infty}^{i_{\infty}^{*} \widetilde{\mathrm{Gr} F}}=h^{\mathrm{Gr} F} .
$$

Alors d'après i), le terme $I\left(\widetilde{F}, \widetilde{\mathrm{Gr} F}, h_{T}^{\widetilde{F}}, h^{\widetilde{\mathrm{Gr} F}}\right)$ est bien défini. Par la preuve de [BGS1, Théorème 1.29], on a

$$
\widetilde{\operatorname{ch}}\left(F, \operatorname{Gr} F, h^{F}, h^{\mathrm{Gr} F}\right)=-\int_{\mathbf{P}^{1}} \operatorname{ch}\left(\widetilde{F}, h_{1}^{\widetilde{F}}\right) \log |z|^{2} .
$$

Comme les identifications $i_{0}^{*} \widetilde{\mathrm{Gr} F} \simeq i_{\infty}^{*} \widetilde{\mathrm{Gr} F} \simeq \mathrm{Gr} F$ sont des isométries, on a

$$
\int_{\mathbf{P}^{1}} \operatorname{ch}\left(\widetilde{\mathrm{Gr} F}, h^{\widetilde{\mathrm{Gr} F}}\right) \log |z|^{2}=-\widetilde{\operatorname{ch}}\left(\mathrm{Gr} F, h^{\mathrm{Gr} F}, h^{\mathrm{Gr} F}\right)=0 \quad \text { dans } P^{S} / P^{S, 0}
$$


D'après (1.81), (1.84) et (1.85), on a

$$
\begin{array}{r}
\tilde{\operatorname{ch}}\left(F, \operatorname{Gr} F, h^{F}, h^{\mathrm{Gr} F}\right)=\int_{\mathbf{P}^{1}} \frac{\bar{\partial} \partial}{2 i \pi} I\left(\widetilde{F}, \widetilde{\mathrm{Gr} F}, h_{T}^{\widetilde{F}}, h^{\widetilde{\mathrm{Gr} F}}\right) \log |z|^{2} \\
=\int_{\mathbf{P}^{1}} I\left(\widetilde{F}, \widetilde{\mathrm{Gr} F}, h_{T}^{\widetilde{F}}, h^{\widetilde{\mathrm{Gr} F}}\right) \frac{\bar{\partial} \partial}{2 i \pi} \log |z|^{2} \quad \text { dans } P^{S} / P^{S, 0} .
\end{array}
$$

Par [Ma1, (11.17)], (1.83) et (1.86), on a

$$
\begin{array}{r}
\tilde{\operatorname{ch}}\left(F, \operatorname{Gr} F, h^{F}, h^{\mathrm{Gr} F}\right)=i_{0}^{*} I\left(\widetilde{F}, \widetilde{\mathrm{Gr} F}, h_{T}^{\widetilde{F}}, h^{\widetilde{\mathrm{Gr} F}}\right)-i_{\infty}^{*} I\left(\widetilde{F}, \widetilde{\mathrm{Gr} F}, h_{T}^{\widetilde{F}}, h^{\widetilde{\mathrm{Gr} F}}\right) \\
=I\left(F, \mathrm{Gr} F, h_{T}^{F}, h^{\mathrm{Gr} F}\right)-I\left(i_{\infty}^{*} \widetilde{F}, i_{\infty}^{*} \widetilde{\mathrm{Gr} F}, h_{T}^{i_{\infty}^{*} \widetilde{F}}, h_{\infty}^{i_{\infty}^{*} \widetilde{\mathrm{Gr} F}}\right) \\
\operatorname{dans} P^{S} / P^{S, 0}
\end{array}
$$

En utilisant (1.75) et (1.83), on sait que

$$
I\left(i_{\infty}^{*} \widetilde{F}, i_{\infty}^{*} \widetilde{\mathrm{Gr} F}, h_{T}^{i_{\infty}^{*} \widetilde{F}}, h_{\infty}^{i_{\infty}^{*} \widetilde{\mathrm{Gr} F}}\right)=0 \quad \text { dans } \quad P^{S} / P^{S, 0} .
$$

Par (1.87) et (1.88), on a (1.76).

PREUVE du Théorème 1.7: On rappelle que $P_{T}$ est la projection orthogonale de $\mathcal{E}$ sur $\operatorname{Ker} D_{T}^{\prime}$ associée à $h_{T}^{\mathcal{E}}$. Par la théorie de Hodge, l'application $s \in \operatorname{Ker} D_{1}^{\prime} \rightarrow$ $P_{T} s \in \operatorname{Ker} D_{T}^{\prime}$ est l'identification canonique. Soit $\widetilde{P}_{T}$ la projection orthogonale de $\mathcal{E}$ $\operatorname{sur} \widetilde{\mathcal{E}}_{T}=\operatorname{Ker} A_{T}$ associée à $h^{\mathcal{E}}$. Comme en [BerB, (6.61)], on a

$$
P_{T}=T^{-N_{V}} \widetilde{P}_{T} T^{N_{V}} .
$$

D'après (1.30), on a aussi

$$
\widetilde{P}_{T}=p_{n+1, T}
$$

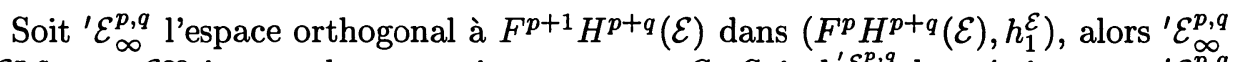
et $\mathcal{E}_{\infty}^{p, q}$ sont $\mathcal{C}^{\infty}$ isomorphes canoniquement sur $S$. Soit $h^{\prime} \mathcal{E}_{\infty}^{p, q}$ la métrique sur ${ }^{\prime} \mathcal{E}_{\infty}^{p, q}$ induite par $h^{\mathcal{E}_{\infty}^{p, q}}$, soit $h^{\prime H(\mathcal{E})}=\oplus h^{\prime} \mathcal{E}_{\infty}^{p, q}$ la métrique sur $H(\mathcal{E})=\oplus^{\prime} \mathcal{E}_{\infty}^{p, q}$. Soit $P_{p, q, \infty}$ la projection de $H(\mathcal{E})=\oplus^{\prime} \mathcal{E}_{\infty}^{p, q} \operatorname{sur}^{\prime} \mathcal{E}_{\infty}^{p, q}$.

D'après (1.90), pour $\alpha \in F^{p_{1}} H^{q}(\mathcal{E}), \beta \in F^{p_{2}} H^{q}(\mathcal{E})$, quand $T \rightarrow+\infty$,

$$
\begin{aligned}
& \langle\alpha, \beta\rangle_{h_{T}^{H(\varepsilon)}}=\left\langle P_{T} \alpha, P_{T} \beta\right\rangle_{h_{T}^{\mathcal{E}}} \\
& \quad=T^{2 q-p_{1}-p_{2}}\left[\left\langle P_{p_{1}, q-p_{1}, \infty} \alpha, P_{p_{2}, q-p_{2}, \infty} \beta\right\rangle_{h^{\prime H(\varepsilon)}}+O\left(\frac{1}{T}\right)\right] .
\end{aligned}
$$

Comme dans [B4, (6.45)-(6.48)], on sait que

$$
\left(h_{T}^{H(\mathcal{E})}\right)^{-1} \frac{\partial}{\partial T} h_{T}^{H(\mathcal{E})}=\frac{2}{T} P_{T} N_{V} P_{T}=\frac{2}{T} T^{-N_{V}} \widetilde{P}_{T} N_{V} \widetilde{P}_{T} T^{N_{V}}
$$

Par (1.31), (1.90) et (1.92), quand $T \rightarrow+\infty$, on a

$$
\left(h_{T}^{H(\mathcal{E})}\right)^{-1} \frac{\partial}{\partial T} h_{T}^{H(\mathcal{E})}=\frac{2}{T} T^{-N_{V}}\left(N_{V}+O\left(\frac{1}{T}\right)\right) T^{N_{V}} .
$$

En utilisant la Proposition 1.11, (1.91) et (1.93), on a le Théorème 1.7. 
2. Fonctorialité des formes de torsion analytique. Soit $\pi_{1}: W \rightarrow V$, $\pi_{2}: V \rightarrow S$ des submersions holomorphes de variétés complexes de fibres compactes $X, Y$. Alors $\pi_{3}=\pi_{2} \circ \pi_{1}: W \rightarrow S$ est une submersion holomorphe de fibre compacte $Z$. Soit $\xi$ un fibré holomorphe sur $W$. Soit $\left(E_{r, s}, d_{r, s}\right)(r \geq 2, s \in S)$ la suite spectrale de Leray associée à la fibration $Z_{s} \rightarrow Y_{s}$. Dans cette Section, on établit la fonctorialité des formes de torsion analytique énoncée au Théorème 0.1 , dans le cas où le rang des fibres $E_{r}$ est localement constant sur $S$. Ce résultat est compatible à [Ma1, Théorème 3.11] quand $\pi_{1}$ et $V$ sont projectives. Notre résultat est plus général que celui que nous avons obtenu dans [Ma1, §4], où nous supposons que les $H^{i}$ sont nuls pour $i>0$.

On définit en particulier la classe de Bott-Chern $\widetilde{\operatorname{ch}}\left(E_{2}, H\left(Z, \xi_{\mid Z}\right), h^{E_{2}}, h^{H\left(Z, \xi_{\mid Z}\right)}\right)$ $\in P^{S} / P^{S, 0}$. On montre que quand $\pi_{1}$ et $V$ sont projectives, cette nouvelle définition coïncide avec la Définition de $\tilde{\operatorname{ch}}\left(E_{2}, H\left(Z, \xi_{\mid Z}\right), h^{E_{2}}, h^{H\left(Z, \xi_{\mid Z}\right)}\right)$ donnée dans [Ma1, (10.29)]. On utilise pour cela, de manière essentielle, le Théorème 1.2.

Pour démontrer le Théorème 0.1 , on procède comme dans [Ma1, §4]. Par rapport à $[\mathrm{Ma} 1, \S 4]$, on a une difficulté supplémentaire, puisque la suite spectrale ne dégénère en général pas en $E_{2}$. Pour résoudre cette difficulté, on procède comme à la Section 1 , où nous avons considéré un problème comparable en dimension finie, en décomposant les supertraces du noyau de chaleur d'une superconnexion qui dépend de deux paramètres $u$ et $T$, suivant le comportement asymptotique des valeurs propres de $A_{T}^{2}$ quand $T \rightarrow+\infty$. Pour calculer l'asymptotique de ces supertraces quand $T \rightarrow+\infty$ ou $u \rightarrow 0$, les résultats de [BerB, §6] ne sont pas suffisants, et on procède comme dans [Ma1, §5].

Cette Section est organisée de la façon suivante. Dans (a), on montre que le complexe de Dolbeault muni d'une filtration convenable calcule la suite spectrale de Leray au sens de Grothendieck [Grot]. Dans (b), on définit la classe de Bott-Chern $\tilde{\mathrm{ch}}\left(E_{2}, H\left(Z, \xi_{\mid Z}\right), h^{E_{2}}, h^{H\left(Z, \xi_{\mid Z}\right)}\right)$ dans le cas où le rang des fibres $E_{r}$ est localement constant sur $S$, et on reénonce le Théorème 0.1 comme le Théorème 2.6. Dans (c), on énonce des résultats intermédiaires qui sont utilisés dans la preuve du Théorème 2.6. Dans (d), on montre le Théorème 2.6. Dans (e), on étudie l'asymptotique de certaines supertraces quand $T \rightarrow+\infty$ ou $u \rightarrow 0$. Dans (f) et (g), on démontre les résultats intermédiaires.

Dans cette Section, on utilise les mêmes notations qu'à [Ma1, §3, 4, 5, 10(a)].

a) Complexe de Dolbeault. Soient $Z$ et $Y$ des variétés complexes. Soit $\pi: Z \rightarrow Y$ une submersion holomorphe de fibre compacte $X$. Soit $\xi$ un fibré vectoriel holomorphe sur $Z$. Soit $\left(E_{r}, d_{r}\right)(r \geq 2)$ la suite spectrale de Leray correspondant à $(\pi, \xi)$, définie comme dans [Ma1, 10(b)].

Soit

$$
\begin{array}{r}
\Lambda^{\bullet}\left(T^{*(0,1)} Z\right)=F^{0}\left(\Lambda^{\bullet}\left(T^{*(0,1)} Z\right)\right) \supset F^{1}\left(\Lambda^{\bullet}\left(T^{*(0,1)} Z\right)\right) \supset \cdots \\
\supset F^{\operatorname{dim} Y+1}\left(\Lambda^{\bullet}\left(T^{*(0,1)} Z\right)\right)=\{0\} .
\end{array}
$$

la filtration standard de $\Lambda^{\bullet}\left(T^{*(0,1)} Z\right)$ : si $0 \leq p \leq q \leq \operatorname{dim} Z$, alors $\alpha \in F^{p} \Lambda^{q}\left(T^{*(0,1)} Z\right)$ si et seulement si

$$
X_{1}, \cdots, X_{q-p+1} \in \overline{T X}, \quad \text { alors } \quad i_{X_{1}} \cdots i_{X_{q-p+1}} \alpha=0 .
$$

Cette filtration induit une filtration sur le complexe de Dolbeault $\left(\Omega(Z, \xi), \bar{\partial}^{Z}\right)$. Soit $\left(E_{r}^{\prime}, d_{r}^{\prime}\right)$ la suite spectrale correspondante.

Par [BerB, (1.11)] et [Ma1,(10.24)], on a un isomorphisme naturel

$$
E_{2}^{\prime} \simeq H\left(Y, R^{\bullet} \pi_{*} \xi\right)=E_{2} .
$$


THÉORÈME 2.1. La suite spectrale $\left(E_{r}^{\prime}, d_{r}^{\prime}\right)(r \geq 2)$ est canoniquement isomorphe $\grave{a}$ la suite spectrale $\left(E_{r}, d_{r}\right)$ par un isomorphisme compatible à (2.2).

PREUVE. Le reste de cette sous-section est consacré à la preuve du Théorème 2.1 .

Soit $\mathcal{O}_{Z}^{\infty}, \mathcal{O}_{Y}^{\infty}$ les faisceaux des fonctions $\mathcal{C}^{\infty} \operatorname{sur} Z, Y$, et soit $\mathcal{D}_{Z}^{\bullet}, \mathcal{D}_{Y}^{\bullet}$ les $\mathcal{O}_{Z}^{\infty}$, $\mathcal{O}_{Y}^{\infty}$ faisceaux associés aux complexes de Dolbeault sur $Z, Y$. Pour $p \geq 0, \mathcal{D}_{Z}^{p}$ est le faisceau des sections $\mathcal{C}^{\infty}$ de $\Lambda^{p}\left(T^{*(0,1)} Z\right)$, la différentielle $\bar{\partial}^{Z}$ est $\mathcal{O}_{Z}$-linéaire. Par [Go, II 3.7], si $G$ est un $\mathcal{O}_{Z}$-faisceau, le complexe

$$
\mathcal{D}_{Z}^{\bullet}(G)=G \otimes_{\mathcal{O}_{Z}} \mathcal{D}_{Z}^{\bullet}
$$

est une résolution de $G$, car pour $p \geq 0, \mathcal{D}_{Z}^{p}$ est un $\mathcal{O}_{Z}$-faisceau plat [M]. De plus $\mathcal{D}_{Z}^{p}(G)$ est fin [Go, Théorème 3.7.3]. D'où

$$
R^{p} \pi_{*} G=H^{p}\left(\pi_{*} \mathcal{D}_{Z}^{\bullet}(G)\right)
$$

Soit $\mathcal{D}_{X}^{\bullet}$ le complexe de Dolbeault relatif dont $\mathcal{D}_{X}^{p}$ est le faisceau des sections $\mathcal{C}^{\infty}$ de $\Lambda^{p}\left(T^{*(0,1)} X\right)$ sur $Z$, et dont la différentielle $\bar{\partial}^{X}$ est l'opérateur $\bar{\partial}$ le long des fibres. On remarque que $\bar{\partial}^{X}$ est $\mathcal{O}_{Z}$ et $\mathcal{O}_{Y}^{\infty}$-linéaire.

Dans la suite, on note $F$ le $\mathcal{O}_{Z}$-faisceau $\mathcal{O}_{Z}(\xi)$, et $\left(\mathcal{F}_{0}, d\right)$ le complexe $\left(\mathcal{D}_{Z}^{\bullet}(F), \bar{\partial}^{Z}\right)$.

Soit $\mathcal{F}_{1}=\left(\mathcal{F}_{1}^{p, q}\right)$ le bicomplexe de faisceaux sur $Y$ défini par

$$
\mathcal{F}_{1}^{p, q}=\pi_{*} \mathcal{D}_{Z}^{p}(F) \otimes_{\mathcal{O}_{Y}} \mathcal{D}_{Y}^{q}
$$

dont les différentielles sont $1 \otimes \bar{\partial}^{Y}: \mathcal{F}_{1}^{p, q} \rightarrow \mathcal{F}_{1}^{p, q+1}$ et $\bar{\partial}^{Z} \otimes 1: \mathcal{F}_{1}^{p, q} \rightarrow \mathcal{F}_{1}^{p+1, q}$. Par le même argument que précédemment, on sait que le bicomplexe $\mathcal{F}_{1}$ est une résolution acyclique du complexe de $\mathcal{O}_{Y}$-faisceaux $\pi_{*} \mathcal{D}_{Z}^{\bullet}(F)$.

On filtre le complexe $E\left(\mathcal{F}_{1}\right)=\left(\Gamma_{Y}\left(\mathcal{F}_{1}\right), \bar{\partial}^{Z} \otimes 1+1 \otimes \bar{\partial}^{Y}\right)$ par $F^{p}\left(\Gamma_{Y}\left(\mathcal{F}_{1}\right)\right)=$ $\oplus_{p^{\prime} \geq p} \Gamma_{Y}\left(\mathcal{F}_{1}^{\bullet, p^{\prime}}\right)$. Alors d'après [Ma1, Remarque 10.12], la suite spectrale $\left(E_{r}\left(\mathcal{F}_{1}\right), d_{r}\right)$ associée à $\Gamma_{Y}\left(\mathcal{F}_{1}\right)$ calcule la suite spectrale de Leray (à partir de $r=2$ ).

Soit $\mathcal{F}_{2}=\left(\mathcal{F}_{2}^{p, q}\right)$ le bicomplexe de faisceaux sur $Z$ défini par

$$
\mathcal{F}_{2}^{p, q}=\mathcal{D}_{Z}^{p}(F) \otimes_{\mathcal{O}_{Z}} \mathcal{D}_{Z}^{q}
$$

dont les différentielles sont $1 \otimes \bar{\partial}^{Z}: \mathcal{F}_{2}^{p, q} \rightarrow \mathcal{F}_{2}^{p, q+1}$ et $\bar{\partial}^{Z} \otimes 1: \mathcal{F}_{2}^{p, q} \rightarrow \mathcal{F}_{2}^{p+1, q}$. On note $\left(\mathcal{F}_{2}, d\right)$ le complexe total correspondant.

La filtration (2.1) induit une filtration du complexe de $\mathcal{O}_{Z}$-faisceaux $\left(\mathcal{D}_{Z}^{\bullet}, \bar{\partial}^{Z}\right)$. On a donc une filtration du complexe $\left(\mathcal{F}_{2}, d\right)$

$$
\begin{aligned}
\left(\mathcal{F}_{2}, d\right)=\left(F^{0} \mathcal{F}_{2}, d\right) \supset\left(F^{1} \mathcal{F}_{2}, d\right)= & \left(\mathcal{D}_{Z}^{\bullet}(F) \otimes_{\mathcal{O}_{Z}} F^{1} \mathcal{D}_{Z}^{\bullet}\right) \supset \cdots \\
& \supset\left(F^{\operatorname{dim} Y+1} \mathcal{F}_{2}, d\right)=\{0\} .
\end{aligned}
$$

De même on a une filtration sur le complexe $\left(\mathcal{D}_{Z}^{\bullet}(F), \bar{\partial}^{Z}\right)=\left(\mathcal{F}_{0}, d\right)$.

Soit $j_{0}$ l'inclusion du complexe $\left(\mathcal{F}_{0}, d\right)$ dans le complexe $\left(\mathcal{F}_{2}, d\right)$ induite par l'inclusion $j_{0}: F \hookrightarrow \mathcal{D}_{Z}^{\bullet}(F)$ qu'on tensorise par $\mathcal{D}_{Z}^{\bullet}$. Alors $j_{0}$ induit une injection du complexe filtré $E\left(\mathcal{F}_{0}\right)=\left(\Gamma_{Z}\left(\mathcal{F}_{0}\right), d\right)$ dans le complexe filtré $E\left(\mathcal{F}_{2}\right)=\left(\Gamma_{Z}\left(\mathcal{F}_{2}\right), d\right)$

Pour $p, q \geq 0$, on a aussi l'application canonique

$$
\begin{aligned}
\pi_{*} \mathcal{D}_{Z}^{p}(F) \otimes \mathcal{O}_{Y} \mathcal{D}_{Y}^{q} \rightarrow \pi_{*} \mathcal{D}_{Z}^{p}(F) \otimes \mathcal{O}_{Y} \pi_{*} \pi^{*} \mathcal{D}_{Y}^{q} & \rightarrow \pi_{*}\left(\mathcal{D}_{Z}^{p}(F) \otimes \mathcal{O}_{Z} \pi^{*} \mathcal{D}_{Y}^{q}\right) \\
& \rightarrow \pi_{*}\left(\mathcal{D}_{Z}^{p}(F) \otimes_{\mathcal{O}_{Z}} \mathcal{D}_{Z}^{q}\right)
\end{aligned}
$$


Elle induit une application de complexes filtrés $j_{1}: E\left(\mathcal{F}_{1}\right) \rightarrow E\left(\mathcal{F}_{2}\right)$.

Si on note $\left(E_{r}\left(\mathcal{F}_{i}\right), d_{r}\right)(i=0,2)$ la suite spectrale correspondante à $\left(E\left(\mathcal{F}_{i}\right), d\right)$ filtré comme ci-dessus, alors pour $r \geq 0, j_{i}(i=0,1)$ induit une application de complexes $j_{i}: E_{r}\left(\mathcal{F}_{i}\right) \rightarrow E_{r}\left(\mathcal{F}_{2}\right)$. On a aussi

$$
\left(E_{r}\left(\mathcal{F}_{0}\right), d_{r}\right)=\left(E_{r}^{\prime}, d_{r}^{\prime}\right) .
$$

Dans la suite, on va montrer que pour $r=1$, les $j_{i}(i=0,1)$ sont des isomorphismes de complexes.

On calcule tout d'abord le terme $E_{0}\left(\mathcal{F}_{i}\right)(i=0,1,2)$.

On note $\mathcal{E}\left(\mathcal{F}_{0}\right), \mathcal{E}\left(\mathcal{F}_{1}\right), \mathcal{E}\left(\mathcal{F}_{2}\right)$ les complexes $\left(\pi_{*}\left(\mathcal{D}_{X}^{\bullet}(F)\right), \bar{\partial}^{X}\right),\left(\pi_{*}\left(\mathcal{D}_{Z}^{\bullet}(F)\right) \otimes_{\mathcal{O}_{Y}}\right.$ $\left.\mathcal{O}_{Y}^{\infty}, \bar{\partial}^{Z} \otimes 1\right),\left(\pi_{*}\left(\mathcal{D}_{Z}^{\bullet}(F) \otimes \mathcal{O}_{z} \mathcal{D}_{X}^{\bullet}\right), \bar{\partial}^{Z} \otimes 1+1 \otimes \bar{\partial}^{X}\right)$. Soit $H\left(\mathcal{E}\left(\mathcal{F}_{i}\right)\right)$ les cohomologies associées.

Pour $p, q \geq 0$, on a la suite exacte suivante

$$
0 \rightarrow F^{p+1} \mathcal{D}_{Z}^{p+q} \rightarrow F^{p} \mathcal{D}_{Z}^{p+q} \rightarrow \mathcal{D}_{X}^{q} \otimes_{\pi^{-1} \mathcal{O}_{Y}^{\infty}} \pi^{-1} \mathcal{D}_{Y}^{p} \rightarrow 0 .
$$

Comme $\mathcal{O}_{Z}^{\infty}$ est un $\mathcal{O}_{Z}$-faisceau plat, et comme $\mathcal{D}_{Z}^{\bullet}(F)$ est un $\mathcal{O}_{Z}^{\infty}$-faisceau localement libre de type fini, d'après (2.10), on a aussi les suites exactes :

$0 \rightarrow F \otimes_{\mathcal{O}_{Z}} F^{p+1} \mathcal{D}_{Z}^{p+q} \rightarrow F \otimes_{\mathcal{O}_{Z}} F^{p} \mathcal{D}_{Z}^{p+q} \rightarrow F \otimes_{\mathcal{O}_{Z}}\left(\mathcal{D}_{X}^{q} \otimes_{\pi^{-1} \mathcal{O}_{Y}^{\infty}} \pi^{-1} \mathcal{D}_{Y}^{p}\right) \rightarrow 0$.

$$
\begin{aligned}
0 \rightarrow & \mathcal{D}_{Z}^{\bullet}(F) \otimes_{\mathcal{O}_{Z}} F^{p+1} \mathcal{D}_{Z}^{p+q} \rightarrow \mathcal{D}_{Z}^{\bullet}(F) \otimes_{\mathcal{O}_{Z}} F^{p} \mathcal{D}_{Z}^{p+q} \\
& \rightarrow \mathcal{D}_{Z}^{\bullet}(F) \otimes_{\mathcal{O}_{Z}}\left(\mathcal{D}_{X}^{q} \otimes_{\pi^{-1} \mathcal{O}_{Y}^{\infty}} \pi^{-1} \mathcal{D}_{Y}^{p}\right) \rightarrow 0
\end{aligned}
$$

Comme $\mathcal{D}_{Z}^{\bullet}(F) \otimes_{\mathcal{O}_{Z}} F^{p} \mathcal{D}_{Z}^{p+q}, F \otimes_{\mathcal{O}_{Z}} F^{p} \mathcal{D}_{Z}^{p+q}, \mathcal{D}_{X}^{q}$ sont des faisceaux fins, et comme $\mathcal{D}_{Y}^{p}$ est un $\mathcal{O}_{Y}^{\infty}$-faisceau localement libre, $\operatorname{par}(2.11)$, on sait que

$$
E_{0}^{p, q}\left(\mathcal{F}_{i}\right)=\Gamma_{Y}\left(\mathcal{E}^{q}\left(\mathcal{F}_{i}\right) \otimes_{\mathcal{O}_{Y}^{\infty}} \mathcal{D}_{Y}^{p}\right) .
$$

Comme $\mathcal{D}_{Y}^{p}$ est un $\mathcal{O}_{Y}^{\infty}$-faisceau localement libre et fin, d'après (2.12), on a

$$
E_{1}^{p, q}\left(\mathcal{F}_{i}\right)=\Gamma_{Y}\left(H^{q}\left(\mathcal{E}\left(\mathcal{F}_{i}\right)\right) \otimes_{\mathcal{O}_{Y}^{\infty}} \mathcal{D}_{Y}^{p}\right) .
$$

Dans (2.8), pour $q=0$, on obtient une application de complexes $j_{1}: \mathcal{E}\left(\mathcal{F}_{1}\right) \rightarrow$ $\mathcal{E}\left(\mathcal{F}_{2}\right)$. Soit $j_{0}$ l'inclusion du complexe $\mathcal{E}\left(\mathcal{F}_{0}\right)$ dans le complexe $\mathcal{E}\left(\mathcal{F}_{2}\right)$, induite par l'inclusion $j_{0}: F \hookrightarrow \mathcal{D}_{Z}^{\circ}(\mathcal{F})$ qu'on tensorise par $\mathcal{D}_{X}^{\bullet}$. Par (2.12) et (2.13), l'application $j_{i}: E_{0}\left(\mathcal{F}_{i}\right) \rightarrow E_{0}\left(\mathcal{F}_{2}\right)$ est induite par $j_{i}: \mathcal{E}\left(\mathcal{F}_{i}\right) \rightarrow \mathcal{E}\left(\mathcal{F}_{2}\right)$, et l'application $j_{i}$ : $E_{1}\left(\mathcal{F}_{i}\right) \rightarrow E_{1}\left(\mathcal{F}_{2}\right)$ est induite par $j_{i}: H\left(\mathcal{E}\left(\mathcal{F}_{i}\right)\right) \rightarrow H\left(\mathcal{E}\left(\mathcal{F}_{2}\right)\right)$.

Lemme 2.2. Soit $G$ un $\mathcal{O}_{Z}$-faisceau plat et fin sur $Z$, et soit $R$ un $\mathcal{O}_{Y}$-faisceau. Alors il existe un isomorphisme canonique

$$
\pi_{*} G \otimes \mathcal{O}_{Y} R \simeq \pi_{*}\left(G \otimes \mathcal{O}_{Z} \pi^{*} R\right) .
$$

PrEUVE. En effet, on a les morphismes canoniques

$$
\pi_{*} G \otimes \mathcal{O}_{Y} R \rightarrow \pi_{*} G \otimes_{\mathcal{O}_{Y}} \pi_{*} \pi^{*} R \rightarrow \pi_{*}\left(G \otimes_{\mathcal{O}_{Z}} \pi^{*} R\right) .
$$

On note $\tau: \pi_{*} G \otimes_{\mathcal{O}_{Y}} R \rightarrow \pi_{*}\left(G \otimes_{\mathcal{O}_{z}} \pi^{*} R\right)$ le morphisme composé. Pour montrer le Lemme, il suffit de montrer que pour tout $x \in Y$,

$$
\tau_{x}:\left(\pi_{*} G\right)_{x} \otimes_{\mathcal{O}_{Y, x}} R_{x}=\left(\pi_{*} G \otimes_{\mathcal{O}_{Y}} R\right)_{x} \rightarrow \pi_{*}\left(G \otimes_{\mathcal{O}_{Z}} \pi^{*} R\right)_{x}
$$


est un isomorphisme.

i) On suppose que $R$ est un $\mathcal{O}_{Y}$-faisceau cohérent.

Soit $x \in Y$, alors il existe $x \in U$ un ouvert de $Y$ et $L^{i}$ des $\mathcal{O}_{Y}$-faisceaux libres de rang fini sur $U$, tels que $\left(L^{i}\right)_{0 \leq i \leq n}$ est une résolution projective de $R$ sur $U$. Comme $\pi$ est plat, on sait que $\left(\pi^{*}\left(L^{i}\right)\right)$ est aussi une résolution projective de $\pi^{*} R$ sur $\pi^{-1}(U)$. Donc on a le diagramme commutatif suivant:

$$
\begin{aligned}
& 0 \rightarrow \pi_{*}\left(G \otimes \mathcal{O}_{Z} \pi^{*} L^{n}\right) \rightarrow \cdots \rightarrow \pi_{*}\left(G \otimes \mathcal{O}_{Z} \pi^{*} L^{0}\right) \rightarrow \quad \pi_{*}\left(G \otimes \mathcal{O}_{Z} \pi^{*} R\right) \quad \rightarrow 0 \\
& 0 \rightarrow \quad \pi_{*} G \stackrel{\uparrow}{\otimes_{\mathcal{O}_{Y}}} L^{n} \rightarrow \quad \cdots \rightarrow \quad \pi_{*} G \stackrel{\uparrow}{\otimes_{\mathcal{O}_{Y}}} L^{0} \rightarrow \quad \pi_{*} G \stackrel{\uparrow}{\otimes_{\mathcal{O}_{Y}}} R \quad \rightarrow 0 .
\end{aligned}
$$

En utilisant que $G$ est un $\mathcal{O}_{Z}$-faisceau plat et fin, on voit que la première ligne de (2.15) est exacte. D'après [H, Exercice 2.2.1], on a aussi

$$
\pi_{*}\left(G \otimes_{\mathcal{O}_{Z}} \pi^{*} L^{i}\right) \simeq \pi_{*} G \otimes_{\mathcal{O}_{Y}} L^{i}
$$

On sait aussi que le foncteur $\otimes$ est exact à droite, d'où la deuxième ligne de (2.15) est exacte. Par un argument de suite spectrale, on a (2.14).

ii) Injectivité. Soit $f \in \operatorname{Ker} \tau_{x}(x \in Y)$, alors, il existe $g_{i} \in\left(\pi_{*} G\right)_{x}, r_{i} \in R_{x}(i=$ $1, \cdots, k)$ tels que $f=\Sigma_{i=1}^{k} g_{i} \otimes r_{i}$. On note $R^{\prime}$ le sous faisceau de $R$ engendré par $r_{i}$ dans un voisinage $U$ de $x$. Alors $R^{\prime}$ est un $\mathcal{O}_{Y}$-faisceau cohérent. Comme $G$ est plat, l'application $G \otimes \mathcal{O}_{z} \pi^{*} R^{\prime} \rightarrow G \otimes_{\mathcal{O}_{z}} \pi^{*} R$ est injective. Donc l'application $\pi_{*}\left(G \otimes_{\mathcal{O}_{z}} \pi^{*} R^{\prime}\right) \rightarrow \pi_{*}\left(G \otimes_{\mathcal{O}_{z}} \pi^{*} R\right)$ est aussi injective. Comme $f \in\left(\pi_{*} G \otimes_{\mathcal{O}_{z}} R^{\prime}\right)_{x}$, par i), on sait que $f=0$.

iii) Surjectivité. Si $f \in \pi_{*}\left(G \otimes_{\mathcal{O}_{z}} \pi^{*} R\right)_{x}(x \in Y)$, alors il existe un voisinage $U$ de $x$, tel que $f$ est une section de $G \otimes \mathcal{O}_{z} \pi^{*} R$ sur $\pi^{-1}(U)$. Donc pour tout $y \in \pi^{-1}(x)$, il existe $g_{y, i} \in G_{y}, r_{y, i} \in R_{x}\left(i=1, \cdots, k_{y}\right)$ tels que $f=\Sigma_{i} g_{y, i} \otimes r_{y, i}$ sur un voisinage de $y$. Comme $\pi$ est propre, on peut choisir $y_{1}, \cdots, y_{l} \in \pi^{-1}(x)$ pour représenter $f$. Soient $r_{i} \in R_{x}(i=1, \cdots, k)$ les éléments correspondant à $\left\{y_{j}\right\}_{j}$. Soit $R^{\prime}$ le sous faisceau de $R$ engendré par $r_{i}$ dans un voisinage $U^{\prime}$ de $x$, alors $R^{\prime}$ est un $\mathcal{O}_{Y}$-faisceau cohérent. Comme $f \in \pi_{*}\left(G \otimes \mathcal{O}_{z} \pi^{*} R^{\prime}\right)_{x}$, d'après i),

$$
f \in \tau_{x}\left(\pi_{*} G \otimes_{\mathcal{O}_{z}} R^{\prime}\right)_{x} \subset \tau_{x}\left(\pi_{*} G \otimes_{\mathcal{O}_{z}} R\right)_{x}
$$

On a bien démontré le Lemme 2.2 .

Les $\left(\mathcal{D}_{X}^{\bullet}(F), \bar{\partial}^{X}\right),\left(\mathcal{D}_{Z}^{\bullet}(F) \otimes_{\mathcal{O}_{Z}} \pi^{*} \mathcal{O}_{Y}^{\infty}, \bar{\partial}^{Z} \otimes 1\right),\left(\mathcal{D}_{Z}^{\bullet}(F) \otimes_{\mathcal{O}_{z}} \mathcal{D}_{X}^{\bullet}, \bar{\partial}^{Z} \otimes 1+1 \otimes \bar{\partial}^{X}\right)$ sont des résolutions acycliques du $\mathcal{O}_{Z}$-faisceau $F \otimes_{\mathcal{O}_{Z}} \pi^{*} \mathcal{O}_{Y}^{\infty}$, et donc pour $i \in\{0,1,2\}$, il existe un isomorphisme naturel [H, Proposition 3.1.2A],

$$
R^{\bullet} \pi_{*} F \otimes \mathcal{O}_{Y} \mathcal{O}_{Y}^{\infty} \simeq H\left(\mathcal{E}\left(\mathcal{F}_{i}\right)\right)
$$

Par (2.17), pour $i=(0,1,2)$, les $H\left(\mathcal{E}\left(\mathcal{F}_{i}\right)\right)$ sont canoniquement isomorphes.

Lemme 2.3. Pour $i=0,1, j_{i}: H\left(\mathcal{E}\left(\mathcal{F}_{i}\right)\right) \rightarrow H\left(\mathcal{E}\left(\mathcal{F}_{2}\right)\right)$ coïncide avec l'isomorphisme canonique $H\left(\mathcal{E}\left(\mathcal{F}_{i}\right)\right) \simeq H\left(\mathcal{E}\left(\mathcal{F}_{2}\right)\right)$.

PREUVE. Soit $\mathcal{E}_{r}\left(\mathcal{F}_{2}\right)$ (resp. $\mathcal{E}_{r}^{\prime}\left(\mathcal{F}_{2}\right)$ ) la suite spectrale associée au bicomplexe $\mathcal{E}\left(\mathcal{F}_{2}\right)$ filtré par

$$
F^{p} \mathcal{E}\left(\mathcal{F}_{2}\right)=\oplus_{p^{\prime} \geq p} \pi_{*}\left(\mathcal{D}_{Z}^{p^{\prime}} \otimes_{\mathcal{O}_{Z}} \mathcal{D}_{X}^{\bullet}\right) \quad\left(\operatorname{resp} . F^{\prime p} \mathcal{E}\left(\mathcal{F}_{2}\right)=\oplus_{p^{\prime} \geq p} \pi_{*}\left(\mathcal{D}_{Z}^{\bullet} \otimes_{\mathcal{O}_{Z}} \mathcal{D}_{X}^{p^{\prime}}\right)\right)
$$


Comme $\mathcal{D}_{Z}^{\bullet}(F)$ est une résolution du $\mathcal{O}_{Z}$-faisceau $F$, et comme les $\mathcal{D}_{Z}^{p}(p \geq 0)$ sont des $\mathcal{O}_{Z}$-faisceaux plats et fins, on sait que pour $p \geq 0, q>0$

$$
\mathcal{E}_{1}^{\prime p, 0}\left(\mathcal{F}_{2}\right)=\pi_{*}\left(F \otimes \mathcal{O}_{Z} \mathcal{D}_{X}^{p}\right), \quad \mathcal{E}_{1}^{\prime p, q}\left(\mathcal{F}_{2}\right)=0
$$

On a donc

$$
\mathcal{E}_{1}^{\prime p, 0}\left(\mathcal{F}_{2}\right)=\mathcal{E}^{p}\left(\mathcal{F}_{0}\right), \quad \mathcal{E}_{1}^{\prime p, q}\left(\mathcal{F}_{2}\right)=0 \quad \text { pour } \quad q>0
$$

Donc $j_{0}: \mathcal{E}\left(\mathcal{F}_{0}\right) \rightarrow \mathcal{E}\left(\mathcal{F}_{2}\right)$ induit un isomorphisme de $H\left(\mathcal{E}\left(\mathcal{F}_{0}\right)\right)$ sur $H\left(\mathcal{E}\left(\mathcal{F}_{2}\right)\right)$. De plus, sous l'identification (2.17), $j_{0}$ est l'identité.

D'après la preuve du lemme de Poincaré pour l'opérateur $\bar{\partial}$ [GrH, p25], on sait que $\mathcal{D}_{X}^{\bullet}$ est une résolution du $\mathcal{O}_{Z}$-faisceau $\pi^{*} \mathcal{O}_{Y}^{\infty}$. Comme les $\mathcal{D}_{Z}^{p}(F)(p \geq 0)$ sont des $\mathcal{O}_{Z}$-faisceaux plats et fins, on sait que pour $p \geq 0, q>0$

$$
\mathcal{E}_{1}^{p, 0}\left(\mathcal{F}_{2}\right)=\pi_{*}\left(\mathcal{D}_{Z}^{p}(F) \otimes_{\mathcal{O}_{Z}} \pi^{*} \mathcal{O}_{Y}^{\infty}\right), \quad \mathcal{E}_{1}^{p, q}\left(\mathcal{F}_{2}\right)=0
$$

Par le Lemme 2.2, on a

$$
\mathcal{E}_{1}^{p, 0}\left(\mathcal{F}_{2}\right)=\mathcal{E}_{1}^{p}\left(\mathcal{F}_{1}\right), \quad \mathcal{E}_{1}{ }^{p, q}\left(\mathcal{F}_{2}\right)=0 \quad \text { pour } \quad q>0
$$

Donc $j_{1}: \mathcal{E}\left(\mathcal{F}_{1}\right) \rightarrow \mathcal{E}\left(\mathcal{F}_{2}\right)$ induit un isomorphisme de $H\left(\mathcal{E}\left(\mathcal{F}_{1}\right)\right)$ sur $H\left(\mathcal{E}\left(\mathcal{F}_{2}\right)\right)$. De plus, sous l'identification (2.17), $j_{1}$ est l'identité.

On a bien démontré le Lemme 2.3.

Preuve du Théorème 2.1: Par (2.12) et par le Lemme 2.3, on sait que $j_{i}$ : $E_{1}\left(\mathcal{F}_{i}\right) \rightarrow E_{1}\left(\mathcal{F}_{2}\right)(i=0,1)$ est un isomorphisme canonique. Comme pour tout $r \geq 1$, les $j_{i}: E_{r}\left(\mathcal{F}_{i}\right) \rightarrow E_{r}\left(\mathcal{F}_{2}\right)$ sont des morphismes de complexes, on sait que pour tout $r \geq 1$, les $j_{i}$ sont bijectives. Donc $j_{1}^{-1} \circ j_{0}$ donne un isomorphisme $E_{r}\left(\mathcal{F}_{0}\right) \simeq E_{r}\left(\mathcal{F}_{1}\right)$. Donc la suite spectrale $\left(E_{r}^{\prime}, d_{r}^{\prime}\right)=\left(E_{r}\left(\mathcal{F}_{0}\right), d_{r}\right)(r \geq 2)$ est canoniquement isomorphe à la suite spectrale $\left(E_{r}, d_{r}\right)$.

Ceci termine la preuve du Théorème 2.1.

b) Le théorème principal. Soient $W, V, S$ des variétés complexes. Soient $\pi_{1}: W \rightarrow V, \pi_{2}: V \rightarrow S$ des submersions holomorphes de fibres compactes $X, Y$. Alors $\pi_{3}=\pi_{2} \circ \pi_{1}: W \rightarrow S$ est une submersion holomorphe de fibre compacte $Z$. Soit $\xi$ un fibré vectoriel holomorphe sur $W$. Soient $R^{\bullet} \pi_{1 *} \xi, R^{\bullet} \pi_{3 *} \xi, R^{\bullet} \pi_{2 *} R^{\bullet} \pi_{1 *} \xi$ les images directes de $\xi, \xi, R^{\bullet} \pi_{1 *} \xi$.

Pour $s \in S$, on note $\left(E_{r, s}, d_{r, s}\right)(r \geq 2)$ la suite spectrale de Leray associée à la fibration $Z_{s} \rightarrow Y_{s}$, $\xi$. Soit $n=\operatorname{dim} Z$.

On suppose que $R \pi_{1 *} \xi$ est localement libre $\operatorname{sur} V$, et que pour tout $r \geq 2, p, q \geq 0$, le rang de $E_{r}^{p, q}$ est localement constant sur $S$. Alors les $R \pi_{1 *} \xi, R \pi_{3 *} \xi, E_{r}(r \geq 2)$ sont des fibrés vectoriels holomorphes sur $V, S, S$.

Soit $\omega^{V}$ (resp. $\left.\omega^{W}\right)$ une $(1,1)$ forme réelle, fermée sur $V(\operatorname{resp} . W$ ), dont la restriction à chaque fibre $Y$ (resp. $Z$ ) définit une métrique hermitienne $g^{T Y}$ (resp. $g^{T Z}$ ) sur le fibré tangent relatif $T Y$ (resp. TZ). Comme dans [Ma1, §3(a)], on note $h^{R \pi_{1 *} \xi}, h^{H\left(Z, \xi_{\mid Z}\right)}, h^{E_{2}}$, les métriques $L^{2}$ sur $R \pi_{1 *} \xi, H\left(Z, \xi_{\mid Z}\right)=R \pi_{3 *} \xi, E_{2}$ associées à $g^{T Z}, g^{T Y}, h^{\xi}$.

Soient $T_{1}\left(\omega^{W}, h^{\xi}\right), T_{2}\left(\omega^{V}, h^{R \pi_{1 *} \xi}\right), T_{3}\left(\omega^{W}, h^{\xi}\right)$ les formes de torsion analytique construites dans Bismut-Köhler [BKö] sur $V, S, S$ associées à $\left(\pi_{1}, \omega^{W}, h^{\xi}\right)$, $\left(\pi_{2}, \omega^{V}, h^{R \pi_{1 *} \xi}\right),\left(\pi_{3}, \omega^{W}, h^{\xi}\right)$. 
Pour $s \in S, r \geq 2, E_{r+1, s}$ est la cohomologie de $\left(E_{r, s}, d_{r, s}\right)$. On définit la métrique $h^{E_{r}}$ sur $E_{r}$ comme à la Section 1. En effet, pour $r \geq 2$, soit $d_{r}^{*}$ l'adjoint de $d_{r}$ pour $h^{E_{r}}$. On pose

$$
D_{r}=d_{r}+d_{r}^{*}
$$

Alors, par la théorie de Hodge

$$
E_{r+1} \simeq \operatorname{Ker} D_{r} .
$$

Donc $E_{r+1}$ hérite de la métrique de $E_{r}$.

DÉFInition 2.4. On définit la classe de Bott-Chern $\widetilde{\operatorname{ch}}\left(E_{2}, H\left(Z, \xi_{\mid Z}\right), h^{E_{2}}\right.$, $\left.h^{H\left(Z, \xi_{\mid} Z\right)}\right) \in P^{S} / P^{S, 0}$ par la formule

$$
\begin{array}{r}
\tilde{\operatorname{ch}}\left(E_{2}, H\left(Z, \xi_{\mid Z}\right), h^{E_{2}}, h^{H\left(Z, \xi_{\mid Z}\right)}\right)=\Sigma_{r=2}^{\infty} \tilde{\operatorname{ch}}\left(E_{r}, E_{r+1}, h^{E_{r}}, h^{E_{r+1}}\right) \\
-\widetilde{\operatorname{ch}}\left(H\left(Z, \xi_{\mid Z}\right), E_{\infty}, h^{H\left(Z, \xi_{\mid}\right)}, h^{E_{\infty}}\right) .
\end{array}
$$

THÉORÈME 2.5. Si $\pi_{1}$ et $V$ sont projectives, les définitions de $\tilde{c h}\left(E_{2}, H\left(Z, \xi_{\mid Z}\right)\right.$, $\left.h^{E_{2}}, h^{H\left(Z, \xi_{\mid Z}\right)}\right)$ données dans [Ma1, Définition 10.15] et dans (2.24) sont équivalentes.

PREUVE. Soit $(\xi)_{0 \leq i \leq n}$ une résolution $\pi_{1 *}$ et $\pi_{3 *}$ acyclique de fibrés holomorphes de $\xi$ sur $W$, et $\mathcal{F}=\left(\mathcal{F}^{i, j}\right)_{0 \leq i, j \leq n}$ une résolution $\pi_{2 *}$ acyclique de fibrés holomorphes du complexe $\left(R^{0} \pi_{1 *} \xi^{i}\right)$ au sens de [CE, chap. XVII]. Si on filtre le bicomplexe $E(\mathcal{F})=$ $R^{0} \pi_{2 *} \mathcal{F}$ par $F^{p} E(\mathcal{F})=\oplus_{p^{\prime} \geq p} R^{0} \pi_{2 *} \mathcal{F}^{\bullet}, p^{\prime}$, alors la suite spectrale associée $\left(E_{r}(\mathcal{F}), d_{r}\right)$ calcule la suite spectrale de Leray $\left(E_{r}, d_{r}\right)$ (à partir de $r=2$ ) [Ma1, $\left.\S 10(\mathrm{c})\right]$. On se donne des métriques hermitiennes $h^{E^{p, q}(\mathcal{F})}, h^{E_{1}^{p, q}(\mathcal{F})}$ sur $E^{p, q}(\mathcal{F})=R^{0} \pi_{2 *} \mathcal{F}^{p, q}$, $E_{1}^{p, q}(\mathcal{F})$.

Dans [Ma1, Définition 10.15], on a défini $\widetilde{\operatorname{ch}}\left(E_{2}, H\left(Z, \xi_{\mid Z}\right), h^{E_{2}}, h^{H\left(Z, \xi_{\mid} Z\right.}\right) \in$ $P^{S} / P^{S, 0}$ par

$$
\widetilde{\operatorname{ch}}\left(E(\mathcal{F}), H(E(\mathcal{F})), h^{E(\mathcal{F})}, h^{H\left(Z, \xi_{\mid} Z\right)}\right)-\sum_{i=0}^{1} \widetilde{\operatorname{ch}}\left(E_{i}(\mathcal{F}), E_{i+1}(\mathcal{F}), h^{E_{i}(\mathcal{F})}, h^{E_{i+1}(\mathcal{F})}\right)
$$

Par Théorème 1.2, on sait que le terme à droite de (2.24) est égal à (2.25). Ceci démontre notre résultat.

Pour la commodité du lecteur, nous énonçons de nouveau le Théorème 0.1.

THÉORÈME 2.6. On a l'identité suivante:

$$
\begin{aligned}
& T_{3}\left(\omega^{W}, h^{\xi}\right)-T_{2}\left(\omega^{V}, h^{R \pi_{1 *} \xi}\right)-\int_{Y} \operatorname{Td}\left(T Y, g^{T Y}\right) T_{1}\left(\omega^{W}, h^{\xi}\right) \\
& \quad+\int_{Z} \widehat{T d}\left(T Z, T Y, g^{T Z}, g^{T Y}\right) \operatorname{ch}\left(\xi, h^{\xi}\right) \\
& \quad-\operatorname{ch}\left(E_{2}, H\left(Z, \xi_{\mid Z}\right), h^{E_{2}}, h^{H\left(Z, \xi_{\mid} Z\right)}\right)=0 \text { dans } \quad P^{S} / P^{S, 0} .
\end{aligned}
$$

Preuve. Le reste de cette Section est consacré à la preuve du Théorème 2.6.

REMARQUe 2.7. Si $\pi_{1}$ et $V$ sont projectives, par le Théorème 2.5 , les résultats de $[\mathrm{Ma1}, \S 12]$ impliquent le Théorème 2.6. Donc, si $\pi_{1}$ et $V$ sont projectives, et si les $E_{r}(r \geq 2)$ sont des fibrés vectoriels holomorphes sur $S$, on obtiendra ainsi deux preuves distinctes du Théorème 2.6. On peut aussi considérer que si les hypothèses de [Ma1] et celles du Théorème 2.6 sont vérifiées simultanément, on obtient une autre démonstration du Théorème 2.5 . 
Par le Théorème 2.1, on peut noter sans inconvénient $\left(E_{r, s}, d_{r, s}\right)(r \geq 0, s \in S)$ la suite spectrale associée au complexe filtré $\left(\Omega\left(Z_{s}, \xi_{\mid Z_{s}}\right), \bar{\partial}^{Z}\right)$. Alors par [Ma1, (5.5)], $E_{0}=\Omega\left(Y, \Omega\left(X, \xi_{\mid X}\right)_{\mid Y}\right)$. On munit $E_{0}$ de la métrique $h^{E_{0}}$ construite en [Ma1, (5.4)]. On rappelle que ${ }^{0} \nabla^{\Omega\left(Z, \xi_{\mid Z}\right)}$ est la connexion sur $\Omega\left(Z, \xi_{\mid Z}\right)$ définie en [Ma1, (5.13)]. On désigne aussi $\nabla^{E_{0}}$ la connexion sur $E_{0}$ correspondant à [Ma1, (5.5)].

Dans la suite, on identifie $E_{r}$ à un sous-fibré vectoriel de $E_{0}$ comme à la Section $1(\mathrm{a}),[\mathrm{BerB}, \S 6]$. Alors on a une suite de sous-fibrés hermitiens de $E_{0}, E_{0} \supset E_{1} \cdots \supset$ $E_{r} \supset \cdots$.

Pour $r \geq 1$, soit $E_{r}^{\perp}$ l'espace orthogonal à $E_{r}$ dans $E_{r-1}$. Alors $D_{r}(r \geq 2)$ agit sur $E_{r+1}^{\perp}$ comme un opérateur inversible. Pour $r \geq 1$, soit $p_{r}$ la projection orthogonale de $E_{0}$ sur $E_{r}$. On pose $p_{r}^{\perp}=1-p_{r}$. Soit $\nabla^{E_{r}}(r \geq 2)$ la connexion holomorphe hermitienne sur $\left(E_{r}, h^{E_{r}}\right)$. Alors, en utilisant [B2, Proposition 1.8], [Ma1, (5.25)], comme en (1.10), on a

$$
\nabla^{E_{r}}=p_{r} \nabla^{E_{0}} p_{r}
$$

Pour $u>0, r>1$, on pose

$$
\mathcal{D}_{r, u}=\nabla^{E_{r}}+u D_{r}
$$

c) Des résultats intermédiaires. On utilise les mêmes notations que dans [Ma1, §4]. Comme dans [Ma1, §4], on supposera que $S$ est compacte.

ThÉoRÈme 2.8. i) Pour tout $u>0$,

$$
\lim _{T \rightarrow+\infty} \varphi \operatorname{Tr}_{s}\left[N_{3, u, T} \exp \left(-B_{3, u, T}^{2}\right)\right]=\varphi \operatorname{Tr}_{s}\left[\left(N_{2, u}+N_{X}\right) \exp \left(-B_{2, u}^{2}\right)\right] .
$$

ii) Pour tout $u>0$, il existe $C, \delta>0$ tels que pour tout $T \geq 1$,

$$
\begin{array}{r}
\left|\varphi \operatorname{Tr}_{s}\left[M_{3, u, T} \exp \left(-B_{3, u, T}^{2}\right)\right]-\frac{2}{T} \varphi \operatorname{Tr}_{s}\left[\left(N_{X}-\operatorname{dim} X\right) \exp \left(-B_{2, u}^{2}\right)\right]\right| \\
\leq \frac{C}{T^{\delta+1}} .
\end{array}
$$

iii) Pour tout $0<u_{1}<u_{2}$ fixés, il existe $C>0$ tel que pour $u \in\left[u_{1}, u_{2}\right], T \geq 1$,

$$
\left|\varphi \operatorname{Tr}_{s}\left[N_{3, u, T} \exp \left(-B_{3, u, T}^{2}\right)\right]\right| \leq C .
$$

PreUVE. L'inégalité (2.30) a déjà été montrée à [Ma1, §5(i)]. En utilisant [Ma1, (5.43), (5.45), (5.63) et (5.67)], on a aussi les inégalités (2.29) et (2.31).

On a terminé la preuve du Théorème 2.8 .

Pour $r>1$, on pose

$$
\operatorname{ch}^{\prime}\left(E_{r}, h^{E_{r}}\right)=\varphi \operatorname{Tr}_{s}\left[N_{Z} \exp \left(-\nabla^{E_{r}, 2}\right)\right]
$$

On rappelle que $h_{T}^{H\left(Z, \xi_{\mid Z}\right)}$ est la métrique $L^{2}$ sur $H\left(Z, \xi_{\mid Z}\right)$ associée à $g_{T}^{T Z}, h^{\xi}$, et que $\nabla_{T}^{H\left(Z, \xi_{\mid Z}\right)}$ est la connexion holomorphe hermitienne sur $\left(H\left(Z, \xi_{\mid Z}\right), h_{T}^{H\left(Z, \xi_{\mid Z}\right)}\right)$. On rappelle aussi que $\zeta\left(E_{r}, h^{E_{r}}\right) \in P^{S}$ a été défini dans [Ma1, Définition 10.3].

On a l'analogue des Théorèmes 1.6 et 1.7. 


\section{THÉORÈME 2.9. On a l'identité suivante}

$$
\begin{gathered}
\lim _{T \rightarrow+\infty}\left\{\int_{1}^{+\infty} 2\left\{\varphi \operatorname{Tr}_{s}\left[N_{3, u^{2}, T} \exp \left(-B_{3, u^{2}, T}^{2}\right)\right]-\varphi \operatorname{Tr}_{s}\left[N_{Z} \exp \left(-\nabla_{T}^{H\left(Z, \xi_{\mid Z}\right), 2}\right)\right]\right\} \frac{d u}{u}\right. \\
\left.\quad-2 \Sigma_{r \geq 2}(r-1)\left[\operatorname{ch}^{\prime}\left(E_{r}, h^{E_{r}}\right)-\operatorname{ch}^{\prime}\left(E_{r+1}, h^{E_{r+1}}\right)\right] \log T\right\} \\
=\int_{1}^{+\infty}\left\{\varphi \operatorname{Tr}_{s}\left[\left(N_{2, u}+N_{X}\right) \exp \left(-B_{2, u}^{2}\right)\right]-\operatorname{ch}^{\prime}\left(E_{2}, h^{E_{2}}\right)\right\} \frac{d u}{u} \\
-\Sigma_{r \geq 2} \zeta\left(E_{r}, h^{E_{r}}\right)+\Gamma^{\prime}(1)\left[\operatorname{ch}^{\prime}\left(E_{2}, h^{E_{2}}\right)-\operatorname{ch}^{\prime}\left(E_{\infty}, h^{E_{\infty}}\right)\right] .
\end{gathered}
$$

THÉORÈmE 2.10. Il existe $\delta>0$ tel que, quand $T \rightarrow+\infty$, on a

$$
\begin{aligned}
\varphi \operatorname{Tr}_{s} & {\left[Q_{T}^{H\left(Z, \xi_{\mid Z}\right)} \exp \left(-\nabla_{T}^{H\left(Z, \xi_{\mid Z}\right), 2}\right)\right] } \\
& =-\varphi \operatorname{Tr}_{s}\left[\frac{2\left(N_{X}-\operatorname{dim} X\right)}{T} \exp \left(-\nabla^{E_{\infty}, 2}\right)\right]+O\left(\frac{1}{T^{1+\delta}}\right) .
\end{aligned}
$$

De plus

$$
\begin{array}{r}
\int_{1}^{+\infty}\left\{\varphi \operatorname{Tr}_{s}\left[Q_{T}^{H\left(Z, \xi_{\mid Z}\right)} \exp \left(-\nabla_{T}^{H\left(Z, \xi_{\mid Z}\right), 2}\right)\right]+\varphi \operatorname{Tr}_{s}\left[\frac{2\left(N_{X}-\operatorname{dim} X\right)}{T} \exp \left(-\nabla^{E_{\infty}, 2}\right)\right]\right\} d T \\
=\tilde{\operatorname{ch}}\left(H\left(Z, \xi_{\mid Z}\right), E_{\infty}, h^{H\left(Z, \xi_{\mid Z}\right)}, h^{E_{\infty}}\right) \text { dans } P^{S} / P^{S, 0} .
\end{array}
$$

PREUVE. Les preuves des Théorèmes 2.9 et 2.10 sont différées aux Sections 2(f) et $2(\mathrm{~g})$.

d) Preuve du Théorème 2.6. On procède comme dans [Ma1, §4]. Les termes $I_{3}^{0}$ et $I_{4}^{0}$ ont déjà été traités dans [Ma1, $\left.\S 4\right]$. D'après les Théorèmes $2.8-2.10$, on peut aussi traiter les termes $I_{1}^{0}$ et $I_{2}^{0}$ comme on l'a fait dans la Section $1(\mathrm{~d})$.

Comme dans la Section $1(\mathrm{~d})$, en combinant la preuve du Théorème 2.9, et [B4, $\S 6.6-6.8]$, on peut traiter aussi le terme à droite de [Ma1,(4.8)]. Donc il existe $\theta_{1}^{3}, \theta_{2}^{3}$, $\theta_{3}^{3}$ des formes universelles explicites sur $S$ telles qu'on a

$$
\sum_{i=1}^{4} I_{i}^{3}=\bar{\partial} \theta_{1}^{3}-\partial \theta_{2}^{3}-\bar{\partial} \partial \theta_{3}^{3} .
$$

En procédant comme dans [Ma1, §4], on obtient le Théorème 2.6.

e) L'asymptotique de certaines supertraces quand $T \rightarrow+\infty$ ou $u \rightarrow 0$. On utilise les mêmes notations que dans [Ma1, §5].

On rappelle que $p_{T}$ est la projection de $E_{0}$ sur $\operatorname{Ker} D_{T}^{X}$ définie dans [Ma1, Définition 5.6], $p_{T}^{\perp}=1-p_{T}$, et que la norme ||$_{0}$ sur $E_{0}^{0}$ est définie dans [Ma1, $\S 5(\mathrm{f})]$. Soit \|\|$^{0,0}$ la norme sur $L\left(E_{0}^{0}, E_{0}^{0}\right)$ associée à | $\left.\right|_{0}$. On rappelle que pour $T>0$, l'opérateur $A_{T}^{(0)}=T D_{T}^{X}+D_{T}^{H}$ est défini en [Ma1, (5.16)].

Pour $r \geq 2, s \in S$, soit $\operatorname{Sp} D_{r, s}^{2}$ (resp. $\operatorname{Sp} D_{r, s}^{2,>0}$ ) le spectre (resp. le spectre non nul) de $D_{r, s}^{2}$. Dans la suite, on fixe $c_{1}, c_{2}>0$ tels que

$$
\left.\underset{r \in S}{\cup_{r \geq 2}} \operatorname{Sp} D_{r, s}^{2,>0} \subset\right] c_{1}, c_{2}[\text { et }] 0,2 c_{1}\left[\cap \cup_{s \in S} \operatorname{Sp} D_{s}^{Y, 2}=\phi .\right.
$$

Soient $\delta, \Delta \subset \mathbf{C}$ les contours définis comme on l'a fait après (1.45). Soit $\delta^{\prime}, \Delta^{\prime}$ les domaines de bords par $\delta, \Delta$. Soit $U_{1} \subset \mathbf{C}$ défini comme en (1.28).

Le résultat suivant est établi dans [BerB, Théorème 6.5]. 
Proposition 2.11. Pour $r \geq 2, \lambda \in U_{1}, s \in E_{0}^{0}$, quand $T \rightarrow+\infty$, on a

$$
\left(\lambda-T^{r-1} A_{T}^{(0)}\right)^{-1} s \rightarrow p_{r}\left(\lambda-D_{r}\right)^{-1} p_{r} s .
$$

Par (2.37), pour $r \geq 2$, les valeurs propres de $A_{T}^{(0)}$ qui sont $O\left(\frac{1}{T^{r-1}}\right)$ sont de la forme $\frac{1}{T^{r-1}}\left(\mathrm{Sp} D_{r}+O\left(\frac{1}{T}\right)\right)$. D'où, pour $T \gg 1$

$$
\operatorname{Sp} A_{T}^{(0), 2} \cap\left[0,2 c_{1}\left[\subset \frac{\delta^{\prime}}{T^{2(n-1)}} \cup \cup_{i=2}^{n} \frac{\Delta^{\prime}}{T^{2(i-1)}} .\right.\right.
$$

Pour $r \geq 2, T \gg 1$, on pose

$$
\begin{aligned}
& \widetilde{p}_{r, T}=\frac{1}{2 \pi i} \int_{\left\{\lambda \in \mathbf{C},|\lambda|=\sqrt{c_{2}}\right\}}\left(\lambda-T^{r-1} A_{T}^{(0)}\right)^{-1} d \lambda, \\
& \widetilde{p}_{r, T}^{\perp}=1-\widetilde{p}_{r, T}, \\
& q_{r, T}=\widetilde{p}_{r, T}-\widetilde{p}_{r+1, T} .
\end{aligned}
$$

Pour $r \geq 2$, soit $\widetilde{E}_{r, T}$ la somme directe des espaces propres de $A_{T}^{(0), 2}$ associés aux valeurs propres $\lambda \in\left[0, c_{2} / T^{2(r-1)}\right]$. Alors $\widetilde{p}_{r, T}$ est la projection orthogonale de $E_{0}$ sur $\widetilde{E}_{r, T}$. Pour $T \gg 1, \widetilde{E}_{r, T}$ est un fibré vectoriel sur $S$ et $\operatorname{dim} \widetilde{E}_{r, T}=\operatorname{dim} E_{r}$. Pour $r \geq 3$, soit $\widetilde{E}_{r, T}^{\perp}$ l'espace orthogonal à $\widetilde{E}_{r, T}$ dans $\widetilde{E}_{r-1, T}$. Soit $\widetilde{E}_{2, T}^{\perp}$ l'espace orthogonal à $\widetilde{E}_{2, T}$ dans $E_{0}$.

Pour $T \gg 1, r \geq 2$, soit $Q_{r, T}^{\prime}: E_{r} \rightarrow \widetilde{E}_{r, T}$ l'application linéaire

$$
s \in E_{r} \rightarrow Q_{r, T}^{\prime} s=\widetilde{p}_{r, T} s \in \widetilde{E}_{r, T} .
$$

Évidemment, l'application $Q_{r, T}^{\prime}(r \geq 2)$ est $\mathcal{C}^{\infty}$ sur $S$. D'après (2.37), pour $T$ assez grand, $Q_{r, T}^{\prime}$ est injective pour tout $s \in S$. Comme $\operatorname{dim} \widetilde{E}_{r, T}=\operatorname{dim} E_{r}, Q_{r, T}^{\prime}$ est un isomorphisme de $E_{r}$ sur $\widetilde{E}_{r, T}$. Soit $Q_{r, T}^{\prime *}$ l'adjoint de $Q_{r, T}^{\prime}$. On pose

$$
\begin{aligned}
& R_{r, T}=\left(Q_{r, T}^{\prime *} Q_{r, T}^{\prime}\right)^{1 / 2} \\
& J_{r, T}=Q_{r, T}^{\prime} R_{r, T}^{-1} .
\end{aligned}
$$

Alors $J_{r, T}$ est une isométrie linéaire de $E_{r}$ sur $\widetilde{E}_{r, T}$, et $J_{r, T}^{-1}=R_{r, T}^{-1} Q_{r, T}^{\prime *}$.

Soit $\widetilde{p}_{r, T}\left(x, x^{\prime}\right), p_{r}\left(x, x^{\prime}\right)\left(x, x^{\prime} \in Z_{s}, s \in S\right)$ les noyaux $\mathcal{C}^{\infty}$ des opérateurs $\widetilde{p}_{r, T}, p_{r}$ relativement à $d v_{Z}\left(x^{\prime}\right) /(2 \pi)^{\operatorname{dim} Z}$.

Proposition 2.12. Pour tout $k \in \mathbf{N}$, il existe $\delta>0$ tel que sous la norme $\mathcal{C}^{k}$, pour $r \geq 2, T \gg 1$, on a

$$
\widetilde{p}_{r, T}\left(x, x^{\prime}\right)=p_{r}\left(x, x^{\prime}\right)+O\left(\frac{1}{T^{\delta}}\right) .
$$

Preuve. i) Par (2.38), (2.39), pour $T \gg 1$, on a

$$
\begin{aligned}
\tilde{p}_{2, T} & =\frac{1}{2 \pi i} \int_{\left\{\lambda \in \mathbf{C},|\lambda|=c_{1}\right\}}\left(\lambda-A_{T}^{(0), 2}\right)^{-1} d \lambda \\
& =\frac{1}{2 \pi i} \int_{\left\{\lambda \in \mathbf{C},|\lambda|=\sqrt{c_{1}}\right\}}\left(\lambda-A_{T}^{(0)}\right)^{-1} d \lambda .
\end{aligned}
$$

En procédant comme dans [Ma1, $\S 5(\mathrm{f})$ et $\S 5(\mathrm{~g})]$, on sait que $\widetilde{p}_{2, T}\left(x, x^{\prime}\right)\left(x, x^{\prime} \in Z_{s}\right)$ et ses dérivées sont uniformément bornées pour $s \in S, x, x^{\prime} \in Z_{s}, T \gg 1$. 
Par [BerB, (5.75), (5.85), (5.86), (5.90), (5.91)], on voit que pour $\lambda \in \mathbf{C},|\lambda|=$ $\sqrt{c_{1}}$, on a

$$
\left\|\left(\lambda-A_{T}^{(0)}\right)^{-1}-p_{1}\left(\lambda-D^{Y}\right)^{-1} p_{1}\right\|^{0,0} \leq \frac{C}{T} .
$$

De (2.42) et (2.43), on tire que

$$
\left\|\widetilde{p}_{2, T}-p_{2}\right\|^{0,0} \leq \frac{C}{T}
$$

En appliquant la méthode de [BL, §11(p)], pour $r=2$, on obtient (2.41).

ii) Pour $r \geq 2$, par (2.38),

(2.45) $\tilde{p}_{r, T}=J_{2, T} p_{2}\left[\frac{1}{2 \pi i} \int_{\left\{\lambda \in \mathbf{C},|\lambda|=\sqrt{c_{2}}\right\}} J_{2, T}^{-1}\left(\lambda-T^{r-1} A_{T}^{(0)}\right)^{-1} J_{2, T} d \lambda\right] J_{2, T}^{-1} \widetilde{p}_{2, T}$.

Soit $\left(J_{2, T} p_{2}\right)\left(x, x^{\prime}\right),\left(J_{2, T}^{-1} \widetilde{p}_{2, T}\right)\left(x, x^{\prime}\right)\left(x, x^{\prime} \in Z_{s}, s \in S\right)$ les noyaux $\mathcal{C}^{\infty}$ des opérateurs $J_{2, T} p_{2}, J_{2, T}^{-1} \widetilde{p}_{2, T}$ relativement à $d v_{Z}\left(x^{\prime}\right) /(2 \pi)^{\operatorname{dim} Z}$. En utilisant (2.41) pour $r=2$, on a

$$
\begin{aligned}
& \left(J_{2, T} p_{2}\right)\left(x, x^{\prime}\right)=p_{2}\left(x, x^{\prime}\right)+O\left(\frac{1}{T^{\delta}}\right), \\
& \left(J_{2, T}^{-1} \widetilde{p}_{2, T}\right)\left(x, x^{\prime}\right)=p_{2}\left(x, x^{\prime}\right)+O\left(\frac{1}{T^{\delta}}\right) .
\end{aligned}
$$

Soit $\left(E_{k, T, s}, d_{k, T, s}\right)(s \in S, k \geq 0)$ la suite spectrale associée à $\Omega\left(Z_{s}, \xi_{\mid Z_{s}}\right)$ construite dans [BerB, $\S 6(\mathrm{a})]$. Soit $p_{k, T}(s), D_{k, T}(s), \psi_{k, \lambda, T}(s)=\left(\psi_{k, \lambda, T}^{i}(s)\right)_{0 \leq i \leq k}(k \geq 2, s \in$ $S$ ) les opérateurs définis dans [BerB, $\S 6(\mathrm{a}),(\mathrm{b})]$. On note $p_{0, T}=\mathrm{Id}_{E_{0}}, p_{1, T}=p_{T}$. Soit $B_{T}$ l'opérateur défini dans [BerB, §5(a)]. Par la construction de [BerB, §6(a)], $Q_{k, T}=p_{k, T} B_{T}:\left(E_{k}, d_{k}\right) \rightarrow\left(E_{k, T}, d_{k, T}\right)(k \geq 0)$ est un isomorphisme de complexes. Soit $d_{k}^{*}, Q_{k, T}^{*}$ les adjoints de $d_{k}, Q_{k, T}$. Alors

$$
\begin{aligned}
& Q_{k, T}^{-1}=\left(Q_{k, T}^{*} Q_{k, T}\right)^{-1} Q_{k, T}^{*}, \\
& D_{k, T}=Q_{k, T} d_{k} Q_{k, T}^{-1}+\left(Q_{k, T}^{-1}\right)^{*} d_{k}^{*} Q_{k, T}^{*} .
\end{aligned}
$$

Soit $p_{k, T}\left(x, x^{\prime}\right)\left(x, x^{\prime} \in Z_{s}, s \in S, k \geq 2\right)$ le noyau $\mathcal{C}^{\infty}$ de $p_{k, T}$ relativement à $d v_{Z}\left(x^{\prime}\right) /(2 \pi)^{\operatorname{dim} Z}$. En utilisant [BerB, (5.47), (6.6)], [Ma1, (5.27)], pour $k \geq 2$, on a

$$
p_{k, T}\left(x, x^{\prime}\right)=p_{k}\left(x, x^{\prime}\right)+O\left(\frac{1}{T^{2}}\right) .
$$

D'après [BerB, Théorème 6.2], pour $\mu \in \mathbf{R}, \psi_{k, \lambda, T}$ est une application linéaire de $E_{0}^{\mu}$ dans $\left(E_{0}^{\mu}\right)^{k+1}$ dont la norme est uniformément bornée pour $T \geq 1$. Par [BerB, (6.49)], on a

$$
\begin{aligned}
\left(\lambda-T^{r-1} A_{T}^{(0)}\right)^{-1} & =\left(\lambda-D_{r, T}\right)^{-1} p_{r, T}+\Sigma_{i=1}^{r} \psi_{r, \lambda, T}^{i} / T^{i} \\
& +\left(\lambda-T^{r-1} A_{T}^{(0)}\right)^{-1}\left(-\lambda \Sigma_{i=1}^{r} \psi_{r, \lambda, T}^{i} / T^{i}+D_{T}^{H} \psi_{r, \lambda, T}^{r} / T\right) .
\end{aligned}
$$

Dans la suite, on va montrer que

$$
\frac{1}{2 \pi i} \int_{\left\{\lambda \in \mathbf{C},|\lambda|=\sqrt{c_{2}}\right\}} J_{2, T}^{-1}\left(\lambda-T^{r-1} A_{T}^{(0)}\right)^{-1} J_{2, T} d \lambda=p_{r \mid E_{2}}+O\left(\frac{1}{T^{\delta}}\right) .
$$

- D'après (2.41) pour $r=2,(2.48),(2.49)$, on a (2.50) pour la norme $\mathcal{C}^{0}$. 
- Soit $U$ une section $\mathcal{C}^{\infty}$ de $T_{\mathbf{R}} S$. On rappelle que la connexion $\nabla^{E_{0}}$ sur $E_{0}$ est définie à la Section $2(\mathrm{~b})$. On note $\nabla^{\operatorname{End}\left(E_{0}\right)}$ la connexion sur $\operatorname{End}\left(E_{0}\right)$ associée à $\nabla^{E_{0}}$. Par (2.49), on a

$$
\begin{gathered}
\nabla_{U}^{\operatorname{End}\left(E_{0}\right)}\left(\lambda-T^{r-1} A_{T}^{(0)}\right)^{-1}=\nabla_{U}^{\operatorname{End}\left(E_{0}\right)}\left(\lambda-D_{r, T}\right)^{-1} p_{r, T}+\Sigma_{i=1}^{r} \nabla_{U}^{\operatorname{End}\left(E_{0}\right)} \psi_{r, \lambda, T}^{i} / T^{i} \\
(2.51) \quad+\left(\lambda-T^{r-1} A_{T}^{(0)}\right)^{-1}\left(-\lambda \Sigma_{i=1}^{r} \nabla_{U}^{\operatorname{End}\left(E_{0}\right)} \psi_{r, \lambda, T}^{i} / T^{i}+\nabla_{U}^{\operatorname{End}\left(E_{0}\right)} D_{T}^{H} \psi_{r, \lambda, T}^{r} / T\right) \\
+\left(\nabla_{U}^{\operatorname{End}\left(E_{0}\right)}\left(\lambda-T^{r-1} A_{T}^{(0)}\right)^{-1}\right)\left(-\lambda \Sigma_{i=1}^{r} \psi_{r, \lambda, T}^{i} / T^{i}+D_{T}^{H} \psi_{r, \lambda, T}^{r} / T\right) .
\end{gathered}
$$

On remplace $\nabla_{U}^{\operatorname{End}\left(E_{0}\right)}\left(\lambda-T^{r-1} A_{T}^{(0)}\right)^{-1}$ dans le dernier terme à droite en utilisant de nouveau la formule ci-dessus, et on répète l'opération $r$ fois. Alors il existe $\theta_{i}, f_{i}, g_{i}$ $\left(1 \leq i \leq(r+1)^{2}\right)$ qui , pour tout $\mu \in \mathbf{R}$, sont des applications linéaires de $E_{0}^{\mu}$ dans $E_{0}^{\mu-(r+\overline{2})}$ dont les normes sont uniformément bornées pour $T \geq 1$, telles que

$$
\begin{aligned}
& \nabla_{U}^{\operatorname{End}\left(E_{0}\right)}\left(\lambda-T^{r-1} A_{T}^{(0)}\right)^{-1}=\nabla_{U}^{\operatorname{End}\left(E_{0}\right)}\left(\lambda-D_{r, T}\right)^{-1} p_{r, T} \\
& \quad+\Sigma_{i \geq 1} \theta_{i} / T^{i}+\left(\lambda-T^{r-1} A_{T}^{(0)}\right)^{-1} \Sigma_{i \geq 1} f_{i} / T^{i} \\
& \quad+\frac{1}{T^{r}}\left(\lambda-T^{r-1} A_{T}^{(0)}\right)^{-1}\left[\nabla_{U}^{E_{0}}, T^{r-1} A_{T}^{(0)}\right]\left(\lambda-T^{r-1} A_{T}^{(0)}\right)^{-1} \Sigma_{i \geq 1} g_{i} / T^{i}
\end{aligned}
$$

L'opérateur $\left[\nabla_{U}^{E_{0}}, A_{T}^{(0)}\right]$ est un opérateur différentiel d'ordre 1 le long de la fibre $Z$.

Par [Ma1, (5.56)], il existe $C, C_{0}, C_{1}>0$, tels que pour $\lambda \in U_{1}, s \in E_{0}$, on a

$$
\begin{aligned}
& \left|\left(\lambda-T^{r-1} A_{T}^{(0)}\right) s\right|_{0} \geq T^{r-1}\left(C_{0}|| s \|_{E_{0}^{1}}-C_{1}|s|_{0}\right) \\
& \left|\left(\lambda-T^{r-1} A_{T}^{(0)}\right)^{-1} s\right|_{0} \leq C|s|_{0} .
\end{aligned}
$$

Donc

$$
\left\|\left(\lambda-T^{r-1} A_{T}^{(0)}\right)^{-1} s\right\|_{E_{0}^{1}} \leq C|s|_{0} .
$$

De i), (2.47), (2.52), (2.53), (2.54), on tire (2.50) pour la norme $\mathcal{C}^{1}$. 1).

- On répète les arguments ci-dessus, alors on obtient (2.50) pour la norme $\mathcal{C}^{k}(k \geq$

De i), (2.45), (2.46) et (2.50), on tire (2.41) pour $r \geq 2$.

On a montré la Proposition 2.12.

On rappelle que $\nabla_{T}^{\Omega\left(Z, \xi_{\mid Z}\right)}$ est la connexion sur $\Omega\left(Z, \xi_{\mid Z}\right)$ définie en [Ma1, (5.12)], et que les opérateurs $A_{u, T}$ et $A_{T}^{(0)}$ sont définis en [Ma1, (5.14) et (5.16)]. On pose

$$
A_{r, u, T}=A_{T^{r-1} u, T}=C_{T} B_{3,\left(T^{r-1} u\right)^{2}, T} C_{T}^{-1} .
$$

Soit $A_{r, 1, T}^{(0)}$ (resp. $A_{r, 1, T}^{(>0)}$ ) la partie de $A_{r, 1, T}$ de degré 0 (resp. $>0$ ) dans $\Lambda\left(T_{\mathbf{R}}^{*} S\right)$. Par [Ma1, (5.14) et (5.16)], on a

$$
\begin{aligned}
& A_{r, 1, T}^{(0)}=T^{r-1} A_{T}^{(0)} \\
& A_{r, 1, T}^{(>0)}=C_{T}\left(\nabla_{T}^{\Omega(Z, \xi \mid Z)}-\frac{1}{2 \sqrt{2} T^{r-1}} c_{T}\left(T_{3, T}\right)\right) C_{T}^{-1}
\end{aligned}
$$

On pose

$$
R_{r, 1, T}=\left[A_{r, 1, T}^{(0)}, A_{r, 1, T}^{(>0)}\right]+A_{r, 1, T}^{(>0), 2}
$$


Par [Ma1, (5.21), (5.23)], quand $T \rightarrow+\infty$, on a

$$
\begin{aligned}
& C_{T} \nabla_{T}^{\Omega\left(Z, \xi_{\mid Z}\right)} C_{T}^{-1}={ }^{0} \nabla^{\Lambda\left(T^{*(0,1)} Z\right) \otimes \xi}+O\left(\frac{1}{T}\right) \\
& C_{T} c_{T}\left(T_{3, T}\right) C_{T}^{-1}=c\left(T_{2}\right)+O\left(\frac{1}{T}\right) .
\end{aligned}
$$

On rappelle que le produit hermitien \langle\rangle$_{0}$ sur $E_{0}^{0}$ (resp. la norme ||$_{T, 1}$ sur $E_{0}^{1}$ ) est défini dans $[\mathrm{Ma} 1, \S 5(\mathrm{f})]$, et que ${ }^{0} \nabla^{\Lambda\left(T^{*(0,1)} Z\right) \otimes \xi}$ est la connexion sur $\Lambda\left(T^{*(0,1)} Z\right) \otimes \xi$ définie en [Ma1, §5(b)].

DÉFInITION 2.13. Pour $2 \leq r \leq n, T \gg 1, s \in E_{0}$, on pose

$$
|s|_{r, T, 1}^{2}=\left|\widetilde{p}_{r, T} s\right|_{0}^{2}+\sum_{k=2}^{r-1} T^{2(r-k)}\left|q_{k, T} s\right|_{0}^{2}+T^{2(r-1)}\left|\widetilde{p}_{2, T}^{\perp} s\right|_{T, 1}^{2} .
$$

Comme dans [Ma1, §5(f)], soit ||$_{r, T,-1}$ la norme sur $E_{0}^{-1}$ associée à ||$_{r, T, 1}$.

On a l'analogue de [Ma1, Théorèmes 5.19-5.25].

THÉORÈme 2.14. Il existe $C_{1}, C_{2}, C_{3}>0, T_{0}>0$ tels que pour $T \geq T_{0}, s, s^{\prime} \in$ $\Lambda\left(T_{\mathbf{R}}^{*} S\right) \widehat{\otimes} E_{0}$,

$$
\begin{gathered}
\left|T^{r-1} A_{T}^{(0)} s\right|_{0}^{2} \geq C_{1}|s|_{r, T, 1}^{2}-C_{2}|s|_{0}^{2} \\
T^{2(r-1)}\left|\left\langle A_{T}^{(0)} s, A_{T}^{(0)} s^{\prime}\right\rangle_{0}\right| \leq C_{3}|s|_{r, T, 1}\left|s^{\prime}\right|_{r, T, 1} \\
\left|\left\langle R_{r, 1, T} s, s^{\prime}\right\rangle_{0}\right| \leq C_{3}\left(|s|_{r, T, 1}\left|s^{\prime}\right|_{0}+|s|_{0}\left|s^{\prime}\right|_{r, T, 1}\right)
\end{gathered}
$$

PreUVE. Soit $\left(T^{k-1} A_{T}^{(0)} \widetilde{p}_{k, T}\right)\left(x, x^{\prime}\right),\left(D_{k} p_{k}\right)\left(x, x^{\prime}\right)\left(x, x^{\prime} \in Z_{s}, s \in S, k \geq 2\right)$ les noyaux $\mathcal{C}^{\infty}$ des opérateurs $T^{k-1} A_{T}^{(0)} \widetilde{p}_{k, T}, D_{k} p_{k}$ relativement à $d v_{Z}\left(x^{\prime}\right) /(2 \pi)^{\operatorname{dim} Z}$. Par (2.38), pour $T \gg 1, k \geq 2$, on a

$$
T^{k-1} A_{T}^{(0)} \widetilde{p}_{k, T}=J_{2, T} p_{2}\left[\frac{1}{2 \pi i} \int_{\left\{\lambda \in \mathbf{C},|\lambda|=\sqrt{c_{2}}\right\}} \lambda J_{2, T}^{-1}\left(\lambda-T^{k-1} A_{T}^{(0)}\right)^{-1} J_{2, T} d \lambda\right] J_{2, T}^{-1} \widetilde{p}_{2, T}
$$

En utilisant (2.46), en procédant comme dans la preuve de (2.50), on a

$$
\left(T^{k-1} A_{T}^{(0)} \widetilde{p}_{k, T}\right)\left(x, x^{\prime}\right)=\left(D_{k} p_{k}\right)\left(x, x^{\prime}\right)+O\left(\frac{1}{T^{\delta}}\right) .
$$

Donc

$$
T^{k-1} A_{T}^{(0)} q_{k, T}=D_{k} p_{k+1}^{\perp} p_{k}+O\left(\frac{1}{T^{\delta}}\right) .
$$

Pour $s, s^{\prime} \in \Lambda\left(T_{\mathbf{R}}^{*} S\right) \widehat{\otimes} E_{0}$, on a

$$
\begin{aligned}
T^{2(r-1)}\left\langle A_{T}^{(0)} s, A_{T}^{(0)} s^{\prime}\right\rangle_{0} & =T^{2(r-1)}\left\langle A_{T}^{(0)} \widetilde{p}_{2, T}^{\perp} s, A_{T}^{(0)} \widetilde{p}_{2, T}^{\perp} s^{\prime}\right\rangle_{0} \\
& +T^{2(r-1)} \Sigma_{k=2}^{r-1}\left\langle A_{T}^{(0)} q_{k, T} s, A_{T}^{(0)} q_{k, T} s^{\prime}\right\rangle_{0} \\
& +T^{2(r-1)}\left\langle A_{T}^{(0)} \widetilde{p}_{r, T} s, A_{T}^{(0)} \widetilde{p}_{r, T} s^{\prime}\right\rangle_{0} .
\end{aligned}
$$

En utilisant [Ma1, (5.56)], (2.62)-(2.64), pour $T \gg 1$, on a

$$
\begin{aligned}
\left|T^{r-1} A_{T}^{(0)} s\right|_{0}^{2} \geq T^{2(r-1)}\left(C_{1}^{\prime}\left|q_{2, T}^{\perp} s\right|_{T, 1}^{2}-C\left|q_{2, T}^{\perp} s\right|_{0}^{2}\right) \\
+\Sigma_{k=2}^{r-1} C_{1}^{\prime} T^{2(r-k)}\left|q_{k, T} s\right|_{0}^{2}+\left|T^{r-1} A_{T}^{(0)} \widetilde{p}_{r, T} s\right|_{0}^{2}
\end{aligned}
$$


Par (2.38), (2.39) et (2.64), pour $T \gg 1$, on a

$$
\left|T^{r-1} A_{T}^{(0)} s\right|_{0}^{2} \geq T^{2(r-1)}\left|A_{T}^{(0)} q_{2, T}^{\perp} s\right|_{0}^{2} \geq C_{0} T^{2(r-1)}\left|q_{2, T}^{\perp} s\right|_{0}^{2}
$$

De (2.65), (2.66), on tire la première équation de (2.60).

En utilisant [Ma1, (5.56)], (2.62)-(2.64), pour $T \gg 1$,

$$
\begin{aligned}
T^{2(r-1)}\left|\left\langle A_{T}^{(0)} s, A_{T}^{(0)} s^{\prime}\right\rangle_{0}\right| \leq & C_{3} T^{2(r-1)}\left|\widetilde{p}_{2, T}^{\perp} s\right|_{T, 1}\left|\widetilde{p}_{2, T}^{\perp} s^{\prime}\right|_{T, 1} \\
& +\Sigma_{k=2}^{r-1} C_{3} T^{2(r-k)}\left|q_{k, T} s\right|_{0}\left|q_{k, T} s^{\prime}\right|_{0} \\
& +\left|T^{r-1} A_{T}^{(0)} \widetilde{p}_{r, T} s\right|_{0}\left|T^{r-1} A_{T}^{(0)} \widetilde{p}_{r, T} s^{\prime}\right|_{0} \\
\leq & C_{3}|s|_{r, T, 1}\left|s^{\prime}\right|_{r, T, 1}
\end{aligned}
$$

En utilisant (2.41), (2.56), (2.58), (2.62) et (2.63), pour $k, k^{\prime} \geq 2, T \gg 1$, comme en [Ma1, (5.56)], on a

$$
\begin{aligned}
& \left|\left\langle\left[A_{r, 1, T}^{(0)}, A_{r, 1, T}^{(>0)}\right] \widetilde{p}_{2, T}^{\perp} s, \widetilde{p}_{2, T}^{\perp} s^{\prime}\right\rangle_{0}\right| \leq C T^{r-1}\left(\left|\widetilde{p}_{2, T}^{\perp} s\right|_{T, 1}\left|\widetilde{p}_{2, T}^{\perp} s^{\prime}\right|_{0}\right. \\
& \left.+\left|\widetilde{p}_{2, T}^{\perp} s\right|_{0}\left|\tilde{p}_{2, T}^{\perp} s^{\prime}\right|_{T, 1}\right), \\
& \left.\left\langle\left[A_{r, 1, T}^{(0)}, A_{r, 1, T}^{(>0)}\right] \widetilde{p}_{2, T} s, \widetilde{p}_{2, T}^{\perp} s^{\prime}\right\rangle_{0}\left|\leq C T^{r-1}\right| \widetilde{p}_{2, T} s\right|_{0}\left|\widetilde{p}_{2, T}^{\perp} s^{\prime}\right|_{T, 1}, \\
& \left|\left\langle\left[A_{r, 1, T}^{(0)}, A_{r, 1, T}^{(>0)}\right] q_{k, T} s, q_{k^{\prime}, T} s^{\prime}\right\rangle_{0}\right| \leq C\left(T^{r-k}+T^{r-k^{\prime}}\right)\left|q_{k, T} s\right|_{0}\left|q_{k^{\prime}, T} s^{\prime}\right|_{0} \text {. }
\end{aligned}
$$

On a une inégalité analogue à la dernière équation de (2.68), si on remplace $q_{k, T}$ ou $q_{k^{\prime}, T}$ par $\widetilde{p}_{r, T}$.

En utilisant $(2.56),(2.58)$ et $(2.68)$, pour $T \gg 1$, on a

$$
\begin{aligned}
\left|\left\langle R_{r, 1, T} s, s^{\prime}\right\rangle_{0}\right| & \leq C|| s||_{E_{0}^{1}}\left|s^{\prime}\right|_{0}+\left|\left\langle\left[A_{r, 1, T}^{(0)}, A_{r, 1, T}^{(>0)}\right] s, s^{\prime}\right\rangle_{0}\right| \\
\leq & C_{3}\left(|s|_{r, T, 1}\left|s^{\prime}\right|_{0}+|s|_{0}\left|s^{\prime}\right|_{r, T, 1}\right) .
\end{aligned}
$$

On a bien terminé la preuve du Théorème 2.14.

Soit $U_{1}, \cdots, U_{m}$ (resp. $U_{1}^{\prime}, \cdots, U_{m^{\prime}}^{\prime}$ ) une famille de sections $\mathcal{C}^{\infty}$ de $T_{\mathbf{R}} Y$ (resp. $\left.T_{\mathbf{R}} X\right)$ telle que pour tout $y \in V$ (resp. $x \in W$ ) $U_{1}(y), \cdots, U_{m}(y)$ (resp. $U_{1}^{\prime}(x), \cdots$, $\left.U_{m^{\prime}}^{\prime}(x)\right)$ engendrent $\left(T_{\mathbf{R}} Y\right)_{y}$ (resp. $\left.\left(T_{\mathbf{R}} X\right)_{x}\right)$. On rappelle que $\mathcal{D}_{T}$ est la famille d'opérateurs agissant sur $E_{0}$ définie en [Ma1, (5.60)],

$$
\mathcal{D}_{T}=\left\{p_{T}^{0} \nabla_{U_{l, 1}^{H}}^{\Lambda\left(T^{*(0,1)} Z\right) \otimes \xi} p_{T}, p_{T}^{\perp 0} \nabla_{U_{l, 1}^{H}}^{\Lambda\left(T^{*(0,1)} Z\right) \otimes \xi} p_{T}^{\perp}, p_{T}^{\perp 0} \nabla_{U_{i}^{\prime}}^{\Lambda\left(T^{*(0,1)} Z\right) \otimes \xi} p_{T}^{\perp}\right\} .
$$

DÉFINITION 2.15. Pour $T>0$, soit $\mathcal{D}_{T}^{\prime}$ la famille d'opérateurs agissant sur $E_{0}$

$$
\mathcal{D}_{T}^{\prime}=\left\{\tilde{p}_{2, T}^{\perp} Q \tilde{p}_{2, T}^{\perp}, Q \in \mathcal{D}_{T}\right\} .
$$

Théonème 2.16. Pour tout $k \in \mathbf{N}$ fixé, il existe $C_{k}>0$ tel que pour $T \geq$ $1, Q_{1}, \cdots, Q_{k} \in \mathcal{D}_{T}^{\prime}, s, s^{\prime} \in \Lambda\left(T_{\mathbf{R}}^{*} S\right) \widehat{\otimes} E_{0}$, on $a$

$$
\left|\left\langle\left[Q_{1},\left[Q_{2}, \cdots\left[Q_{k}, A_{r, 1, T}^{2}\right], \cdots\right]\right] s, s^{\prime}\right\rangle_{0}\right| \leq C_{k}|s|_{r, T, 1}\left|s^{\prime}\right|_{r, T, 1}
$$

Preuve. On considère d'abord le cas où $k=1$. Soit $Q \in \mathcal{D}_{T}$. 
Les opérateurs $\left[{ }^{0} \nabla_{U_{l, 1}^{H}}^{\Lambda\left(T^{*(0,1)} Z\right) \otimes \xi}, p_{T}\right],{ }^{0} \nabla_{U_{i}^{\prime}}^{\Lambda\left(T^{*(0,1)} Z\right) \otimes \xi} p_{T}, p_{T}{ }^{0} \nabla_{U_{i}^{\prime}}^{\Lambda\left(T^{*(0,1)} Z\right) \otimes \xi}$ (resp. $\left.Q \widetilde{p}_{2, T}, \widetilde{p}_{2, T} Q\left(Q \in \mathcal{D}_{T}\right)\right)$ sont des opérateurs dont les restrictions aux fibres $X_{b}(b \in V)$ (resp. $Z_{s},(s \in S)$ ) ont des noyaux $\mathcal{C}^{\infty}$ uniformément bornés pour $\left(x, x^{\prime}\right) \in X_{b} \times X_{b}, b \in$ $V, T \geq 1$ (resp. $\left.\left(x, x^{\prime}\right) \in Z_{s} \times Z_{s}, s \in S, T \gg 1\right)$.

1) - $\operatorname{Par}(2.39)$ et $(2.56)$, on a

$$
\left[\widetilde{p}_{2, T}^{\perp} Q \widetilde{p}_{2, T}^{\perp}, A_{r, 1, T}^{(0), 2}\right]=\widetilde{p}_{2, T}^{\perp}\left[Q, A_{r, 1, T}^{(0), 2}\right] \widetilde{p}_{2, T}^{\perp}
$$

D'après [Ma1, Théorème 5.21], (2.56) et (2.72), pour $s, s^{\prime} \in \Lambda\left(T_{\mathbf{R}}^{*} S\right) \widehat{\otimes} E_{0}$, on a

$$
\left|\left\langle\left[\widetilde{p}_{2, T}^{\perp} Q \widetilde{p}_{2, T}^{\perp}, A_{r, 1, T}^{(0), 2}\right] s, s^{\prime}\right\rangle_{0}\right| \leq C T^{2(r-1)}\left|\widetilde{p}_{2, T}^{\perp} s\right|_{T, 1}\left|\widetilde{p}_{2, T}^{\perp} s^{\prime}\right|_{T, 1} \cdot
$$

- Soit

$$
\begin{aligned}
G_{T} & =\left[C_{T} \nabla_{T}^{\Omega\left(Z, \xi_{\mid Z}\right)} C_{T}^{-1}, A_{T}^{(0)}\right] \\
H_{T} & =\left[C_{T} \frac{1}{2 \sqrt{2} T^{r-1}} c_{T}\left(T_{3, T}\right) C_{T}^{-1}, A_{r, 1, T}^{(0)}\right]
\end{aligned}
$$

Alors $G_{T}, H_{T}$ sont des opérateurs différentiels d'ordre 1 le long de la fibre $Z$, et $G_{T}$ est la partie de $A_{T}^{2}$ de degré 1 dans $\Lambda\left(T_{\mathbf{R}}^{*} S\right)$.

Par [Ma1, (5.16), (5.36)], (2.56) et (2.58), les coefficients de $H_{T}$ sont uniformément bornés pour $T \geq 1$. Par la Proposition 2.12, pour $s, s^{\prime} \in \Lambda\left(T_{\mathbf{R}}^{*} S\right) \widehat{\otimes} E_{0}$, on a

$$
\begin{aligned}
&\left|\left\langle\left[\widetilde{p}_{2, T}^{\perp} Q \widetilde{p}_{2, T}^{\perp}, H_{T}\right] s, s^{\prime}\right\rangle_{0}\right|=\left|\left\langle\left(\left[Q, H_{T}\right]-\left[Q \widetilde{p}_{2, T}+\widetilde{p}_{2, T} Q \widetilde{p}_{2, T}^{\perp}, H_{T}\right]\right) s, s^{\prime}\right\rangle_{0}\right| \\
& \leq C|| s \|_{E_{0}^{1}}\left|s^{\prime}\right|_{0} .
\end{aligned}
$$

- Par (2.56) et (2.58), $A_{r, 1, T}^{(>0), 2}$ est un opérateur différentiel d'ordre 1 le long de la fibre $Z$, dont les coefficients sont uniformément bornés pour $T \geq 1$. D'où, pour $s, s^{\prime} \in \Lambda\left(T_{\mathbf{R}}^{*} S\right) \widehat{\otimes} E_{0}$, on a

$$
\left|\left\langle\left[\widetilde{p}_{2, T}^{\perp} Q \widetilde{p}_{2, T}^{\perp}, A_{r, 1, T}^{(>0), 2}\right] s, s^{\prime}\right\rangle_{0}\right| \leq C\|s\|_{E_{0}^{1}}\left|s^{\prime}\right|_{0} .
$$

2) Pour montrer le Théorème 2.16 dans le cas où $k=1$, par (2.56), (2.72)-(2.76), il suffit de montrer que pour $Q \in \mathcal{D}_{T}, s, s^{\prime} \in \Lambda\left(T_{\mathrm{R}}^{*} S\right) \widehat{\otimes} E_{0}$, on a

$$
\left|\left\langle\left[\widetilde{p}_{2, T}^{\perp} Q \widetilde{p}_{2, T}^{\perp}, T^{r-1} G_{T}\right] s, s^{\prime}\right\rangle_{0}\right| \leq C|s|_{r, T, 1}\left|s^{\prime}\right|_{r, T, 1}
$$

i) Pour $s, s^{\prime} \in \Lambda\left(T_{\mathbf{R}}^{*} S\right) \widehat{\otimes} E_{0}$, on a

$$
\begin{aligned}
\left\langle\left[\widetilde{p}_{2, T}^{\perp} Q \widetilde{p}_{2, T}^{\perp}, G_{T}\right] s, s^{\prime}\right\rangle_{0}=\left\langle\left[\widetilde{p}_{2, T}^{\perp} Q \widetilde{p}_{2, T}^{\perp}, G_{T}\right] \widetilde{p}_{2, T}^{\perp} s, \widetilde{p}_{2, T}^{\perp} s^{\prime}\right\rangle_{0} \\
+\left\langle\left[\widetilde{p}_{2, T}^{\perp} Q \widetilde{p}_{2, T}^{\perp}, G_{T}\right] \widetilde{p}_{2, T} s, \widetilde{p}_{2, T}^{\perp} s^{\prime}\right\rangle_{0} \\
+\left\langle\left[\widetilde{p}_{2, T}^{\perp} Q \widetilde{p}_{2, T}^{\perp}, G_{T}\right] \widetilde{p}_{2, T}^{\perp} s, \widetilde{p}_{2, T} s^{\prime}\right\rangle_{0}
\end{aligned}
$$

ii) $\operatorname{Par}(2.39)$, on a

$$
Q \widetilde{p}_{2, T}^{\perp} G_{T}-G_{T} \widetilde{p}_{2, T}^{\perp} Q=\left[Q, G_{T}\right]-Q \widetilde{p}_{2, T} G_{T}+G_{T} \tilde{p}_{2, T} Q .
$$


Par [Ma1, Théorème 5.21], (2.41), (2.58) et (2.79), on a

$$
\begin{aligned}
\left|\left\langle\left[\widetilde{p}_{2, T}^{\perp} Q \widetilde{p}_{2, T}^{\perp}, G_{T}\right] \widetilde{p}_{2, T}^{\perp} s, \widetilde{p}_{2, T}^{\perp} s^{\prime}\right\rangle_{0}\right| \\
\quad=\left|\left\langle\left(Q \widetilde{p}_{2, T}^{\perp} G_{T}-G_{T} \widetilde{p}_{2, T}^{\perp} Q\right) \widetilde{p}_{2, T}^{\perp} s, \widetilde{p}_{2, T}^{\perp} s^{\prime}\right\rangle_{0}\right|^{\perp} \\
\leq C\left(\left|\widetilde{p}_{2, T}^{\perp} s\right|_{T, 1}\left|\widetilde{p}_{2, T}^{\perp} s^{\prime}\right|_{T, 1}+T\left|\widetilde{p}_{2, T}^{\perp} s\right|_{0}\left|\widetilde{p}_{2, T}^{\perp} s^{\prime}\right|_{0}\right) .
\end{aligned}
$$

iii) $\bullet$ Si $Q=p_{T}^{\perp 0} \nabla_{U_{l, 1}^{H}}^{\Lambda\left(T^{*(0,1)} Z\right) \otimes \xi} p_{T}^{\perp}$ ou $p_{T}^{\perp 0} \nabla_{U_{i}^{\prime}}^{\Lambda\left(T^{*(0,1)} Z\right) \otimes \xi} p_{T}^{\perp}$, alors, par (2.41), on a

$$
\begin{gathered}
\left|\left\langle\left[\widetilde{p}_{2, T}^{\perp} Q \widetilde{p}_{2, T}^{\perp}, G_{T}\right] \widetilde{p}_{2, T} s, \widetilde{p}_{2, T}^{\perp} s^{\prime}\right\rangle_{0}\right|=\left|\left\langle Q \widetilde{p}_{2, T}^{\perp} G_{T} \widetilde{p}_{2, T} s, p_{T}^{\perp} \widetilde{p}_{2, T}^{\perp} s^{\prime}\right\rangle_{0}\right| \\
\leq T\left|\widetilde{p}_{2, T} s\right|_{0}\left|p_{T}^{\perp} \widetilde{p}_{2, T}^{\perp} s^{\prime}\right|_{0} .
\end{gathered}
$$

- Soit $Q=p_{T}^{0} \nabla_{U_{l, 1}^{H}}^{\Lambda\left(T^{*(0,1)} Z\right) \otimes \xi} p_{T}$. Par (2.74), on a

$(2.82) p_{T} G_{T} \widetilde{p}_{2, T}=\left[p_{T} D_{T}^{H} C_{T} \nabla_{T}^{\Omega\left(Z, \xi_{\mid Z}\right)} C_{T}^{-1}-C_{T} \nabla_{T}^{\Omega\left(Z, \xi_{\mid Z}\right)} C_{T}^{-1}\left(A_{T}^{(0)} \widetilde{p}_{2, T}\right)\right] \widetilde{p}_{2, T}$.

D'après (2.58), (2.62) et (2.82), le noyau de $p_{T} G_{T} \widetilde{p}_{2, T}$ est $\mathcal{C}^{\infty}$, uniformément borné pour $T \gg 1$. De même, par (2.62), le noyau de $\widetilde{p}_{2, T} G_{T} \widetilde{p}_{2, T}$ est $\mathcal{C}^{\infty}$, uniformément borné pour $T \gg 1$. Donc

$$
\begin{aligned}
& \left|\left\langle\left[\widetilde{p}_{2, T}^{\perp} Q \widetilde{p}_{2, T}^{\perp}, G_{T}\right] \widetilde{p}_{2, T} s, \widetilde{p}_{2, T}^{\perp} s^{\prime}\right\rangle_{0}\right|=\left|\left\langle Q \widetilde{p}_{2, T}^{\perp} G_{T} \widetilde{p}_{2, T} s, p_{T} \widetilde{p}_{2, T}^{\perp} s^{\prime}\right\rangle_{0}\right| \\
& =\left|\left\langle Q p_{T} G_{T} \widetilde{p}_{2, T} s-Q \widetilde{p}_{2, T} G_{T} \widetilde{p}_{2, T} s, p_{T} \widetilde{p}_{2, T}^{\perp} s^{\prime}\right\rangle_{0}\right| \\
& \leq C\left|\widetilde{p}_{2, T} s\right|_{0}\left|p_{T} \widetilde{p}_{2, T}^{\perp} s^{\prime}\right|_{0} .
\end{aligned}
$$

iv) Comme en iii), on a aussi

$$
\left|\left\langle\left[\widetilde{p}_{2, T}^{\perp} Q \widetilde{p}_{2, T}^{\perp}, G_{T}\right] \widetilde{p}_{2, T}^{\perp} s, \widetilde{p}_{2, T} s^{\prime}\right\rangle_{0}\right| \leq C\left|\widetilde{p}_{2, T}^{\perp} s\right|_{T, 1}\left|\widetilde{p}_{2, T} s^{\prime}\right|_{0} .
$$

De $(2.60), \mathrm{i})$-iv), on tire (2.77). On a montré le Théorème 2.16 pour $k=1$.

3) Les commutateurs d'ordre supérieur. général.

On répète les arguments ci-dessus, et on obtient le Théorème 2.16 dans le cas

On a bien terminé la preuve du Théorème 2.16.

Pour $r \geq 2, T \gg 1$, on pose

$$
\bar{F}_{r, u, T}=\frac{1}{2 \pi i} \int_{\Delta} e^{-u^{2} \lambda}\left(\lambda-A_{r, 1, T}^{2}\right)^{-1} d \lambda .
$$

Soit $\bar{F}_{r, u, T}\left(x, x^{\prime}\right)\left(x, x^{\prime} \in Z_{s}\right)$ le noyau $\mathcal{C}^{\infty}$ de l'opérateur $\bar{F}_{r, u, T}$ relativement à $d v_{Z}\left(x^{\prime}\right) /(2 \pi)^{\operatorname{dim} Z}$.

THÉORÈme 2.17. Pour tout $k \in \mathrm{N}, u_{0}>0$, il existe $C>0, C^{\prime}>0$ tels que pour $u \geq u_{0}, T \geq T_{0}, x, x^{\prime} \in Z_{s}$,

$$
\sup _{\substack{|\alpha| \leq k \\\left|\alpha^{\prime}\right| \leq k}}\left|\frac{\partial^{|\alpha|+\left|\alpha^{\prime}\right|}}{\partial x^{\alpha} \partial{x^{\prime}}^{\alpha^{\prime}}} \bar{F}_{r, u, T}\left(x, x^{\prime}\right)\right| \leq C \exp \left(-C^{\prime} u^{2}\right) .
$$

PREUVE. En utilisant les arguments qu'on a donnés au début de la preuve du Théorème 2.16, en utilisant les Théorèmes 2.14 et 2.16, et en procédant comme dans la preuve de [B4, Théorème 9.20$]$, [BL, Théorème 13.32], on obtient le Théorème. 
Soit $E_{r, T}^{\perp}$ l'espace orthogonal à $\widetilde{E}_{r, T}$ dans $E_{0}$. Si $A$ est un opérateur linéaire de $E_{0}$ dans $E_{0}$, on écrit $A$ sous forme matricielle relativement au scindage $E_{0}=\widetilde{E}_{r, T} \oplus E_{r, T}^{\perp}$

$$
A=\left[\begin{array}{ll}
A_{1} & A_{2} \\
A_{3} & A_{4}
\end{array}\right]
$$

tel que $A_{1}=\widetilde{p}_{r, T} A \widetilde{p}_{r, T}, A_{2}=\widetilde{p}_{r, T} A \widetilde{p}_{r, T}^{\perp}, \cdots$.

Par (2.27), (2.41), (2.58) et (2.62), quand $T \rightarrow+\infty$, on a

$$
\begin{aligned}
& A_{r, 1, T, 1}^{2}=p_{r} D_{r}^{2} p_{r}+p_{r}\left[D_{r}, \nabla^{E_{r}}\right] p_{r}+p_{r}{ }^{0} \nabla^{\Omega\left(Z, \xi_{\mid Z}\right), 2} p_{r}+O\left(\frac{1}{T^{\delta}}\right) \\
& A_{r, 1, T, 2}^{2}=\widetilde{p}_{r, T}\left[C_{T}\left(\nabla_{T}^{\Omega\left(Z, \xi_{\mid Z}\right)}-\frac{1}{2 \sqrt{2} T^{r-1}} c_{T}\left(T_{3, T}\right)\right) C_{T}^{-1}, T^{r-1} A_{T}^{(0)}\right] \tilde{p}_{r, T}^{\perp}+O(1) \\
& A_{r, 1, T, 3}^{2}=\widetilde{p}_{r, T}^{\perp}\left[C_{T}\left(\nabla_{T}^{\Omega\left(Z, \xi_{\mid Z}\right)}-\frac{1}{2 \sqrt{2} T^{r-1}} c_{T}\left(T_{3, T}\right)\right) C_{T}^{-1}, T^{r-1} A_{T}^{(0)}\right] \tilde{p}_{r, T}+O(1) \\
& A_{r, 1, T, 4}^{2}=T^{2(r-1)} A_{T, 4}^{(0), 2}+R_{r, 1, T, 4}
\end{aligned}
$$

On note aussi ||$_{r, T, 0}$ la norme ||$_{0}$ sur $E_{0}^{0}$. Pour $A \in L\left(E_{0}^{k}, E_{0}^{k^{\prime}}\right)\left(k, k^{\prime} \in\right.$ $\{-1,0,1\})$, on note $\|A\|_{r, T}^{k, k^{\prime}}$ la norme de $A$ associée à ||$_{r, T, k}$ et ||$_{r, T, k^{\prime}}$.

ThÉORÈme 2.18. Il existe $\delta, C>0, T_{0}>0$ tels que pour tout $\lambda \in U_{1}, T \geq$ $T_{0}, r \geq 2$,

$$
\left\|A_{r, 1, T, 1}^{2}+A_{r, 1, T, 2}^{2}\left(\lambda^{2}-A_{r, 1, T, 4}^{2}\right)^{-1} A_{r, 1, T, 3}^{2}-\tilde{p}_{r, T} J_{r, T} \mathcal{D}_{r, 1}^{2} J_{r, T}^{-1} \tilde{p}_{r, T}\right\|_{r, T}^{1,-1} \leq \frac{C}{T^{\delta}}
$$

PREUVE. Par (2.39), (2.60), et en procédant comme en [B4, Théorème 9.22], pour $r \geq 2, \lambda \in \mathbf{C},|\lambda| \leq 2 \sqrt{c_{2}}$, on a

$$
\begin{aligned}
& \left\|\left(\lambda^{2}-T^{r-1} A_{T, 4}^{(0)}\right)^{-1}\right\|_{r, T}^{0,0} \leq \frac{C}{T}, \\
& \left\|\left(\lambda^{2}-T^{2(r-1)} A_{T, 4}^{(0), 2}\right)^{-1}\right\|_{r . T}^{-1,0} \leq \frac{C}{T}, \\
& \left\|\left(\lambda^{2}-T^{2(r-1)} A_{T, 4}^{(0), 2}\right)^{-1}\right\|_{r . T}^{0.1} \leq \frac{C}{T}, \\
& \left\|\left(\lambda^{2}-T^{2(r-1)} A_{T, 4}^{(0), 2}\right)^{-1}\right\|_{r . T}^{-1.1} \leq C .
\end{aligned}
$$

De $(2.59),(2.60)$, on tire que

$$
\begin{aligned}
& \left\|A_{r, 1, T, 2}^{2}\right\|_{r, T}^{1,-1} \leq C, \\
& \left\|A_{r, 1, T, 3}^{2}\right\|_{r, T}^{1,-1} \leq C, \\
& \left\|R_{r, 1, T, 4}\right\|_{r, T}^{1,-1} \leq \frac{C}{T} .
\end{aligned}
$$

On a aussi

$$
\begin{aligned}
& \left(\lambda-A_{r, 1, T, 4}^{2}\right)^{-1}=\left(\lambda-T^{2(r-1)} A_{T, 4}^{(0), 2}\right)^{-1} \\
& \quad+\left(\lambda-T^{2(r-1)} A_{T, 4}^{(0), 2}\right)^{-1} \Sigma_{i=1}^{2 \operatorname{dim} S}\left(R_{r, 1, T, 4}\left(\lambda-T^{2(r-1)} A_{T, 4}^{(0), 2}\right)^{-1}\right)^{i}
\end{aligned}
$$


De (2.89), (2.90) et (2.91), on tire que pour $r \geq 2, \lambda \in U_{1}, T \gg 1$, on a

$$
\begin{aligned}
& \left\|\left(\lambda^{2}-A_{r, 1, T, 4}^{2}\right)^{-1}\right\|_{r, T}^{-1,1} \leq C, \\
& \left\|\left(\lambda^{2}-A_{r, 1, T, 4}^{2}\right)^{-1}-\left(\lambda^{2}-T^{2(r-1)} A_{T, 4}^{(0), 2}\right)^{-1}\right\|_{r, T}^{-1,1} \leq \frac{C}{T}, \\
& \left\|\left(\lambda^{2}-T^{2(r-1)} A_{T, 4}^{(0), 2}\right)^{-1}+\left(T^{2(r-1)} A_{T, 4}^{(0), 2}\right)^{-1}\right\|_{r, T}^{-1,1} \leq \frac{C}{T} .
\end{aligned}
$$

Pour simplifier, on note la connexion $C_{T} \nabla_{T}^{\Omega\left(Z, \xi_{\mid Z}\right)} C_{T}^{-1}$ sur $\Omega\left(Z, \xi_{\mid Z}\right)$ par $\nabla_{T}$. En utilisant (2.58), (2.59) et (2.68), on a aussi

$$
\begin{aligned}
& \left\|\left[C_{T} \frac{1}{2 \sqrt{2} T^{r-1}} c_{T}\left(T_{3, T}\right) C_{T}^{-1}, T^{r-1} A_{T}^{(0)}\right]\right\|_{r, T}^{1,-1} \leq \frac{C}{T^{r-1}}, \\
& \left\|\left[\nabla_{T}, T^{r-1} A_{T}^{(0)}\right]\right\|_{r, T}^{1,-1} \leq C .
\end{aligned}
$$

D'après (2.27), (2.28), (2.87), (2.90), (2.92) et (2.93), pour montrer (2.88), il suffit de montrer que

$$
\begin{gathered}
\| \widetilde{p}_{r, T}\left[\nabla_{T}, T^{r-1} A_{T}^{(0)}\right] \tilde{p}_{r, T}^{\perp}\left(T^{2(r-1)} A_{T, 4}^{(0), 2}\right)^{-1} \widetilde{p}_{r, T}^{\perp}\left[\nabla_{T}, T^{r-1} A_{T}^{(0)}\right] \tilde{p}_{r, T} \\
-\widetilde{p}_{r, T} J_{r, T} p_{r} \nabla^{E_{0}} p_{r}^{\perp} \nabla^{E_{0}} p_{r} J_{r, T}^{-1} \widetilde{p}_{r, T} \|_{r, T}^{1,-1} \leq \frac{C}{T^{\delta}} .
\end{gathered}
$$

On a

$$
\widetilde{p}_{r, T}\left[\nabla_{T}, T^{r-1} A_{T}^{(0)}\right] \widetilde{p}_{r, T}^{\perp}\left(T^{2(r-1)} A_{T, 4}^{(0), 2}\right)^{-1} \widetilde{p}_{r, T}^{\perp}\left[\nabla_{T}, T^{r-1} A_{T}^{(0)}\right] \widetilde{p}_{r, T}
$$

$=\widetilde{p}_{r, T} \nabla_{T} \widetilde{p}_{r, T}^{\perp} \nabla_{T} \widetilde{p}_{r, T}$

$+\widetilde{p}_{r, T} T^{r-1} A_{T}^{(0)} \tilde{p}_{r, T}\left[\nabla_{T}, \tilde{p}_{r, T}\right] \widetilde{p}_{r, T}^{\perp}\left(T^{2(r-1)} A_{T, 4}^{(0), 2}\right)^{-1} \widetilde{p}_{r, T}^{\perp}\left[\nabla_{T}, T^{r-1} A_{T}^{(0)}\right] \widetilde{p}_{r, T}$

$+\widetilde{p}_{r, T}\left[\nabla_{T}, \widetilde{p}_{r, T}\right] \widetilde{p}_{r, T}^{\perp}\left(T^{r-1} A_{T, 4}^{(0)}\right)^{-1} \widetilde{p}_{r, T}^{\perp}\left[\nabla_{T}, \widetilde{p}_{r, T}\right] \widetilde{p}_{r, T} T^{r-1} A_{T}^{(0)} \widetilde{p}_{r, T}$.

Par (2.41), (2.58), $\left[\nabla_{T}, \widetilde{p}_{r, T}\right]$ est un opérateur différentiel d'ordre 0 uniformément borné pour $T \gg 1$.

De (2.41),(2.58), (2.59), (2.62), (2.93) et (2.95), on tire (2.94).

On a bien terminé la preuve du Théorème 2.18 .

On rappelle que $\psi_{u}$ est l'application de $\Lambda\left(T_{\mathbf{R}}^{*} S\right)$ sur $\Lambda\left(T_{\mathbf{R}}^{*} S\right)$ définie avant (1.47). Pour $2 \leq r \leq n=\operatorname{dim} Z, T \geq T_{0}$, on pose

$$
\begin{aligned}
F_{r, u, T} & =\frac{1}{2 \pi i} \psi_{u} \varphi \operatorname{Tr}_{s}\left[N_{3, T^{2(r-1)}, T} \int_{\Delta} e^{-u^{2} \lambda}\left(\lambda-A_{r, 1, T}^{2}\right)^{-1} d \lambda\right] \\
F_{r, u, \infty} & =\frac{1}{2 \pi i} \psi_{u} \varphi \operatorname{Tr}_{s}\left[N_{Z} \int_{\Delta} e^{-u^{2} \lambda}\left(\lambda-\mathcal{D}_{r, 1}^{2}\right)^{-1} d \lambda\right] \\
G_{r, u, T} & =\frac{1}{2 \pi i} \psi_{u} \varphi \operatorname{Tr}_{s}\left[N_{3, T^{2(r-1)}, T} \int_{\delta} e^{-u^{2} \lambda}\left(\lambda-A_{r, 1, T}^{2}\right)^{-1} d \lambda\right] \quad \text { pour } r \geq 1, \\
G_{1, u, \infty} & =\frac{1}{2 \pi i} \psi_{u} \varphi \operatorname{Tr}_{s}\left[\left(N_{2,1}+N_{X}\right) \int_{\delta} e^{-u^{2} \lambda}\left(\lambda-B_{2,1}^{2}\right)^{-1} d \lambda\right] \\
G_{r, u, \infty} & =\frac{1}{2 \pi i} \psi_{u} \varphi \operatorname{Tr}_{s}\left[N_{Z} \int_{\delta} e^{-u^{2} \lambda}\left(\lambda-\mathcal{D}_{r, 1}^{2}\right)^{-1} d \lambda\right]
\end{aligned}
$$

Alors pour $2 \leq r \leq n$

$$
F_{r, u, \infty}+G_{r, u, \infty}=\varphi \operatorname{Tr}_{s}\left[N_{Z} \exp \left(-\mathcal{D}_{r, u}^{2}\right)\right] .
$$


On a l'analogue de la Proposition 1.10.

THÉORÈme 2.19. i) Il existe $\delta>0, C_{1}>0, C>0, T_{0}>0$ tels que pour $u \geq$ $1, T \geq T_{0}, 2 \leq r \leq n$,

$$
\left|F_{r, u, T}-F_{r, u, \infty}\right| \leq \frac{C}{T^{\delta}} e^{-C_{1} u}
$$

ii) Il existe des formes $a_{r, i, T}, b_{n, i, T}\left(T \in\left[T_{0},+\infty\right], 1 \leq r \leq n,-2 \operatorname{dim} S \leq i \leq 0\right)$, $\mathcal{C}^{\infty}$ sur $S$, telles que

$$
\begin{aligned}
& G_{r, u, \infty}=-\Sigma_{i=-2 \operatorname{dim} S}^{-1} a_{r, i, \infty} u^{i}+\operatorname{ch}^{\prime}\left(E_{r+1}, h^{E_{r+1}}\right), \\
& G_{n, u, T}=\Sigma_{i=-2 \operatorname{dim} S}^{-1} b_{n, i, T} u^{i}+\varphi \operatorname{Tr}_{s}\left[N_{Z} \exp \left(-\nabla_{T}^{H\left(Z, \xi_{\mid Z}\right), 2}\right)\right] .
\end{aligned}
$$

Quand $u \rightarrow 0$, uniformément en $T \geq T_{0}$, on a

$$
\begin{aligned}
& F_{r, u, T}=\Sigma_{i=-2 \operatorname{dim} S}^{0} a_{r, i, T} u^{i}+O(u) \text { pour } r \geq 2, \\
& G_{1, u, T}=-\Sigma_{i=-2 \operatorname{dim} S}^{0} a_{1, i, T} u^{i}+O(u) .
\end{aligned}
$$

iii) On $a$

$$
a_{r, 0, \infty}=\operatorname{ch}^{\prime}\left(E_{r}, h^{E_{r}}\right)-\operatorname{ch}^{\prime}\left(E_{r+1}, h^{E_{r+1}}\right), \quad \text { pour } \quad r \geq 2 .
$$

Il existe $\delta>0$ tel que pour $1 \leq r \leq n$, quand $T \rightarrow+\infty$, on a

$$
\begin{aligned}
& a_{r, i, T}=a_{r, i, \infty}+O\left(\frac{1}{T^{\delta}}\right), \\
& b_{n, i, T}=-a_{n, i, \infty}+O\left(\frac{1}{T^{\delta}}\right) \quad \text { pour } i<0, \\
& b_{n, i, T} T^{-(n-1) i}+\sum_{r=2}^{n} a_{r, i, T} T^{-(r-1) i}=-a_{1, i, T} \quad \text { pour } \quad i<0 .
\end{aligned}
$$

Preuve. i) En utilisant les Théorèmes 2.17 et 2.18 et en procédant comme en $[\mathrm{BL}, \S 11(\mathrm{p})$ et $\S 13(\mathrm{o})-(\mathrm{q})]$, on a $(2.98)$.

ii) Comme à l'intérieur de $\delta^{\prime}, 0$ est l'unique valeur propre possible des opérateurs $A_{n, 1, T}^{(0), 2}, D_{r}^{2}, D^{Y, 2}$, pour $r \geq 2, u \in \mathbf{C},|u| \geq 1$, on a

$$
\begin{aligned}
& G_{n, u, T}=\frac{1}{2 \pi i} \varphi \operatorname{Tr}_{s}\left[N_{3, T^{2(n-1)} u^{2}, T} \int_{\delta} e^{-\lambda}\left(\lambda-A_{n, u, T}^{2}\right)^{-1} d \lambda\right] \\
& G_{1, u, \infty}=\frac{1}{2 \pi i} \varphi \operatorname{Tr}_{s}\left[\left(N_{2, u^{2}}+N_{X}\right) \int_{\delta} e^{-\lambda}\left(\lambda-B_{2, u^{2}}^{2}\right)^{-1} d \lambda\right] \\
& G_{r, u, \infty}=\frac{1}{2 \pi i} \varphi \operatorname{Tr}_{s}\left[N_{Z} \int_{\delta} e^{-\lambda}\left(\lambda-\mathcal{D}_{r, u}^{2}\right)^{-1} d \lambda\right] .
\end{aligned}
$$

En utilisant (2.103) et en procédant comme en [B4, §9.13-9.14], on a (2.99).

iii) • Pour $T \in\left[T_{0},+\infty\right]$, soient

$$
f_{r, u, T}=\psi_{u}^{-1} F_{r, u, T}, \quad g_{r, u, T}=\psi_{u}^{-1} G_{r, u, T} .
$$

Alors $f_{r, u, T}$ et $g_{r, u, T}$ sont des fonctions holomorphes en $u \in \mathbf{C}$.

En utilisant les Théorèmes 2.14- 2.18, et en procédant comme en [BL, $\S 11$ (p) et $\S 13$ (o)-(q)], on sait qu'il existe aussi $C>0, \delta>0$ tels que pour tout $u \in \mathbf{C},|u| \leq 1$, $T \geq T_{0}$, on a

$$
\begin{gathered}
\left|f_{r, u, T}-f_{r, u, \infty}\right| \leq \frac{C}{T^{\delta}} \\
\left|g_{r, u, T}-g_{r, u, \infty}\right| \leq \frac{C}{T^{\delta}}
\end{gathered}
$$


En particulier, quand $T \rightarrow+\infty$, les fonctions $\left\{f_{r, u, T}\right\},\left\{g_{r, u, T}\right\}$ sont des fonctions holomorphes uniformément bornées en $\{u \in \mathbf{C},|u| \leq 1\}$. Elles ont donc un développement en série uniforme en $u$ au voisinage de $u=0$. De (2.105), et de la formule de Cauchy, on tire que les coefficients de ces développements convergent quand $T \rightarrow+\infty$ à la vitesse $O\left(\frac{1}{T^{\delta}}\right)$. De (2.104), on déduit le résultat correspondant pour les fonctions $F_{r, u, T}, G_{r, u, T}$ (qui sont elles-mêmes méromorphes en $u$ ). On a bien démontré (2.100) et les deux premières équations de (2.102).

- De (2.97), (2.99), (2.100), on tire (2.101).

- Comme en (1.64), par (2.38), pour $T \geq T_{0}$, on a

$$
G_{1, u, T}=\sum_{r=2}^{n} F_{r, T^{-r+1} u, T}+G_{n, T^{-(n-1)} u, T} .
$$

Par (2.99), (2.100) et (2.106), on a aussi la troisième équation de (2.102).

On a bien terminé la preuve du Théorème 2.19 . 1.6.

f) Preuve du Théorème 2.9. On procède comme dans la preuve du Théorème

En appliquant [Ma1, Théorème 5.15], on a

$$
\begin{aligned}
& \lim _{T \rightarrow+\infty} \int_{1}^{+\infty} 2\left\{\varphi \operatorname{Tr}_{s}\left[N_{3, u^{2}, T} \exp \left(-B_{3, u^{2}, T}^{2}\right)\right]-G_{1, u, T}\right\} \frac{d u}{u} \\
= & \int_{1}^{+\infty} 2\left\{\varphi \operatorname{Tr}_{s}\left[\left(N_{2, u^{2}}+N_{X}\right) \exp \left(-B_{2, u^{2}}^{2}\right)\right]-G_{1, u, \infty}\right\} \frac{d u}{u} .
\end{aligned}
$$

En utilisant le Théorème 2.19 et (2.106), pour $T \geq T_{0}$, on a

$$
\begin{aligned}
& \int_{1}^{+\infty} 2\left\{G_{1, u, T}-\varphi\right.\left.\operatorname{Tr}_{s}\left[N_{Z} \exp \left(-\nabla_{T}^{H\left(Z, \xi_{\mid}\right), 2}\right)\right]\right\} \frac{d u}{u}=\sum_{r=2}^{n} 2 \int_{T^{-(r-1)}}^{+\infty} F_{r, u, T} \frac{d u}{u} \\
&+2 \int_{T^{-(n-1)}}^{+\infty}\left\{G_{n, u, T}-\varphi \operatorname{Tr}_{s}\left[N_{Z} \exp \left(-\nabla_{T}^{H\left(Z, \xi_{\mid}\right), 2}\right)\right]\right\} \frac{d u}{u} .
\end{aligned}
$$

On décompose chaque intégrale $\int_{T^{-(r-1)}}^{+\infty}$ sous la forme $\int_{T^{-(r-1)}}^{1}+\int_{1}^{+\infty}$. En sommant les termes $\int_{1}^{+\infty}$, on obtient un terme $Q_{1, T}$, et en sommant les termes $\int_{T^{-(r-1)}}^{1}$, on obtient un terme $Q_{2, T}$. Alors par le Théorème 2.19 et le théorème de convergence dominée, quand $T \rightarrow+\infty$, on a

$$
\begin{aligned}
Q_{1, T} \rightarrow Q_{1, \infty}= & \sum_{r=2}^{n} 2 \int_{1}^{+\infty} F_{r, u, \infty} \frac{d u}{u} \\
& +2 \int_{1}^{+\infty}\left(G_{n, u, \infty}-\operatorname{ch}^{\prime}\left(E_{n+1}, h^{E_{n+1}}\right)\right) \frac{d u}{u} .
\end{aligned}
$$

En appliquant le Théorème 2.19, et le théorème de convergence dominée, quand $T \rightarrow+\infty$, comme en (1.71), on a

$$
\begin{aligned}
& Q_{2, T}-2 \sum_{r=2}^{n}(r-1)\left[\operatorname{ch}^{\prime}\left(E_{r}, h^{E_{r}}\right)-\operatorname{ch}^{\prime}\left(E_{r+1}, h^{E_{r+1}}\right)\right] \log T \\
& \rightarrow Q_{2, \infty}=\sum_{r=1}^{n-1} \int_{1}^{+\infty} 2\left(G_{r, u, \infty}-\operatorname{ch}^{\prime}\left(E_{r+1}, h^{E_{r+1}}\right)\right) \frac{d u}{u} \\
& \quad+2 \sum_{r=2}^{n} \int_{0}^{1}\left\{\varphi \operatorname{Tr}_{s}\left[N \exp \left(-\mathcal{D}_{r, u}^{2}\right)\right]-\operatorname{ch}^{\prime}\left(E_{r}, h^{E_{r}}\right)\right\} \frac{d u}{u}
\end{aligned}
$$


D'après (2.97), (2.107), (2.109) et (2.110), on a le Théorème 2.9.

g) Preuve du Théorème 2.10. On rappelle que $P_{T}$ est la projection orthogonale de $E_{0}$ sur $\operatorname{Ker} D_{T}^{Z}$ correspondant à $g_{T}^{T Z}, h^{\xi}$. Soit $\widetilde{P}_{T}$ la projection orthogonale de $E_{0} \operatorname{sur} \widetilde{E}_{T}=\operatorname{Ker} A_{T}^{(0)}$ associée à \langle\rangle$_{0}$.

D'après [BerB, (6.63)], pour $\alpha, \alpha^{\prime} \in E_{0}$, on a

$$
\left\langle P_{T} \alpha, P_{T} \alpha^{\prime}\right\rangle_{T}=\frac{1}{T^{2 \operatorname{dim} X}}\left\langle\widetilde{P}_{T} T^{N_{X}} B_{T} \alpha, \widetilde{P}_{T} T^{N_{X}} B_{T} \alpha^{\prime}\right\rangle_{0}
$$

Par (2.38) et (2.39), on a

$$
\widetilde{P}_{T}=\widetilde{p}_{n+1, T}
$$

En utilisant les Propositions 1.11 et 2.12 , et en procédant comme dans la preuve du Théorème 1.7, on a le Théorème 2.10 .

Remerciements. Cet article est une partie de ma thèse de doctorat de l'Université Paris XI (Orsay). Je tiens à exprimer ma plus profonde gratitude à mon directeur de thèse, le professeur J.M.Bismut. Il m'a soutenu tout au long de ce travail, du français aux mathématiques. Sans ses utiles discussions et suggestions, cet article n'aurait jamais pu été écrit. Je remercie aussi vivement le professeur Christophe Soulé pour ses observations, et le rapporteur pour ses remarques utiles.

\section{REFERENCES}

[BeGeV] N. Berline, E. Getzler, and M. Vergne, Heat Kernels and the Dirac Operator, Grundl. Math. Wiss. 298, Springer, Berlin, Heidelberg, New York, 1992.

[BerB] A. BERThOMIEU AND J.-M. Bismut, Quillen metric and higher analytic torsion forms, J. Reine Angew. Math, 457 (1994), pp. 85-184.

[B2] J.-M. BISmUT, Superconnection currents and complex immersions, Invent. Math., 99 (1990), pp. 59-113.

[B4] J.-M. BiSmUT, Families of Immersions, and Higher Analytic Torsion, Astérisque 244, 1997.

[BGS1] J.-M. BiSmut, H. Gillet, AND C. SoulÉ, Analytic torsion and holomorphic determinant bundles. I, Comm.Math. Phys., 115 (1988), pp. 49-78.

[BGS4] J.-M. Bismut, H. Gillet, AND C. SoulÉ, Bott-Chern currents and complex immerions, Duke Math. Journal, 60 (1990), pp. 255-284.

[BL] J.-M. Bismut And G. Lebeau, Complex Immersions and Quillen Metrics, Publ. Math. IHES. 74, 1991.

[BoC] R. BOTT AND S. S. CHERN, Hermitian vector bundles and the equidistribution of the zeross of their holomorphic sections, Acta Math., 114 (1968), pp. 71-112.

[CE] H. Cartan and S. Eilenberg, Homological Algebra, Princeton, 1956.

[D] X. DAI, Adiabatic limits, non multiplicativity of signature and Leray spectal sequence, J. A.M.S., 4 (1991), pp. 265-321.

[Go] R. Godement, Théorie des Faisceaux, Hermann, Paris, 1958.

[GrH] P. Griffiths And J. Harris, Principles of Algebraic Geometry, Wiley, New-York, 1978.

[Grot] A. GRothendieck, Sur quelques points d'algèbre homologique, Tôhoku Math. J., 9 (1957), pp. 119-221.

[H] R. HARTSHORNe, Algebraic Geometry, GTM 52, Springer, 1977.

[Ma] XIAONAN MA, Formes de torsion analytique et familles de submersions, C.R.A.S. Paris, série I, 324 (1997), pp. 205-210.

[Ma1] XiaOnAN MA, Formes de torsion analytique et familles de submersions I, Bull. Soc. Math. France, 127 (1999), pp. 541-621.

[M] B. Malgrange, Division des distributions, Séminaire Bourbaki 12 (1959/1960), $n^{\circ} 203$ (1960), p5.

[MM] R. MAzzeO AND R. MELRose, The adiabatic limit, Hodge cohomology and Leray's spectral sequence of a fibration, J. Diff. Geom., 31 (1990), pp. 185-213. 
